\title{
Side-channel Masking with Pseudo-Random Generator
}

\author{
Jean-Sébastien Coron ${ }^{1}$, Aurélien Greuet ${ }^{2}$, and Rina Zeitoun ${ }^{2}$ \\ 1 University of Luxembourg \\ jean-sebastien.coron@uni.lu \\ 2 IDEMIA, France \\ aurelien.greuet@idemia.com, rina.zeitoun@idemia.com
}

\begin{abstract}
High-order masking countermeasures against side-channel attacks usually require plenty of randomness during their execution. For security against $t$ probes, the classical ISW countermeasure requires $\mathcal{O}\left(t^{2} s\right)$ random bits, where $s$ is the circuit size. However running a True Random Number Generator (TRNG) can be costly in practice and become a bottleneck on embedded devices. In [IKL $\left.{ }^{+} 13\right]$ the authors introduced the notion of robust pseudo-random number generator (PRG), which must remain secure even against an adversary who can probe at most $t$ wires. They showed that when embedding a robust PRG within a private circuit, the number of random bits can be reduced to $\tilde{\mathcal{O}}\left(t^{4}\right)$, that is independent of the circuit size $s$ (up to a logarithmic factor). Using bipartite expander graphs, this can be further reduced to $\mathcal{O}\left(t^{3+\varepsilon}\right)$; however the resulting construction is impractical.

In this paper we describe a construction where the number of random bits is only $\tilde{\mathcal{O}}\left(t^{2}\right)$ for security against $t$ probes, without expander graphs; moreover the running time of each pseudo-random generation goes down from $\tilde{\mathcal{O}}\left(t^{4}\right)$ to $\tilde{\mathcal{O}}(t)$. Our technique consists in using multiple independent PRGs instead of a single one. We show that for ISW circuits, the robustness property of the PRG is not required anymore, which leads to simple and efficient constructions. For example, for AES we only need 48 bytes of randomness to get second-order security $(t=2)$, instead of 2880 in the original RivainProuff countermeasure. As a first feasibility result, we have implemented our countermeasure on an ARM-based embedded device with a relatively slow TRNG, and obtained a $50 \%$ speed-up compared to Rivain-Prouff.
\end{abstract}

\section{Introduction}

High-order masking. Side-channel analysis is a class of attacks which exploits the physical environment of a cryptosystem during its execution, to reveal the secrets being manipulated. The masking countermeasure is an efficient technique to protect sensitive data against this threat. To protect a sensitive data $x$, the masking technique consists in generating a random variable $r$ and manipulating the masked variable $x^{\prime}=x \oplus r$ and the random $r$ separately, instead of $x$ directly. In that case, every intermediate variable has the uniform distribution and any first-order attack is thwarted. However by combining information from both leakage points $x^{\prime}$ and $r$, a second-order attack can still be feasible (see for example [OMHT06]).

A natural countermeasure against high-order attacks is to use a high-order masking, where each variable $x$ is split into $n$ Boolean shares $x=x_{1} \oplus x_{2} \oplus \cdots \oplus x_{n}$, with $n>t$ for security against $t$ probes. Initially the shares are generated uniformly at random under this condition; for example one can generate $x_{1}, \ldots, x_{n-1}$ randomly and let $x_{n}=x \oplus x_{1} \oplus \cdots \oplus x_{n-1}$. The shares are then processed separately in masked operations (also called gadgets) that enable to compute the underlying secret variables in a secure way.

The study of circuits resistant against probing attacks was initiated by Ishai, Sahai and Wagner in [ISW03]. They showed how to transform any circuit of size $s$ into a circuit of size $\mathcal{O}\left(t^{2} s\right)$ secure against any adversary who can probe at most $t$ wires. The ISW construction is based on secret sharing every variable $x$ into $x=x_{1} \oplus x_{2} \oplus \cdots \oplus x_{n}$ as above, with $n=2 t+1$ shares to guarantee security against $t$ probes. Processing a XOR gate is straightforward as the shares can be xored separately. For processing an AND gate $z=x y$, one computes all cross-products $x_{i} y_{j}$ in Equation (1) below, and then uses a randomized algorithm to recombine the $n^{2}$ cross-products into an $n$-sharing of the output $z$.

$$
z=x y=\left(\bigoplus_{i=1}^{n} x_{i}\right) \cdot\left(\bigoplus_{i=1}^{n} y_{i}\right)=\underset{1 \leq i, j \leq n}{\bigoplus} x_{i} y_{j}
$$


Every AND gate is then expanded into a gadget of size $\mathcal{O}\left(t^{2}\right)$ and the resulting circuit has size $\mathcal{O}\left(t^{2} s\right)$.

The ISW construction was adapted to AES by Rivain and Prouff in [RP10], by working in $\mathbb{F}_{2^{8}}$ instead of $\mathbb{F}_{2}$. The authors observed that the non-linear part $S(x)=x^{254}$ of the AES SBox can be efficiently evaluated with only 4 non-linear multiplications over $\mathbb{F}_{2^{8}}$, and a few linear squarings. Each of those 4 multiplications can in turn be evaluated with the previous ISW gadget based on Equation (1), by working over $\mathbb{F}_{2^{8}}$ instead of $\mathbb{F}_{2}$.

Proving security. The approach initiated in [ISW03] for proving security against a $t$-probing adversary is based on simulation; one must show that the view of an adversary probing at most $t$ wires can be perfectly simulated without knowing the secret variables from the original circuit. To this aim, one shows that any set of $t$ probed variables can be perfectly simulated from the knowledge of at most $n-1$ input shares. Since any subset of $n-1$ input shares is uniformly and independently distributed, this ensures that the adversary learns nothing from the $t$ probes, since he could simulate them by himself. It was shown in [DDF14] that security against $t$ probes implies security against noisy leakage, under the assumption that every variable leaks independently.

Recently, the notions of (Strong) Non-Interference (NI/SNI) were introduced by Barthe et al. in $\left[\mathrm{BBD}^{+} 16\right]$, to allow easy composition of gadgets. The authors showed that the ISW multiplication gadget does satisfy the stronger $t$-SNI security definition. They also showed that with some additional mask refreshing, the Rivain-Prouff countermeasure for the full AES can be made secure with $n=t+1$ shares only, instead of $n=2 t+1$ shares in [ISW03].

More recently, a new security notion was introduced by Cassiers and Standaert in [CS18], called PINI, that allows even simpler composition of gadgets. Namely it suffices to ensure that all gadgets are PINI, and the composite gadget is then also PINI, which also implies security against $t$ probes. With its power and simplicity, the PINI definition appears to be the "right" notion for gadget security and composition; therefore we will use this definition in this paper, either by proving the PINI property of a gadget directly, or by first proving the $t$-SNI property and then PINI.

Minimizing randomness complexity. High-order masking countermeasures against side-channel attacks usually require plenty of randomness during their execution. The secure AND operation from [ISW03] with $t+1$ shares requires $t(t+1) / 2$ random bits, and therefore the randomness complexity of the ISW countermeasure is $\mathcal{O}\left(t^{2} s\right)$, where $s$ is the circuit size. More concretely, the evaluation of the AES SBox in Rivain-Prouff [RP10] requires the execution of 4 secure multiplications and 2 mask refreshing; each of those 6 gadgets requires $t(t+1) / 2$ fresh random bytes. For the 16 SBoxes and the 10 rounds of the AES, this amounts to generating $6 \times 16 \times 10 \times t(t+1) / 2=480 t(t+1)$ random bytes, which gives 2880 bytes for second-order security $(t=2)$.

However running a True Random Number Generator (TRNG) can be costly in practice and become a major bottleneck on embedded devices such as smart-cards. Thus, high-order resistant algorithms can rapidly become impractical when the number of shares grows. The main question is therefore how to minimize the number of TRNG calls while still guaranteeing $t$-probing security as in [ISW03].

Several attempts have been made to reduce the randomness complexity of private circuits. In $\left[\mathrm{BBP}^{+} 16\right]$, the authors showed a variant of the ISW multiplication with roughly $t^{2} / 4$ randoms instead of $t^{2} / 2$ in ISW. In [FPS17], the authors showed how to re-use randomness within several gadgets, thereby reducing the total amount of randomness needed, for small values of $t$ ( $t \leq$ 7). However the two above approaches only reduce the randomness complexity by a constant factor; that is, their asymptotic complexity is still $\mathcal{O}\left(t^{2} s\right)$ for circuit size $s$, as in the original ISW countermeasure.

A natural idea to reduce the number of calls to the TRNG is to use a pseudo-random generator (PRG) to generate all randoms in the circuit, while only a small seed will be generated by the TRNG. Obviously the PRG circuit should also be secure against probing attacks. We recall below that such approach, initiated by Ishai et al. in $\left[\mathrm{IKL}^{+} 13\right]$ with the concept of robust PRG, enables 
to reduce the randomness complexity of $t$-private circuits from $\mathcal{O}\left(t^{2} s\right)$ to $\mathcal{O}\left(t^{4}(\log s+\log t)\right)$; with respect to the circuit size $s$, this is therefore an exponential improvement. Our main contribution is this paper will be to reduce this complexity further down to $\mathcal{O}\left(t^{2}(\log s+\log t)\right)$, and to describe a concrete implementation of AES based on this approach. We refer to Table 2 below for the number of bytes required to protect AES against $t$-th order attacks; we see that for small values of $t$, we obtain almost two orders of magnitude improvement compared to previous methods.

Robust PRGs and private circuits. In $\left[\mathrm{IKL}^{+} 13\right]$, the authors introduced the notion of robust pseudo-random number generator (PRG). A robust PRG must remain secure even if an adversary can probe at most $t$ intermediate variables in the PRG circuit. The authors showed that such robust PRG can be used in the ISW countermeasure to minimize the randomness complexity. Namely the resulting circuit uses a short random seed only, and remains secure against $t$-th order attacks.

Recall that the original ISW countermeasure requires $\mathcal{O}\left(t^{2} s\right)$ bits of randomness, where $s$ is the circuit size. Following [ $\left.\mathrm{IKL}^{+} 13\right]$, we first recall how this can be reduced to $\mathcal{O}\left(t^{4}(\log t+\log s)\right)$, using a trivial construction of robust PRG. More precisely, the construction is based on $r$-wise independent PRG. A PRG is said to be $r$-wise independent if any subset of at most $r$ output bits of the PRG is uniformly and independently distributed. The authors show that the ISW countermeasure can be adapted so that any wire in the ISW circuit depends on at most $\ell=\mathcal{O}\left(t^{2}\right)$ bits of randomness; such parameter $\ell$ is called the locality of the randomness and will play a crucial role in this paper. Since the adversary can probe at most $t$ wires, the adversary's side-channel observation can then depend on at most $t \cdot \ell=\mathcal{O}\left(t^{3}\right)$ bits of randomness. Therefore, instead of using a TRNG, it is sufficient to use an $r$-wise independent PRG with parameter $r=t \cdot \ell=\mathcal{O}\left(t^{3}\right)$; if the $r$-wise PRG is secure against $t$ probes, as shown in $\left[\mathrm{IKL}^{+} 13\right]$ the resulting circuit will remain secure against $t$ probes.

It is easy to obtain an $r$-wise independent PRG by evaluating a degree $r-1$ polynomial on distinct inputs in a finite field $\mathbb{F}$; the $r$ coefficients of the polynomials are initially generated at random in $\mathbb{F}$; this is the seed of the PRG. From $r$ fresh randoms in $\mathbb{F}$, one can then obtain $m$ pseudo-randoms with the $r$-wise independence property, as long as $m \leq|\mathbb{F}|$. To obtain an $r$-wise independent PRG with robustness against $t$ probes, as observed in $\left[\mathrm{IKL}^{+} 13\right]$ a trivial construction consists in xoring the output of $t+1$ PRGs, so that at least one PRG has not been probed. One can therefore obtain an $r$-wise independent PRG robust against $t$ probes by using $r \cdot(t+1)=\mathcal{O}\left(t^{4}\right)$ fresh randoms in $\mathbb{F}$ as input, and such PRG can then generate $m \leq|\mathbb{F}|$ pseudo-randoms in $\mathbb{F}$. Since the original ISW countermeasure requires $m=\mathcal{O}\left(t^{2} s\right)$ randoms (where $s$ is the circuit size), using $\mathbb{F}=\mathbb{F}_{2^{k}}$ one can take $k=\mathcal{O}(\log m)=\mathcal{O}(\log t+\log s)$. One therefore needs $\mathcal{O}\left(t^{4}(\log t+\log s)\right)=$ $\tilde{\mathcal{O}}\left(t^{4}\right)$ bits of randomness ${ }^{1}$, instead of $\mathcal{O}\left(t^{2} s\right)$. The number of input random bits is then independent of the circuit size $s$ (up to some logarithmic factor). In summary, any $t$-private circuit in which each wire depends on at most $\ell$ bits of randomness can be converted into a $t$-private circuit using roughly $t^{2} \ell$ bits of randomness via the use of robust $r$-wise PRGs. As written by the authors: "Improving the randomness locality $\ell$ of private circuits would immediately yield a corresponding improvement [in the number of input random bits].".

In $\left[\mathrm{IKL}^{+} 13\right]$, the authors describe an improved construction of robust PRG, based on unbalanced bipartite expander graphs. Using the Guruswami-Umans-Vadhan construction of expander graphs [GUV09], they obtain $r$-wise independence and resistance against $t=r$ probes with $r^{1+\eta}$ bits of true randomness as input, for any $\eta>0$. In the context of the ISW countermeasure, this enables to use $\mathcal{O}\left(t^{3+\varepsilon}\right)$ random bits as input for any $\varepsilon>0$, instead $\tilde{\mathcal{O}}\left(t^{4}\right)$. In Appendix A we provide a simplified proof of strong robustness for expander graph based PRG, based on the proof of weak robustness from $\left[\mathrm{IKL}^{+} 13\right]$. We also argue that for minimizing the amount of input randomness, expander graph based constructions are actually impractical.

\footnotetext{
${ }^{1}$ We use the notation $f(\lambda)=\tilde{\mathcal{O}}(g(\lambda))$ if $f(\lambda)=\mathcal{O}\left(g(\lambda) \log ^{k} \lambda\right)$ for some $k \in \mathbb{N}$. For simplicity we assume that the circuit size $s$ is polynomially bounded in $t$.
} 
Our contribution. Our main contribution is a countermeasure against side-channel attacks where the number of random bits is only $\tilde{\mathcal{O}}\left(t^{2}\right)$ for security against $t$ probes, independently of the circuit size (up to a logarithmic factor), and without using expander graphs. Moreover the running time of pseudo-random generation goes down from $\tilde{\mathcal{O}}\left(t^{4}\right)$ to $\tilde{\mathcal{O}}(t)$. We summarize in Table 1 below the asymptotic complexities of existing techniques and our new techniques. We proceed in two steps.

In the first step, we show how to improve the locality $\ell$ of private circuits from $\ell=\mathcal{O}\left(t^{2}\right)$ down to $\ell=\mathcal{O}(t)$. As illustrated in the third line of Table 1 below, reducing $\ell$ from $\mathcal{O}\left(t^{2}\right)$ to $\mathcal{O}(t)$ enables to reduce the $r$-wise independence parameter from $r=\mathcal{O}\left(t^{3}\right)$ down to $r=\mathcal{O}\left(t^{2}\right)$; the number of input random bits is then now decreased from $\tilde{\mathcal{O}}\left(t^{4}\right)$ to $\tilde{\mathcal{O}}\left(t^{3}\right)$ with the trivial construction (and from $\mathcal{O}\left(t^{3+\varepsilon}\right)$ to $\mathcal{O}\left(t^{2+\varepsilon}\right)$ with expander graphs). Our technique is as follows. The authors of [IKL $\left.{ }^{+} 13\right]$ obtain $\ell=\mathcal{O}\left(t^{2}\right)$ by performing a mask locality refreshing at the end of each ISW multiplication gadget. Instead we modify the ISW multiplication by performing a series of internal locality refreshing. For this we consider successive $i \times i$ ISW submatrices and perform a mask refreshing after the processing of each submatrix; these internal mask refreshing enable to bring the locality down to $\ell=\mathcal{O}(t)$. We have also performed a formal verification of our new algorithms, using the CheckMasks tool [Cor18], for both the locality and the security properties; we provide the source code in [Cor19a]. This first step is described in Section 3.

In the second step, our technique consists in using multiple independent PRGs instead of a single one. This has two main advantages. The first advantage is that for ISW circuits, one can show that the robustness property of the PRG is not required anymore; this implies that we can use a very simple PRG based on polynomial evaluation as above. The second advantage is that the locality with respect to each subset of randoms generated by each PRG becomes $\ell=\mathcal{O}(1)$. Therefore each independent PRG can be $r$-wise independent with a much smaller parameter $r=\mathcal{O}(t)$ instead of $r=\mathcal{O}\left(t^{3}\right)$, and therefore requires only $r=\mathcal{O}(t)$ randoms in the finite field (since robustness is not needed). In that case, we need $\mathcal{O}\left(t^{2}\right)$ independent PRGs and therefore the size of the input randomness is $\tilde{\mathcal{O}}\left(t^{3}\right)$; see Line 4 of Table 1 . Finally, when using internal locality refreshing as in the first step above, we only need $\mathcal{O}(t)$ independent PRGs, and eventually the number of input random bits is reduced to $\tilde{\mathcal{O}}\left(t^{2}\right)$, instead of $\mathcal{O}\left(t^{3+\varepsilon}\right)$ with expander graphs in [IKL $\left.{ }^{+} 13\right]$ (see Line 5 of Table 1$)$. We stress that this asymptotic improvement over $\left[\mathrm{IKL}^{+} 13\right]$ is obtained without using expander graphs, that is we can use a simple PRG based on polynomial evaluation in a finite field (see Section 4). ${ }^{2}$

As mentioned previously, we found that expander graphs PRG are impractical for minimizing the amount of input randomness. However expander graphs can still be useful for optimizing the time generation of each pseudo-random; namely the output locality of an expander graph PRG (i.e., the number of inputs on which each output depends) can be at most polylogarithmic in the seed length (as opposed to linear for a PRG based on polynomial evaluation); hence in Table 1 the pseudo-random time generation is always $\tilde{\mathcal{O}}(1)$. In Section 2.3 we give an example of a simple construction based on expander graph that achieves very fast pseudo-random generation, at the cost of significantly more input randomness.

Finally, we describe in Section 5 an application of our countermeasure to AES. We show that for AES we only need 48 bytes of randomness to get second-order security $(t=2)$, instead of 2880 in the original Rivain-Prouff countermeasure. We see in Table 2 below that for small values of $t$, our construction reduces the randomness complexity of masking AES by almost 2 orders of magnitude. In Section 5, we also provide the results of a concrete implementation. When implemented on an ARM-based embedded device with a relatively slow TRNG, we obtain a 50\% speed-up compared to Rivain-Prouff for $t=2$. We provide the source code in $\mathrm{C}$ in [Cor19b]. Needless to say, we do not claim that in practice our implementation would be secure against a $t$-th order attack. Namely the implementation is only provided for illustrative purpose, and timing comparisons. Obtaining a secure implementation would require to (at least) carefully examine the assembly code, and perform a leakage test with concrete acquisitions from an oscilloscope.

${ }^{2}$ The proceedings version of [AIS18] claimed to achieve randomness complexity $\mathcal{O}\left(t^{1+\varepsilon}\right)$, but the claim was later retracted in the final version. 


\begin{tabular}{|c|c|c|c|c|c|c|}
\hline & \#PRG & loc. $\ell$ & $r$-wise & PRG & TRNG & time PRG \\
\hline ISW without PRG [ISW03] & - & - & - & - & $\mathcal{O}\left(t^{2} s\right)$ & - \\
\hline \multirow{2}{*}{ ISW with Final LR, single PRG $\left[\mathrm{IKL}^{+} 13\right]$} & \multirow{2}{*}{1} & \multirow{2}{*}{$\mathcal{O}\left(t^{2}\right)$} & \multirow{2}{*}{$\mathcal{O}\left(t^{3}\right)$} & Trivial & $\tilde{\mathcal{O}}\left(t^{4}\right)$ & $\tilde{\mathcal{O}}\left(t^{4}\right)$ \\
\hline & & & & EG & $\mathcal{O}\left(t^{3+\varepsilon}\right)$ & $\tilde{\mathcal{O}}(1)$ \\
\hline \multirow{2}{*}{ ISW with Internal LR, single PRG (Sec. 3) } & \multirow{2}{*}{1} & \multirow{2}{*}{$\mathcal{O}(t)$} & \multirow{2}{*}{$\mathcal{O}\left(t^{2}\right)$} & Trivial & $\tilde{\mathcal{O}}\left(t^{3}\right)$ & $\tilde{\mathcal{O}}\left(t^{3}\right)$ \\
\hline & & & & EG & $\mathcal{O}\left(t^{2+\varepsilon}\right)$ & $\tilde{\mathcal{O}}(1)$ \\
\hline \multirow{2}{*}{ ISW with Final LR, multiple PRGs (Sec. 4) } & \multirow{2}{*}{$\mathcal{O}\left(t^{2}\right)$} & \multirow{2}{*}{$\mathcal{O}(1)$} & \multirow{2}{*}{$\mathcal{O}(t)$} & Linear & $\tilde{\mathcal{O}}\left(t^{3}\right)$ & $\tilde{\mathcal{O}}(t)$ \\
\hline & & & & EG & $\mathcal{O}\left(t^{3+\varepsilon}\right)$ & $\tilde{\mathcal{O}}(1)$ \\
\hline \multirow{2}{*}{ ISW with Internal LR, multiple PRGs (Sec. 4) } & \multirow{2}{*}{$\mathcal{O}(t)$} & \multirow{2}{*}{$\mathcal{O}(1)$} & \multirow{2}{*}{$\mathcal{O}(t)$} & Linear & $\tilde{\mathcal{O}}\left(t^{2}\right)$ & $\tilde{\mathcal{\mathcal { O }}}(t)$ \\
\hline & & & & EG & $\mathcal{O}\left(t^{2+\epsilon}\right)$ & $\tilde{\mathcal{O}}(1)$ \\
\hline
\end{tabular}

Table 1. Asymptotic efficiency of various constructions. The Locality Refreshing (LR) is performed either at the end of each gadget (Line 2 and Line 4), or sequentially within each gadget (Line 3 and Line 5). The trivial construction of PRG is based on xoring $t+1$ linear PRGs to get robustness against $t$ probes.

\begin{tabular}{|l|c|c|c|c|c|c|}
\cline { 2 - 7 } \multicolumn{1}{c|}{} & $t=2$ & $t=3$ & $t=4$ & $t=5$ & $t=6$ & $t=7$ \\
\hline Rivain-Prouff [RP10] & 2880 & 5760 & 9600 & 14400 & 20160 & 26880 \\
\hline Belaïd et. al [BBP $\left.{ }^{+} 16\right]$ & 2560 & 5120 & 8000 & 13120 & 18240 & 24000 \\
\hline Faust et. al [FPS17] & 1415 & 2530 & 6082 & 6699 & 20712 & 20726 \\
\hline This paper & 48 & 108 & 192 & 300 & 432 & 588 \\
\hline
\end{tabular}

Table 2. Number of bytes of randomness to get $t$-th order security for AES.

\section{Definitions and Previous Work}

\subsection{Private circuits}

In 2003, Ishai, Sahai and Wagner [ISW03] initiated the study of securing circuits against an attacker who can probe a fraction of its wires. They showed how to transform any circuit of size $|C|$ into a larger circuit of size $O\left(|C| \cdot t^{2}\right)$ with the same functionality but secure against a $t$-probing adversary, based on splitting each variable $x$ into $n=2 t+1$ shares with $x=x_{1} \oplus x_{2} \oplus \cdots \oplus x_{n}$.

Definition 1 (Private circuit). A private circuit for $f:\{0,1\}^{n_{i}} \rightarrow\{0,1\}^{n_{o}}$ is a triple $(I, C, O)$ where $I:\{0,1\}^{n_{i}} \rightarrow\{0,1\}^{\hat{n}_{i}}$ is a randomized input encoder, $C$ is a randomized boolean circuit with input $\hat{\omega} \in\{0,1\}^{\hat{n}_{i}}$, output $\hat{y} \in\{0,1\}^{\hat{n}_{o}}$, and randomness $\rho \in\{0,1\}^{m}$, and $O:\{0,1\}^{\hat{n}_{o}} \rightarrow\{0,1\}^{n_{o}}$ is an output decoder, such that for any input $\omega \in\{0,1\}^{n_{i}}$ we have $\operatorname{Pr}[O(C(I(\omega), \rho))=f(\omega)]=1$, where the probability is over the randomness of $I$ and $\rho$.

For $I$ and $O$ we consider the canonical encoder and decoder: $I$ encodes each input bit $\omega_{i}$ by a vector of $2 t+1$ random bits with parity $\omega_{i}$, and $O$ takes the parity of each block of $2 t+1$ bits.

Definition 2 ( $t$-privacy). We say that $C$ is a $t$-private implementation of $f$ with encoder $I$ and decoder $O$ is t-private (or t-probing secure) if for any $\omega, \omega^{\prime} \in\{0,1\}^{n_{i}}$ and any set $P$ of $t$ wires in $C$, the distributions $C_{P}(I(\omega), \rho)$ and $C_{P}\left(I\left(\omega^{\prime}\right), \rho\right)$ are identical, where $C_{P}$ denotes the set of $t$ values on the wires from $P$.

\section{$2.2 \quad$ PINI and $t$-SNI security}

The Probe Isolating Non-Interference (PINI) security notion was introduced in [CS18] to enable easy composition of gadgets. Let $n$ be the number of shares. We let $x_{\star}=\left(x_{i}\right)_{i=1, \ldots n}$ be an $n$-sharing of $x$ if $x=\bigoplus_{i=1}^{n} x_{i}$. Given a subset $I \subset[1, n]$ of share indices, we denote by $x_{\mid I}:=\left\{x_{i}: i \in I\right\}$ the corresponding subset of shares. A gadget with $m$ inputs and $\ell$ outputs is a circuit with $m n$ input shares grouped into $m n$-sharings denoted $\left(x_{\star, 1}, \ldots x_{\star, m}\right)$, and similarly $\ell n$ output shares denoted 
$\left(y_{\star, 1}, \ldots y_{\star, \ell}\right)$. For a given share index $i$, we also use the notation $x_{i, \star}=\left\{x_{i, j}: 1 \leq j \leq m\right\}$ to denote all shares with index $1 \leq i \leq n$; similarly, we also write $x_{\mid I, \star}=\left\{x_{i, \star}: i \in I\right\}$. Below we recall the Probe Isolating Non-Interference (PINI) definition from [CS18]; we actually use a slightly simplified (and equivalent) definition compared to [CS18]; we explain the difference in Appendix B.1.

Definition 3 (PINI [CS18] (adapted)). Let $G$ be a gadget with input shares $x_{i, \star}$ and output shares $y_{i, \star}$ for $1 \leq i \leq n$. The gadget $G$ is PINI if for any $t_{1} \in \mathbb{N}$, any set of $t_{1}$ intermediate variables and any subset $\mathcal{O}$ of output indices, there exists a subset $I \subset[1, n]$ of input indices with $|I| \leq t_{1}$ such that the $t_{1}$ intermediate variables and the output shares $y_{\mid \mathcal{O}, \star}$ can be perfectly simulated from the input shares $x_{\mid I \cup \mathcal{O}, \star}$.

It is straightforward to show that a PINI gadget with $n$ shares is secure against $t=n-1$ probes. We recall the proof of PINI composition (under our slightly modified definition) in Appendix B.1.

Proposition 1 (PINI security [CS18]). Any PINI gadget with $n$ shares is $(n-1)$-probing secure.

Proposition 2 (PINI composition [CS18]). Any composite gadget made of PINI composing gadgets is PINI.

Below we recall the SNI security notion introduced in $\left[\mathrm{BBD}^{+} 15\right]$. We consider a gadget taking as input two $n$-tuples $\left(x_{i}\right)_{1 \leq i \leq n}$ and $\left(y_{i}\right)_{1 \leq i \leq n}$ of shares, and outputting a single $n$-tuple $\left(z_{i}\right)_{1 \leq i \leq n}$. As previously, given a subset $I \subset[1, n]$, we denote by $x_{\mid I}$ all elements $x_{i}$ such that $i \in I$.

Definition 4 ( $t$-SNI security). Let $G$ be a gadget taking as input $n$ shares $\left(x_{i}\right)_{1 \leq i \leq n}$ and $n$ shares $\left(y_{i}\right)_{1 \leq i \leq n}$, and outputting $n$ shares $\left(z_{i}\right)_{1 \leq i \leq n}$. The gadget $G$ is said to be $t$-SNI secure if for any set of $t_{1}$ probed intermediate variables and any subset $\mathcal{O}$ of output indices, such that $t_{1}+|\mathcal{O}| \leq t$, there exist two subsets $I$ and $J$ of input indices which satisfy $|I| \leq t_{1}$ and $|J| \leq t_{1}$, such that the $t_{1}$ intermediate variables and the output variables $z_{\mid \mathcal{O}}$ can be perfectly simulated from $x_{\mid I}$ and $y_{\mid J}$

Intuitively, the $t$-SNI security definition provides an "isolation" between the output shares and the input shares, so that the number of input variables required for the simulation is upper-bounded by the number of internal probes $t_{1}$, and does not depend on the number of output variables that must be simulated, as long as $t_{1}+|\mathcal{O}| \leq t$. There is an analogous definition for a gadget with a single input $\left(x_{i}\right)_{1 \leq i \leq n}$; in that case, the simulation is performed from $x_{\mid I}$ with $|I| \leq t_{1}$.

It is easy to see that for a single input gadget, $(n-1)$-SNI security implies PINI security. Moreover, for a 2-input ( $n-1)$-SNI gadget as considered in Definition 4, as shown in [CS18] we can obtain a PINI gadget by pre-refreshing one of the inputs with a $(n-1)$-SNI mask refreshing algorithm; this is the double-SNI approach (see Fig. 1). A mask refreshing gadget takes as input the $n$-sharing of a value $x$ and outputs a randomized $n$-sharing of the same value $x$. Therefore, in this paper, our strategy for proving gadget security is either to directly prove the PINI property, or to first prove the $t$-SNI property and then apply the "double-SNI" strategy. Note that for specific circuits such as the AES SBox, one can use some optimization; for example the full SBox computation can be proven $t$-SNI and therefore PINI with 4 multiplications and 2 mask refreshing only (instead of 4 mask refreshing as in the naive "double-SNI" strategy).

Proposition 3 (Double-SNI [CS18]). Let $G$ be a $(n-1)$-SNI gadget taking as input $\left(a_{i}\right)_{1 \leq i \leq n}$ and $\left(b_{i}\right)_{1 \leq i \leq n}$, and outputting $\left(c_{i}\right)_{1 \leq i \leq n}$. Let $R$ be a $(n-1)$-SNI gadget taking as input $\left(x_{i}\right)_{1 \leq i \leq n}$ and outputting $\left(y_{i}\right)_{1 \leq i \leq n}$. The composite gadget $G^{\prime}$ taking as input $\left(x_{i}\right)_{1 \leq i \leq n}$ and $\left(b_{i}\right)_{1 \leq i \leq n}$, and outputting $\left(c_{i}\right)_{1 \leq i \leq n}$, with $G^{\prime}\left(\left(x_{i}\right),\left(b_{i}\right)\right)=G\left(R\left(\left(x_{i}\right)\right),\left(b_{i}\right)\right)$ is PINI.

Finally, we recall in Appendix B.2 the SecMult gadget used in [RP10] for protecting AES against $t$-th order attacks. It is an extension to $\mathbb{F}_{2^{k}}$ of the original ISW countermeasure [ISW03] described in $\mathbb{F}_{2}$. The SecMult gadget was proven $t$-SNI in $\left[\mathrm{BBD}^{+} 16\right]$. We also recall in Appendix 


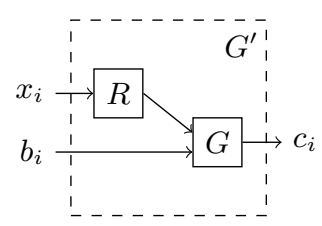

Fig. 1. The double-SNI approach: when both gadgets $G$ and $R$ are $(n-1)$-SNI, the composite gadget $G^{\prime}$ is PINI.

B.2 the mask refreshing gadget FullRefresh introduced by Duc et. al in [DDF14], based on SecMult; it was also proven $t$-SNI in $\left[\mathrm{BBD}^{+} 16\right]$. We can therefore use the FullRefresh gadget to apply the above "double-SNI" strategy. Moreover, in this paper, when we describe a variant of SecMult, we apply the same modifications to the FullRefresh gadget; this is straightforward, since the FullRefresh gadget can be seen as a SecMult with one input equal to $(1,0, \ldots, 0)$.

\section{3 r-wise independent PRG: definition and construction}

We recall the definition of an $r$-wise independent pseudo-random generator (PRG). We denote by $U_{n}$ the uniform distribution in $\{0,1\}^{n}$.

Definition 5 ( $r$-wise independent PRG). A function $G:\{0,1\}^{n} \rightarrow\{0,1\}^{m}$ is an $r$-wise independent pseudo-random generator if any subset of $r$ bits of $G(x)$ is uniformly and independently distributed when $x \leftarrow U_{n}$

We can construct an $r$-wise independent PRG via polynomial evaluation in a finite field $\mathbb{F}$. Letting $\boldsymbol{a}=\left(a_{0}, \ldots, a_{r-1}\right) \in \mathbb{F}^{r}$, we consider the polynomial:

$$
h_{\boldsymbol{a}}(x)=\sum_{i=0}^{r-1} a_{i} x^{i}
$$

For any $m \leq|\mathbb{F}|$, we can define the function $G: \mathbb{F}^{r} \rightarrow \mathbb{F}^{m}$ by letting:

$$
G(\boldsymbol{a})=\left(h_{\boldsymbol{a}}(0), \ldots, h_{\boldsymbol{a}}(m-1)\right)
$$

where we assume that we have some indexing of the field elements in $\mathbb{F}$. The function $G$ is an $r$-wise independent PRG because there is a bijection between the $r$ coefficients of a polynomial of degree at most $r-1$ and its evaluation at $r$ distinct points $x_{i}$.

For $\mathbb{F}=\mathbb{F}_{2^{k}}$, this gives an $r$-wise independent PRG taking as input $r k$ bits and outputting at most $k \cdot 2^{k}$ bits. Namely when working over $\mathbb{F}_{2^{k}}$ and generating $k$-bit pseudo-randoms, we can use each individual bit of the $k$-bit pseudo-random, and the PRG function remains $r$-wise independent. The parameter $k$ determines the expansion factor of the PRG. For our application to AES in Section 5, for simplicity we will work over $\mathbb{F}_{2^{16}}$, using $\mathbb{F}_{2^{8}}$ as a subfield. For a block-cipher using single bits, one would work in $\mathbb{F}_{2^{k}}$ and use each of the $k$ bits of $\mathbb{F}_{2^{k}}$ separately.

A simple 3-wise independent PRG. We also consider a very simple PRG that achieves 3-wise independence only. We consider a set of $2 d$ random bits $x_{i}$ and $y_{i}$ for $1 \leq i \leq d$. We define the following function $G:\{0,1\}^{2 d} \rightarrow\{0,1\}^{d^{2}}$ :

$$
G\left(x_{1}, \ldots, x_{d}, y_{1}, \ldots, y_{d}\right)=\left(x_{i} \oplus y_{j}\right)_{1 \leq i, j \leq d}
$$

The function $G$ can be seen as a PRG based on expander graph; see Fig. 9 in Appendix A.

Lemma 1. The function $G$ is a 3-wise independent $P R G$. 
Proof. We must show that any 3 variables $\left(x_{i_{1}} \oplus y_{j_{1}}\right),\left(x_{i_{2}} \oplus y_{j_{2}}\right)$ and $\left(x_{i_{3}} \oplus y_{j_{3}}\right)$ are uniformly and independently distributed.

We distinguish 3 cases. If $\#\left\{i_{1}, i_{2}, i_{3}\right\}=3$, then the three values are independent thanks to randoms $x_{i_{1}}, x_{i_{2}}$ and $x_{i_{3}}$. If $i_{1}=i_{2}=i_{3}$, then we must have $\#\left\{j_{1}, j_{2}, j_{3}\right\}=3$ and the three values are independent thanks to randoms $y_{j_{1}}, y_{j_{2}}$ and $y_{j_{3}}$. Eventually, if exactly two indices among $i_{1}$, $i_{2}$ and $i_{3}$ are equal, say wlog $i_{1}=i_{2} \neq i_{3}$, then we must have $j_{1} \neq j_{2}$ and the randoms $y_{j_{1}}, y_{j_{2}}$ and $x_{i_{3}}$ ensure the independence of the three values.

\subsection{Robust PRG: definition and trivial construction}

In $\left[\mathrm{IKL}^{+} 13\right]$, the authors introduced the notion of robust pseudo-random number generator (PRG), which should remain secure even if an adversary can probe at most $k$ intermediate variables in the PRG circuit. We recall the definition of (strongly) robust PRG from [IKL $\left.{ }^{+} 13\right]$ below. Under this definition, the output bits of the PRG must remain $r$-wise independent outside some set $T$ of bounded size, conditioned on the values of any set $S$ of at most $k$ probes in the PRG circuit and the outputs in $T$. In $\left[\mathrm{IKL}^{+} 13\right]$ the authors actually consider the robustness of three distinct types of PRG: $r$-wise independent PRGs, where as above any $r$ bits of output must be uniformly and independently distributed, small-bias PRGs, where the distinguisher can compute the parity of any subset of the outputs, and cryptographic PRGs, where the distinguisher is limited to arbitrary polynomial-time computations. In the following we only consider $r$-wise independent PRGs.

In this paper we actually use a slightly weaker definition of strong robustness compared to $\left[\mathrm{IKL}^{+} 13\right]$, in which we allow the output bits outside the set $T$ to be only $(r-q|S|)$-wise independent, instead of $r$-wise independent, where $|S| \leq k$ is the number of probes and $q$ a parameter. In other words, we allow the $r$-wise independence of the PRG to degrade gracefully with the number of probes. This will give slightly more efficient constructions; in particular, the trivial construction of xoring $k+1$ PRGs will only require the $r$-wise independence of each PRG, instead of the $(r+k)$-wise independence in $\left[\mathrm{IKL}^{+} 13\right]$. Obviously we need to ensure that a robust PRG under our definition can still be embedded in a private circuit with the same parameters as in $\left[\mathrm{IKL}^{+} 13\right]$; see Theorem 1 below.

Definition 6 (Strong robust PRG $\left[\mathbf{I K L}^{+} \mathbf{1 3}\right]$ (adapted)). A circuit implementation $C$ of a PRG $G:\{0,1\}^{n} \rightarrow\{0,1\}^{m}$ is strong $(r, k, q)$-robust if given $Y=G(X)$ where $X \leftarrow\{0,1\}^{n}$, for any set $S$ of at most $k$ probes in $C$, there is a set $T$ of at most $q|S|$ output bits such that conditioned on any fixing of the values $C_{S}$ of the wires in $S$ and of $Y_{T}$, the values $Y_{\bar{T}}$ of the output bits not in $T$ are $(r-q|S|)$-wise independent and uniformly distributed.

Trivial construction. As noted in $\left[\mathrm{IKL}^{+} 13\right]$, we can obtain a strong $(r, k, 1)$-robust PRG by taking the xor of $k+1$ PRGs, each with the $r$-wise independence property. More precisely, letting $g:\{0,1\}^{n} \rightarrow\{0,1\}^{m}$, we let $G:\{0,1\}^{n \cdot(k+1)} \rightarrow\{0,1\}^{m}:$

$$
G\left(x_{1}, \ldots, x_{k+1}\right)=g\left(x_{1}\right) \oplus g\left(x_{2}\right) \oplus \cdots \oplus g\left(x_{k+1}\right)
$$

where the xors are performed from left to right.

Lemma 2 (Strong robustness of $G$ ). If $g$ is an $r$-wise independent $P R G$, then $G$ is a strong $(r, k, 1)$-robust $P R G$.

Proof. Since there are at most $k$ probes and $k+1$ PRGs, there exists an index $i^{\star}$ such that $g\left(x_{i^{\star}}\right)$ has not been probed. In the following, we fix all inputs $x_{i}$ except $x_{i^{\star}}$.

Let $t \leq k$ be the number of probes. We consider the set $T$ of indices $j \in[1, m]$ such that the $j$-th bit of any partial sum $g\left(x_{1}\right) \oplus \cdots \oplus g\left(x_{i}\right)$ is probed. We must have $|T| \leq t$. Since $g$ is an $r$-wise independent PRG, by definition any set of $r$ output bits of $g\left(x_{i}^{\star}\right)$ is uniformly and independently distributed; this implies that any set of $r-t$ output bits of $g\left(x_{i}^{\star}\right)$ with indices outside $T$ are uniformly and independently distributed, even conditioned on the output bits in $T$ and the other probes. Since we have fixed the inputs of all other PRGs, this also applies for the output of $G$. Therefore $G$ is a strong $(r, k, 1)$-robust PRG. 
Expander graph construction. Using an explicit construction of a bipartite expander graph [GUV09], the authors of [IKL $\left.{ }^{+} 13\right]$ obtain a construction of a strong $(r, k, q)$-robust PRG with $r, k=n^{1-\eta}$ where $n$ is the number of random input bits, for any $\eta>0$. In Appendix A we provide a simplified proof of strong robustness for expander graph based PRG, based on the proof of weak robustness from $\left[\mathrm{IKL}^{+} 13\right]$. We also argue that for minimizing the amount of input randomness, while asymptotically better than the trivial construction, expander graph based constructions are actually impractical. Namely in our analysis the expander graph PRG construction based on [GUV09] becomes better than the trivial construction only for $r \geq 2^{18}$ and at least $2^{36}$ random input bits.

\subsection{Application to private circuits}

We recall below the main theorem from $\left[\mathrm{IKL}^{+} 13\right]$, showing that we can plug a robust PRG in a private circuit to generate all randomness from a small random seed, and the resulting construction remains secure against probing attacks. Firstly an important parameter is the locality $\ell$ of the randomness in the circuit.

Definition 7 (Randomness locality $\left[\mathbf{I K L}^{+} \mathbf{1 3}\right]$ ). A circuit $C$ is said to make an $\ell$-local use of its randomness if the value of each of its wires is determined by its (original, unmasked) input and at most $\ell$ bits of the randomness used in the circuit.

Theorem 1 (Private circuit with PRG [IKL $\left.{ }^{+} \mathbf{1 3}\right]$ (adapted)). Suppose $C(\hat{\omega}, \rho)$ is a $q k$ private implementation of $f$ with encoder $I$ and decoder $O$, where $C$ makes an $\ell$-local use of its randomness, and uses at most $m$ bits of randomness. Let $G:\{0,1\}^{n} \rightarrow\{0,1\}^{m}$ be a strong $(r, k, q)$ robust linear PRG with $r \geq k \cdot \max (\ell, q)$. Then, the circuit $C^{\prime}$ defined by $C^{\prime}\left(\hat{\omega}, \rho^{\prime}\right)=C\left(\hat{\omega}, G\left(\rho^{\prime}\right)\right)$ is a k-private implementation of $f$ with encoder $I$ and decoder $O$ which uses $n$ random bits.

The proof of Theorem 1 is based on showing that the view of any adversary who attacks with $t$ probes an implementation in which the randomness is generated by a PRG, can be simulated given the view of an adversary with at most $q t$ probes who attacks an implementation with a true source of randomness; see Figure 3 for an illustration.

In Appendix B.3 we provide a proof that is essentially the same as in $\left[\mathrm{IKL}^{+} 13\right.$, Theorem 30$]$, except that we use our slightly weaker definition of robustness. We recall the main steps of the proof below. We start with the following Lemma, which is similar to [IKL ${ }^{+} 13$, Lemma 29]. As illustrated in Figure 2, any output of at most $r-q|S|$ bits of the robust PRG can be replaced by a TRNG and any set $S$ of at most $k$ probes in the PRG can be perfectly simulated using a subset $T$ of the output with $|T| \leq q|S|$. This means that probing $|S|$ probes within the PRG is not better for the adversary than probing $q|S|$ outputs of the TRNG. To simplify notation, we will use $G$ to denote both the function computed by a robust PRG and its circuit implementation. For a set $S$ of $k$ wires in $G$, we denote by $G_{S}$ the value of these wires; similarly, for a subset $T$ of output bits of $G$, we denote by $G_{T}$ the values of these output bits.

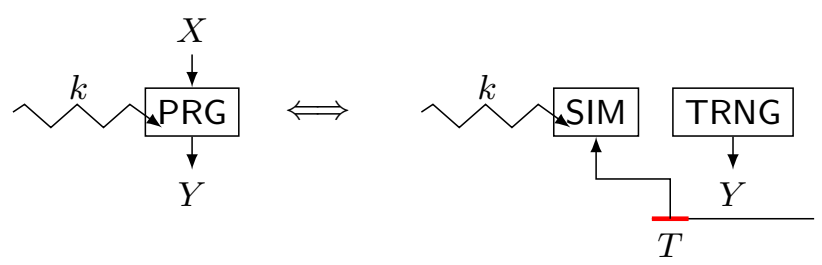

Fig. 2. With a strong $(r, k, q)$-robust PRG, any output of at most $r-q|S|$ bits of the PRG can be replaced by a TRNG and any set $S$ of at most $k$ probes can be perfectly simulated using a subset $T$ of the output with $|T| \leq q|S|$. 
Lemma 3 (Robust PRG). Let $G:\{0,1\}^{n} \rightarrow\{0,1\}^{m}$ be a strong $(r, k, q)$-robust linear PRG with $r \geq k q$. Let $S$ be any set of at most $k$ wires in $G$. Let $L \subset[m]$ be any subset of $r-q|S|$ bits. There exists a subset $T$ with $|T| \leq q|S|$ such that the distribution of $Y=G_{L \cup T}(X)$ is uniform in $\{0,1\}^{|L \cup T|}$ when $X \leftarrow\{0,1\}^{n}$ and moreover $G_{S}(X)$ can be efficiently simulated given $Y_{T}$ only.

Thanks to Lemma 3 we can now prove Theorem 1. As illustrated in Figure 3, we can simulate any $t$ probes within the PRG with a simulator SIM that uses $q t$ random bits from the TRNG (see Fig. 2); these $q t$ random bits can actually be queried by probing the original circuit $C$. This shows that when probing the PRG in $C^{\prime}$ the adversary does not learn more than by probing the circuit $C$ with true randomness, as required; see Appendix B.3 for the details.
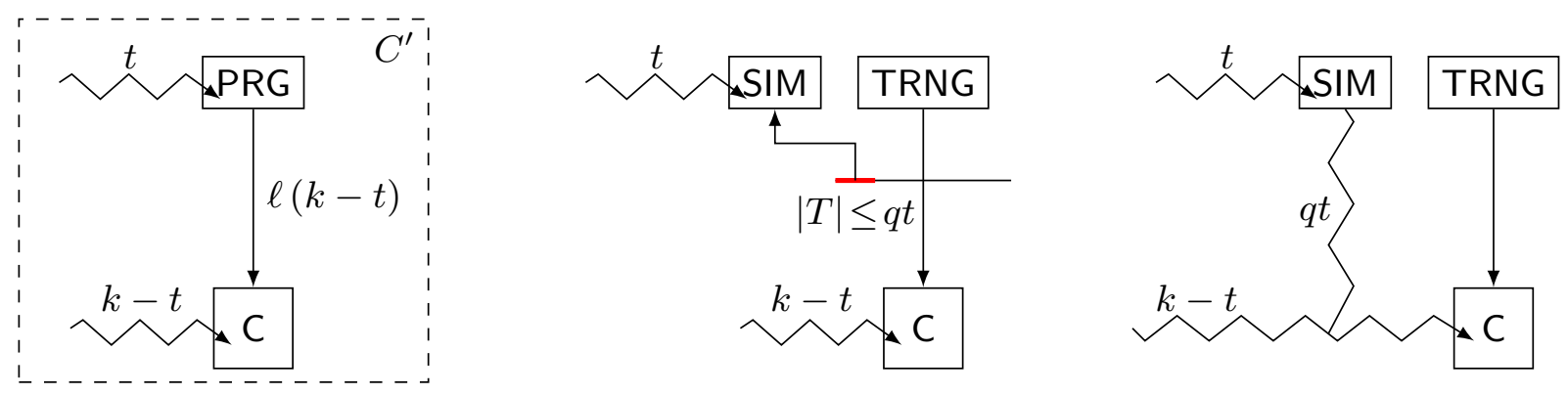

Fig. 3. Security proof when plugging a PRG into a private circuit.

\subsection{Locality refreshing}

As recalled in Theorem 1, the $r$-wise independence parameter $r$ of the PRG depends on the randomness locality $\ell$ of the circuit (see Definition 7). The goal is therefore to minimize the parameter $\ell$. In the original ISW construction, the parameter $\ell$ would grow linearly with the circuit size; namely some wires can depend on almost all the randoms used in the circuit. To keep a small $\ell=\mathcal{O}\left(t^{2}\right)$, the authors of [IKL $\left.{ }^{+} 13\right]$ use a mask refreshing at the end of each ISW gadget. Such locality refreshing, that we denote by LR, proceeds as described in Algorithm 1; see Figure 4 for an illustration.

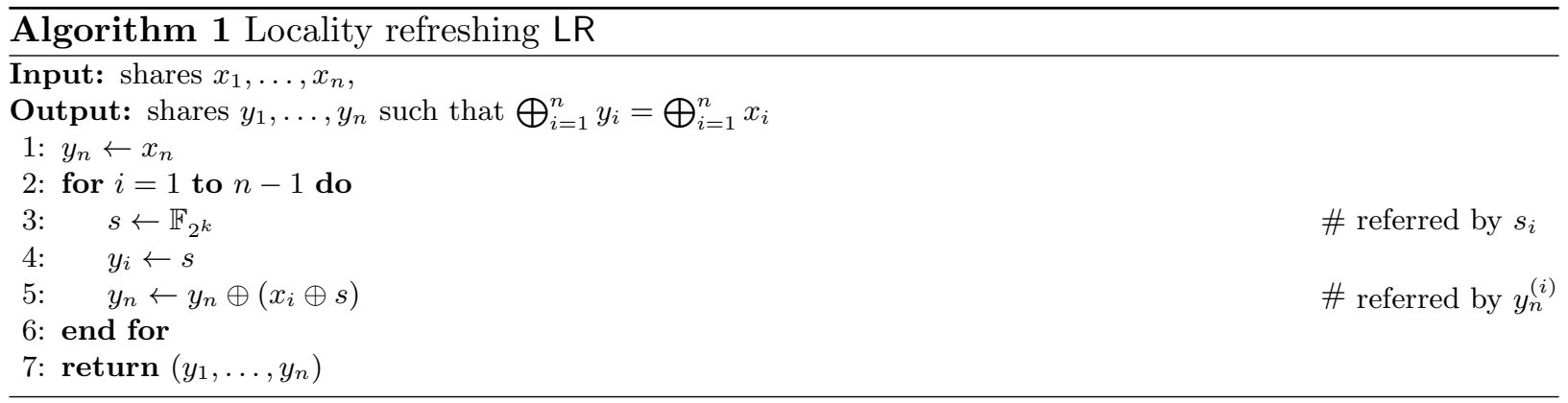

At the end of the algorithm, we have $y_{i}=s_{i}$ for all $1 \leq i \leq n-1$, and $y_{n}=x \oplus s_{1} \oplus \cdots \oplus s_{n-1}$ for the secret $x=x_{1} \oplus \cdots \oplus x_{n}$. Therefore one can show recursively over the circuit that the internal variables of the ISW multiplication depend on at most $\ell=\mathcal{O}\left(t^{2}\right)$ randoms, and this actually holds for any variable in the circuit. The following Lemma shows that the LR gadget is PINI, so that it can be included in a circuit without degrading its security.

Lemma 4 (PINI security of LR). Let $\left(x_{i}\right)_{1 \leq i \leq n}$ be the input shares of the mask refreshing Algorithm LR. For any $t \in \mathbb{N}$, any set of $t$ intermediate variables and any subset $\mathcal{O}$ of output 


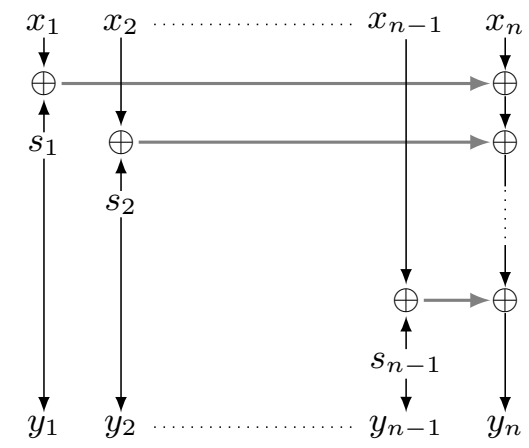

Fig. 4. Locality refreshing algorithm.

indices, there exists a subset $I \subset[1, n]$ of indices such the $t$ intermediate variables and the output shares $y_{\mid \mathcal{O}}$ can be perfectly simulated from the input shares $x_{\mid I \cup \mathcal{O}}$, with $|I| \leq t$.

Proof. We consider the following simple gadget $G:\left(x_{1}, x_{n}\right) \rightarrow\left(s_{1}, x_{n} \oplus\left(x_{1} \oplus s_{1}\right)\right)$, where $s_{1}$ is a random value. We start by showing that in Gadget $G$, we can always simulate $t$ probes and $|\mathcal{O}|$ output variables from the input shares $x_{\mid I \cup \mathcal{O}}$, with $|I| \leq t$.

If $t+|\mathcal{O}| \geq 2$, we can let $I=\{1, n\} \backslash \mathcal{O}$ which gives $I \cup \mathcal{O}=\{1, n\}$ and all variables can be simulated from the input shares $x_{\mid I \cup \mathcal{O}}$. Moreover we have $|I|=|\{1, n\} \backslash \mathcal{O}| \leq 2-|\mathcal{O}| \leq t$. If $t+|\mathcal{O}|=1$, we distinguish two cases. If $|\mathcal{O}|=1$ and $t=0$, then we can simulate either $s_{1}$ or $x_{n} \oplus\left(x_{1} \oplus s_{1}\right)$ by generating a random value. If $t=1$ and $|\mathcal{O}|=0$, we can simulate $x_{1}$ or $x_{n}$ with $I=\{1\}$ or $I=\{n\}$; the other variables can be simulated by a random value.

We now consider the following gadget $G_{i}$ for $1 \leq i \leq n-1$ :

$$
G_{i}:\left(x_{1}, \ldots, x_{i}, \ldots, x_{n}\right) \rightarrow\left(x_{1}, \ldots, x_{i-1}, s_{i}, x_{i+1}, \ldots, x_{n} \oplus\left(x_{i} \oplus s_{i}\right)\right)
$$

which is similar to Gadget $G$, but with $n$ input shares instead of 2 , and $n-2$ unmodified input shares. As previously, we can always simulate $t$ probes and $|\mathcal{O}|$ output variables from the input shares $x_{\mid I \cup \mathcal{O}}$, with $|I| \leq t$. This implies that the gadget $G_{i}$ is PINI. Since the LR gadget is the composition of $G_{1}, \ldots, G_{n-1}$, from Proposition 2 the LR gadget is also PINI.

In $\left[\mathrm{IKL}^{+} 13\right]$ the LR algorithm is then applied after each ISW gadget. In particular, for the SecMult gadget recalled in Appendix B.2, we obtain the following SecMultFLR gadget. Since the original SecMult is $t$-SNI, the SecMultFLR gadget is also $t$-SNI. The same LR algorithm is applied after the Xor gadget and the FullRefresh gadgets (see Appendix B.2).

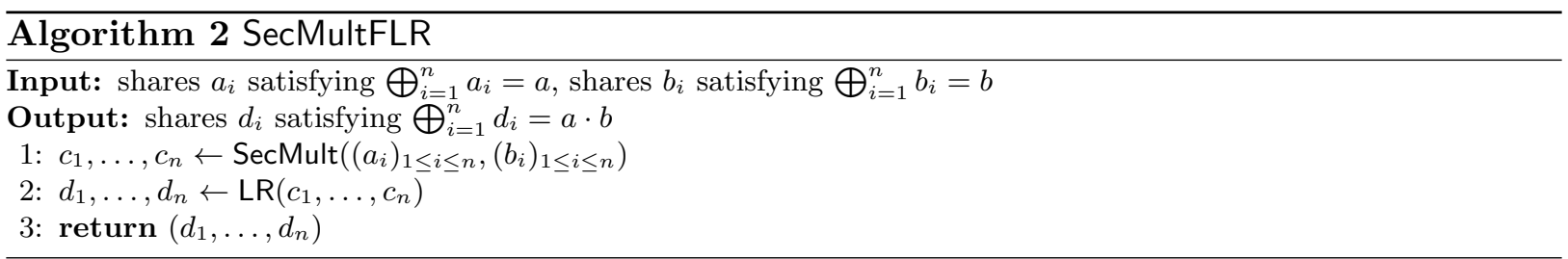

Application to private circuits. We recall Claim 31 and Corollary 32 from [IKL $\left.{ }^{+} 13\right]$; we also recall the proof in Appendix B.4. We use the notation $f(\lambda)=\tilde{\mathcal{O}}(g(\lambda))$ if $f(\lambda)=\mathcal{O}\left(g(\lambda) \log ^{k} \lambda\right)$ for some $k \in \mathbb{N}$. We assume that the circuit size $s(\lambda)$ and the number of probes $t(\lambda)$ are both polynomial in the security parameter $\lambda$.

Lemma 5 (Private circuit with PRG $\left[\mathbf{I K L}^{+} \mathbf{1 3}\right]$ ). Any function $f$ with circuit size $s$ admits a t-private implementation $(I, C, O)$ with the canonical encoder $I$ and decoder $O$, where $C$ uses 
$\mathcal{O}\left(t^{2} s\right)$ random bits and makes an $\ell=\mathcal{O}\left(t^{2}\right)$-local use of its randomness. Consequently, $f$ admits a t-private implementation $\left(I, C^{\prime}, O\right)$, where $C^{\prime}$ uses $\tilde{\mathcal{O}}\left(t^{4}\right)$ bits of randomness, and runs in time $\tilde{\mathcal{O}}\left(t^{6} s\right)$, using the trivial construction. Using the expander graph construction, for any $\varepsilon>0$, it uses $\mathcal{O}\left(t^{3+\varepsilon}\right)$ random bits and runs in time $\tilde{\mathcal{O}}\left(t^{2} s\right)$.

\subsection{Composing $\ell$-local gadgets}

In this section we provide an explicit definition of locality for a gadget, so that the locality property can be composed over a full circuit (as for the PINI definition for security against probing). As in $\left[\mathrm{IKL}^{+} 13\right]$, the basic technique is to perform a locality refresh (such as Algorithm 1 ) of the output of each gadget. We say that a set of wires $\left(y_{i}\right)_{1 \leq i \leq n}$ is locality refreshed if $y_{i}=s_{i}$ for all $1 \leq i \leq n-1$, for randoms $s_{i}$, and $y_{n}=y \oplus s_{1} \oplus \cdots \oplus s_{n-1}$, where $y$ is the original unmasked variable. In the definition below of gadget locality, we take into account the randomness of the (locality refreshed) inputs.

Definition 8 ( $\ell$-local gadget). Let $G$ be a gadget whose output is locality refreshed. Consider the circuit $C$ where $G$ is given locality refreshed inputs $x_{\star, \star}$. Let $\rho$ be the randomness used by $C$, including the randomness from the inputs. The gadget $G$ is said to make an $\ell$-local use of its randomness if $C$ makes an $\ell$-local use of its randomness $\rho$

Theorem 2 (Composition of $\ell$-local gadgets). Any composite gadget made of $\ell$-local gadgets is $\ell$-local.

Proof. We consider $m$ gadgets $G_{1}, \cdots, G_{m}$ that we order as a direct acyclic graph from output to input in a reverse topological sort order. We assume that each gadget $G_{i}$ makes an $\ell$-local use of its randomness, with locality refreshed outputs. We prove by recurrence on $n$ that the composition of $\ell$-local gadgets is $\ell$-local.

If $n=1$, then there is only one gadget and this is straightforward since by assumption the gadget is $\ell$-local. Now we assume that the composition of gadgets $G_{1}, \cdots, G_{n}$ is $\ell$-local and we prove that the composition of gadgets $G_{1}, \cdots, G_{n+1}$ is still $\ell$-local. Since the composition of gadgets $G_{1}, \cdots, G_{n}$ is $\ell$-local, and since by definition the inputs of the gadget $G_{n}$ are locality refreshed because they correspond to outputs of Gadget $G_{n+1}$ which are locality refreshed, we get that the composition of both parts $G_{n+1}$ and $G_{1}, \cdots, G_{n}$ does not increase the global locality. Namely, the global locality corresponds to the maximum locality between both parts. Since the composition of gadgets $G_{1}, \cdots, G_{n}$ is $\ell$-local and since Gadget $G_{n+1}$ is also $\ell$-local, the maximum locality is $\ell$ and the composition of gadgets $G_{1}, \cdots, G_{n+1}$ is $\ell$-local.

In the above definition, in order to determine the locality $\ell$ of a gadget, we must therefore assume that it receives locality refreshed inputs, and the randomness from this locality refreshed inputs must be taken into account when computing $\ell$. Below we provide an example with the Xor gadget; the Xor gadget takes as input $a_{i}$ and $b_{i}$ for $1 \leq i \leq n$, and returns $c_{i}=a_{i} \oplus b_{i}$ for all $1 \leq i \leq n$.

Lemma 6 (Locality of Xor). The Xor gadget followed by a locality refresh makes an $\ell$-local use of its randomness, with $\ell=2(n-1)$.

Proof. The gadget takes as input $a_{i}$ and $b_{i}$ for $1 \leq i \leq n$, and then computes $c_{i}=a_{i} \oplus b_{i}$ for all $1 \leq i \leq n$, and finally $d_{n, j}=c_{n} \oplus\left(\oplus_{i=1}^{j} a_{i} \oplus b_{i} \oplus s_{i}\right)$ for $1 \leq j \leq n-1$, with outputs $d_{i}=s_{i}$ for $1 \leq i \leq n-1$ and $d_{n}=d_{n, n-1}$. We must consider $a_{i}=s_{i}^{(a)}$ for $1 \leq i \leq n-1$ and $a_{n}=a \oplus s_{1}^{(a)} \oplus \cdots \oplus s_{n-1}^{(a)}$, and similarly for $b_{i}$. Therefore $c_{n}$ depends on $2(n-1)$ randoms, while $d_{n, j}$ depends on $2(n-1)-j$ randoms, which proves the lemma.

We also compute the concrete locality $\ell$ of the SecMultFLR algorithm introduced above; in $\left[\mathrm{IKL}^{+} 13\right]$ only the asymptotic bound $\ell=\mathcal{O}\left(n^{2}\right)$ was proved. Such concrete locality computations will be important when implementing the countermeasure for AES in Section 5; namely for a 
locality $\ell$, from Theorem 1 the $r$-wise independence parameter of the PRG must be set to $r=\ell t$ for security against $t$ probes. We refer to Appendix B.4 for the proof.

Lemma 7 (Locality of SecMultFLR). The SecMult algorithm followed by a final locality refresh (SecMultFLR) is an $\ell$-local gadget with $\ell=n^{2} / 4+5 n / 2-c$, where $c=3$ for even $n$, and $c=11 / 4$ for odd $n$.

\section{Improving the locality of the multiplication gadget}

In this section we describe two variants of the SecMult algorithm that improve the randomness locality of $t$-private circuits from $\ell=\mathcal{O}\left(t^{2}\right)$ to $\ell=\mathcal{O}(t)$. We show that this decreases the randomness complexity of private circuits from $\tilde{\mathcal{O}}\left(t^{4}\right)$ to $\tilde{\mathcal{O}}\left(t^{3}\right)$ using the trivial robust PRG construction. For our two new algorithms SecMultILR and SecMultILR2, we summarize in Table 3 below the number of required randoms and their locality $\ell$. Since these randoms are eventually generated by a PRG, one should minimize their locality $\ell$. We introduce SecMultILR first because the $t$-SNI proof of SecMultILR2 is significantly more complex.

\begin{tabular}{|l|c|c|c|c|}
\cline { 2 - 5 } \multicolumn{1}{c|}{} & SecMult [ISW03] & SecMultFLR [IKL $\left.{ }^{+} 13\right]$ & SecMultILR & SecMultILR2 \\
\hline Number of randoms & $n(n-1) / 2$ & $n(n-1) / 2+n-1$ & $n(n-1)$ & $n(n-1) / 2+n-1$ \\
\hline Locality $\ell$ & - & $n^{2} / 4+5 n / 2-c$ & $4 n-5$ & $4 n-6$ \\
\hline Security & $t$-SNI & $t$-SNI & $t$-SNI & $t$-SNI \\
\hline
\end{tabular}

Table 3. Summary of the multiplication gadgets, their locality and security. We have $c=3$ for even $n$, and $c=11 / 4$ for odd $n$.

\subsection{First Construction with Internal Locality Refreshing (SecMultILR)}

We describe below a variant of the SecMultFLR algorithm with locality $\ell=\mathcal{O}(t)$ instead of $\ell=$ $\mathcal{O}\left(t^{2}\right)$. Our new SecMultILR is described below. The idea is to process the ISW matrix differently. In the original SecMult the final encoding is obtained by summing over all rows of the $n \times n$ ISW matrix. Instead we compute the partial sums over the rows of the successive $j \times j$ submatrices for $2 \leq j \leq n$. At each step we perform a locality refreshing of the $j$ shares of the partial sum. In particular, the output of the algorithm is locality refreshed, so there is no need to apply the LR algorithm again.

We see that lines 6 to 9 are the same as in the original SecMult (see Appendix B.2), except that they are processed in a different order, since the loop starts with $j$ instead of $i$. This implies that at Step 10 we have processed the $j \times j$ submatrix of the ISW matrix, and therefore the first $j$ shares $c_{i}$ must satisfy the equality:

$$
c_{1} \oplus \cdots \oplus c_{j}=\left(a_{1} \oplus \cdots \oplus a_{j}\right) \cdot\left(b_{1} \oplus \cdots \oplus b_{j}\right)
$$

From lines 11 to 15 we then perform a locality refresh of these $j$ shares $\left(c_{i}\right)_{i=1}^{j}$ using new randoms $s_{i j}$; therefore after the locality refresh the new shares $c_{i}$ satisfy the same equality (2), but now they only depend on the $j-1$ randoms $s_{i j}$ for $1 \leq i \leq j-1$, and not on the $r_{i j}$ 's. This implies that at the next step of the loop (for index $j+1$ ), the shares $c_{i}$ will only depend on a linear number of randoms $r_{i j}$, instead of quadratic in the original SecMult. Thanks to these internal locality refreshings, the new locality parameter becomes $\ell=\mathcal{O}(t)$ instead of $\ell=\mathcal{O}\left(t^{2}\right)$; we provide the proof of the locality lemma below in Appendix C.1. We also prove the completeness of our multiplication algorithm in Appendix C.2, and we show that it remains $t$-SNI in Appendix C.3.

Lemma 8 (Locality of SecMultILR). The SecMultILR algorithm is an $\ell$-local gadget with $\ell=$ $4 n-5$ for $n \geq 3$. 


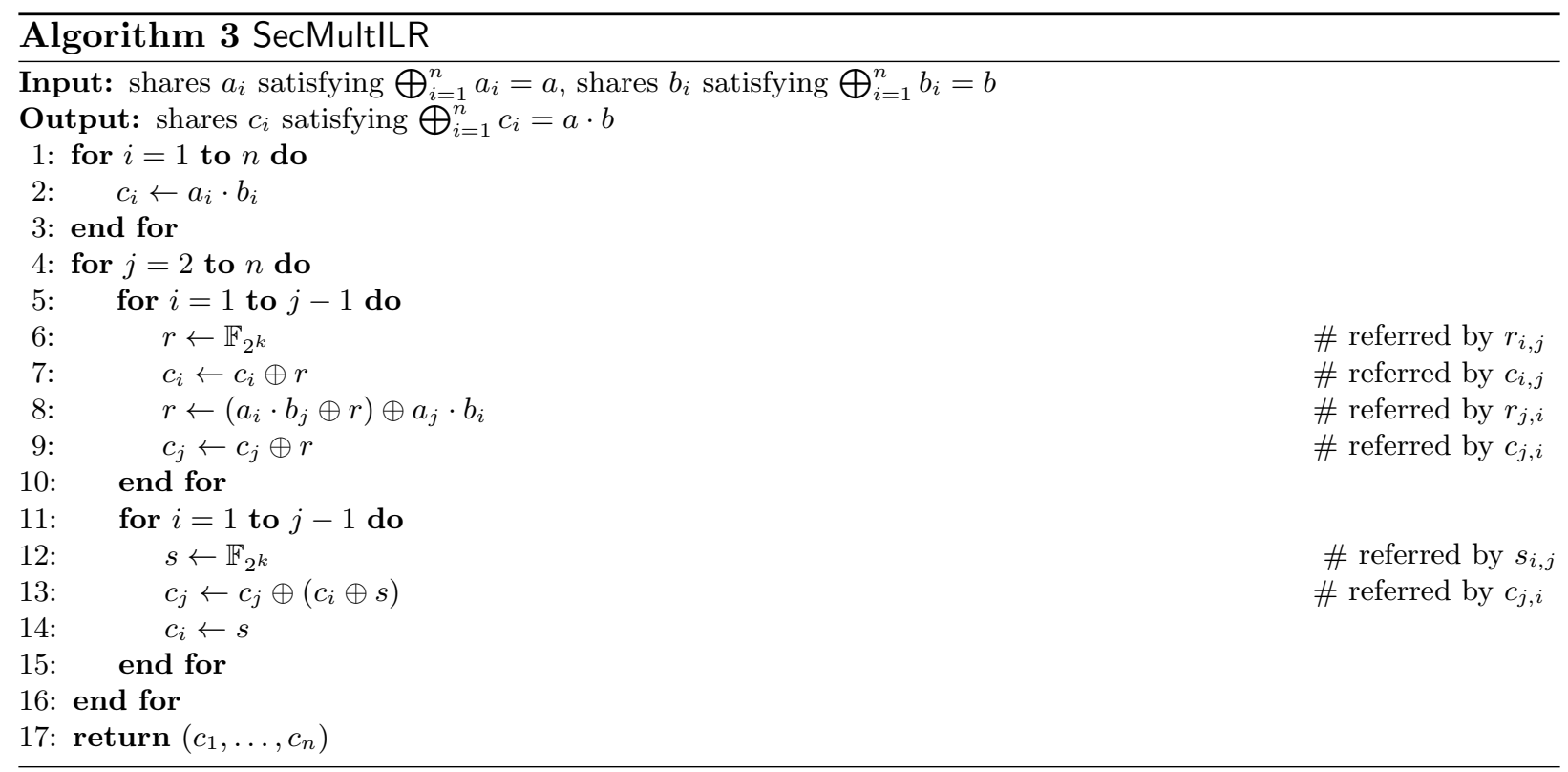

Theorem 3 (Completeness of SecMultILR). The SecMultILR algorithm, when taking $a_{1}, \ldots, a_{n}$ and $b_{1}, \ldots, b_{n}$ as inputs, outputs $c_{1}, \ldots, c_{n}$ such that $c_{1} \oplus \cdots \oplus c_{n}=\left(a_{1} \oplus \ldots \oplus a_{n}\right) \cdot\left(b_{1} \oplus \ldots \oplus b_{n}\right)$.

Theorem 4 ( $t$-SNI of SecMultILR). The SecMultILR algorithm is $t$-SNI for any $1 \leq t \leq n-1$.

One can therefore use a robust PRG with $r$-wise independence parameter $r=\ell \cdot t=\mathcal{O}\left(t^{2}\right)$ instead of $r=\mathcal{O}\left(t^{3}\right)$ in [IKL $\left.{ }^{+} 13\right]$. With the trivial construction of xoring $t+1$ PRGs, the number of input randoms in the finite field becomes $r \cdot(t+1)=\mathcal{O}\left(t^{3}\right)$ instead of $\mathcal{O}\left(t^{4}\right)$. This gives the following lemma, which improves over Lemma 5 from $\left[\mathrm{IKL}^{+} 13\right]$.

Lemma 9 (Efficiency properties of SecMultILR). Any function of circuit size $s$ admits a $t$ private implementation $(I, C, O)$ with the canonic encoder $I$ and decoder $O$, where $C$ uses $\tilde{\mathcal{O}}\left(t^{3}\right)$ bits of randomness using the trivial construction, and runs in time $\tilde{\mathcal{O}}\left(s \cdot t^{5}\right)$.

\subsection{Second construction with less randomness (SecMultILR2)}

We describe in Appendix C.4 a variant called SecMultILR2 of the previous algorithm, that achieves the same locality $\ell$ as SecMultILR but with roughly half as many randoms. It uses the same number of randoms as SecMultFLR from [IKL $\left.{ }^{+} 13\right]$, but with locality $\mathcal{O}(t)$ instead of $\mathcal{O}\left(t^{2}\right)$. Therefore it is strictly better than both SecMultFLR and SecMultILR; see Table 3. We provide the proof of the locality lemma in Appendix C.5, the proof of completeness in Appendix C.6 and the proof of $t$-SNI security in Appendix C.7.

Lemma 10 (Locality of SecMultILR2). The SecMultILR2 gadget uses $\ell$-local randomness, with $\ell=4 n-6$ for $n \geq 3$.

Theorem 5 (Completeness of SecMultILR2). The SecMultILR2 algorithm, when taking $a_{1}, \ldots, a_{n}$ and $b_{1}, \ldots, b_{n}$ as inputs, outputs $c_{1}, \ldots, c_{n}$ such that $c_{1} \oplus \cdots \oplus c_{n}=\left(a_{1} \oplus \ldots \oplus a_{n}\right) \cdot\left(b_{1} \oplus \ldots \oplus b_{n}\right)$.

Theorem 6 ( $t$-SNI of SecMultILR2). The SecMultILR2 is $t$-SNI for any $1 \leq t \leq n-1$.

\subsection{Formal verification of locality and security}

We have performed a formal verification of the above locality and security lemmas, using the CheckMasks tool [Cor18]. In this framework the circuit is represented as nested lists, which leads to a simple and concise implementation in Common Lisp. The $t$-SNI property of SecMult was 
already formally verified up to $n=5$ shares in [Cor18]; namely the verification has exponential complexity in $n$. In tables 4 and 5 we have performed a similar verification of theorems 4 and 6 for SecMultILR and SecMultILR2 respectively; although we could only verify the security up to $n=5$, this brings some confidence in the correctness of our $t$-SNI security proofs.

\begin{tabular}{|c|c|c|c|c|}
\hline$n$ & \#variables & \#tuples & Security & Time \\
\hline 3 & 39 & 741 & $\checkmark$ & $\varepsilon$ \\
\hline 4 & 72 & 59,640 & $\checkmark$ & $3 \mathrm{~s}$ \\
\hline 5 & 115 & $6,913,340$ & $\checkmark$ & $12 \mathrm{~min}$ \\
\hline
\end{tabular}

Table 4. SecMultILR: formal verification of Theorem 4 , for small values of $n$.

\begin{tabular}{|c|c|c|c|c|}
\hline$n$ & \#variables & \#tuples & Security & Time \\
\hline 3 & 36 & 630 & $\checkmark$ & $\varepsilon$ \\
\hline 4 & 63 & 39,711 & $\checkmark$ & $2 \mathrm{~s}$ \\
\hline 5 & 97 & $3,464,840$ & $\checkmark$ & $5 \mathrm{~min}$ \\
\hline
\end{tabular}

Table 5. SecMultILR2: formal verification of Theorem 6 , for small values of $n$.

Similarly the locality is easy to compute in the framework of CheckMasks; it requires only a few lines of Common Lisp code. We provide in Table 6 the formal computation of the locality $\ell$ for the SecMultFLR, SecMultILR and SecMultILR2 algorithms; it matches the formulas from Table 3 . This time the computation is polynomial-time in $n$, so we can compute the locality for large values of $n$. We provide the source code for both locality and security verification in [Cor19a].

\begin{tabular}{|l|c|c|c|c|c|c|c|c|c|c|c|c|c|}
\hline Number of shares $n$ & 3 & 4 & 5 & 6 & 7 & 8 & 9 & 10 & 11 & 12 & 13 & 14 & 15 \\
\hline SecMultFLR & 7 & 11 & 16 & 21 & 27 & 33 & 40 & 47 & 55 & 63 & 72 & 81 & 91 \\
\hline SecMultILR & 7 & 11 & 15 & 19 & 23 & 27 & 31 & 35 & 39 & 43 & 47 & 51 & 55 \\
\hline SecMultILR2 & 6 & 10 & 14 & 18 & 22 & 26 & 30 & 34 & 38 & 42 & 46 & 50 & 54 \\
\hline
\end{tabular}

Table 6. Formal computation of the locality parameter $\ell$, as a function of the number of shares $n$.

\section{Private Circuits with Multiple PRGs without Robustness}

In the previous section we have described two variants of SecMult where following the [IKL $\left.{ }^{+} 13\right]$ paradigm a single robust PRG is used to generate all the randoms from the circuit; by improving the locality parameter from $\ell=\mathcal{O}\left(t^{2}\right)$ to $\ell=\mathcal{O}(t)$, we have decreased the number of input random bits from $\tilde{\mathcal{O}}\left(t^{4}\right)$ to $\tilde{\mathcal{O}}\left(t^{3}\right)$, that is independent of the circuit size $s$ (up to logarithmic factors). In this section, we show that by using multiple independent PRGs instead of a single one, the robustness property of the PRG is not required anymore, and therefore much more efficient PRG constructions can be used; this allows to decrease the randomness complexity of private circuits down to $\tilde{\mathcal{O}}\left(t^{2}\right)$.

We start with a simple observation. In the security proof of ISW, if the attacker probes a given random $r_{i j}$ in some SecMult gadget, then it is easy to see that we could give away to the attacker not only the probed $r_{i j}$, but actually all randoms $r_{i j}^{(k)}$ for the same $i, j$ in all other SecMult gadgets $k$; namely in the ISW security proof with global index $I$, one would have $i \in I$, and therefore each $r_{i j}^{(k)}$ would then be simulated by letting $r_{i j}^{(k)} \leftarrow \mathbb{F}$ as in the original circuit, so it could be given to the attacker without requiring the knowledge of more input shares.

Now assume that for every pair $(i, j)$ we use an independent PRG to generate the randoms $r_{i j}^{(k)}$ for all gadgets $k$. In that case the attacker has no advantage in probing the intermediate variables of the PRG circuit, since in our extended probing model he could get all corresponding randoms $r_{i j}^{(k)}$ with a single probe anyway. Therefore when each $r_{i j}$ has a dedicated PRG (see Figure 5 for an illustration), the robustness property of the PRG is not required anymore, and we can use a simple PRG with $r$-wise independence only, as for example the PRG based on polynomial evaluation from Section 2.3. 


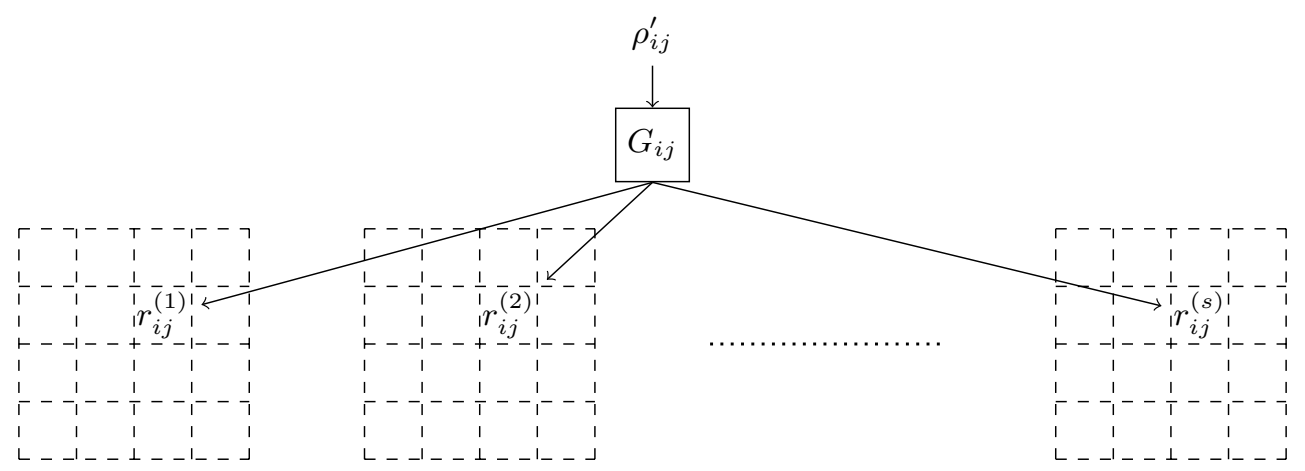

Fig. 5. In Construction 1 , each $r_{i j}$ has its dedicated PRG across all gadgets, from a random seed $\rho_{i j}^{\prime}$.

Moreover, if a mask locality refreshing is performed at the end of each multiplication gadget, it is easy to see that any intermediate variable of the circuit can depend on at most a single random $r_{i j}^{(k)}$ for a fixed $i, j$, and therefore the locality with respect to each randomness subset $\rho_{i j}=\left\{r_{i j}^{(k)}: 1 \leq k \leq s\right\}$ is $\ell=1$; this is because the locality refresh at the end of each multiplication gadget cancels the dependence on the internal $r_{i j}^{(k)}$. In that case, with $t$ probes on intermediate variables the adversary can get information on at most $t$ randoms within such set. Therefore these randoms can be generated by a PRG with $r$-wise independence parameter $r=t$. Since the robustness property is not required, we can use a PRG based on polynomial evaluation that requires only $r=t$ coefficients in a finite field, and therefore $\tilde{\mathcal{O}}(t)$ random bits per PRG. Since there are $\mathcal{O}\left(t^{2}\right)$ randoms $r_{i j}$, we need $\mathcal{O}\left(t^{2}\right)$ independent PRGs to generate all of them, and the total number of input random bits is therefore $\tilde{\mathcal{O}}\left(t^{3}\right)$, as in our single PRG constructions from Section 3. Note that the time to generate a pseudo-random is now $\tilde{\mathcal{O}}(t)$, instead of $\tilde{\mathcal{O}}\left(t^{3}\right)$ in Section 3.

We can improve the above randomness complexity as follows. Firstly, we observe as previously that in the security proof of ISW, whenever the attacker probes a random $r_{i j}$, we can actually give to the attacker the complete row of $r_{i j}$ 's, that is for a given $i$, all $r_{i j}$ with $i<j \leq n$; and more generally, for a fixed $i$, all randoms $r_{i j}^{(k)}$ with $i<j \leq n$ in all SecMult gadgets $k$. Therefore as previously we can use for each $1 \leq i<n$ a dedicated PRG to generate all $r_{i j}^{(k)}$ for all $i<j \leq n$ in all gadgets $k$, without needing the robustness property. Since we generate the complete row of $r_{i j}$ 's (see Fig. 8 for an illustration), we only need $\mathcal{O}(t)$ independent PRGs, instead of $\mathcal{O}\left(t^{2}\right)$.

Moreover, if we perform internal mask refreshing as in the SecMultILR algorithm from Section 3 (instead of only at the end of the SecMult gadget), then no intermediate variable can depend on two distinct $r_{i j}$ 's in the same row $i$. This implies that the locality with respect to the randomness subset $\rho_{i}=\left\{r_{i j}^{(k)}: i<j \leq n, 1 \leq k \leq s\right\}$ is still equal to 1 . Therefore a PRG can be used to generate all $r_{i j}^{(k)}$ from a given row $i$ in all gadgets $k$, still with $r$-wise independence parameter $r=t$. Since we need only $\mathcal{O}(t)$ independent PRGs instead of $\mathcal{O}\left(t^{2}\right)$ previously, the number of input random bits goes down to $\tilde{\mathcal{O}}\left(t^{2}\right)$, while the time to generate a pseudo-random is still $\tilde{\mathcal{O}}(t)$. Asymptotically this is the most efficient technique (see Table 1), and also the most efficient in practice (see Section 5 for our implementation results on AES).

\subsection{Security with multiple PRGs}

The following lemma shows that the PRG robustness is not needed when the PRG generates only a subset $\rho$ of the randomness, and the adversary can get $\rho$ with a single probe; the lemma is analogous to Theorem 1 for a single robust PRG. We first consider a circuit $C$ where we split the randomness in two parts $\rho$ and $\bar{\rho}$, where only the randomness $\rho$ will be replaced by pseudo-randoms. We consider an extended security model in which the attacker can get $\rho$ with a single probe. Intuitively probing the PRG that generates $\rho$ does not help the attacker, since in the extended security model he can get $\rho$ with a single probe. 
Lemma 11 (Security from $r$-wise independent PRG). Suppose $C$ is a t-private implementation of $f$ with encoder $I$ and decoder $O$, where $C(\hat{\omega}, \rho, \bar{\rho})$ uses $m$ random bits $\rho$ and makes an $\ell$-local use of its randomness $\rho$, and the adversary can obtain $\rho$ with a single probe. Let $G:\{0,1\}^{n_{r}} \rightarrow\{0,1\}^{m}$ be a linear $\ell t$-wise independent PRG. Then, the circuit $C^{\prime}$ defined by $C^{\prime}\left(\hat{\omega}, \rho^{\prime}, \bar{\rho}\right)=C\left(\hat{\omega}, G\left(\rho^{\prime}\right), \bar{\rho}\right)$ is a t-private implementation of $f$ with encoder $I$ and decoder $O$ which uses $n_{r}$ random bits $\rho^{\prime}$ and random $\bar{\rho}$.

Proof. We show that the view of an adversary $A^{\prime}$ who attacks $C^{\prime}\left(\hat{\omega}, \rho^{\prime}, \bar{\rho}\right)$ by probing a set $S$ of $t^{\prime} \leq t$ wires in $G$ and a set of $P$ of $t-t^{\prime}$ wires in $C$ is independent of the secret input $\omega$. Since $C$ is $t$-private, it suffices to show that that the view of $A^{\prime}$ can be simulated given the view of an adversary $A$ who probes at most $t$ wires in $C(\hat{\omega}, \rho, \bar{\rho})$, and who can obtain the randomness $\rho$ with a single probe.

Since $C$ makes an $\ell$-local use of its randomness $\rho$, the $t-t^{\prime}$ probes from the set $P$ in the circuit $C$ can depend on at most $\ell\left(t-t^{\prime}\right) \leq \ell t$ bits of $\rho$. More precisely, for any $\hat{\omega}$ and $\bar{\rho}$, let $Q_{\hat{\omega}, \bar{\rho}}(\rho)=C_{P}(\hat{\omega}, \rho, \bar{\rho})$ be the value of these probes; the function $Q_{\hat{\omega}, \bar{\rho}}$ depends on at most $\ell$ bits of $\rho$. Let $T \subset[1, m]$ be the corresponding subset of bits of $\rho$ on which $Q_{\hat{\omega}, \bar{\rho}}$ depends, with $|T| \leq \ell t$; we can write $Q_{\hat{\omega}, \bar{\rho}}(\rho)=Q^{\prime}\left(\rho_{T}\right)$, where $\rho_{T}$ is the corresponding subset of $\rho$.

We now proceed as follows. Instead of generating the PRG seed $X \leftarrow\{0,1\}^{n_{r}}$ and then the PRG output $G_{T}(X)$ corresponding to $T$, we can first generate the PRG output $\rho_{T} \leftarrow\{0,1\}|T|$ and then sample the PRG seed; this is possible because $G$ is a linear $\ell t$-wise independent PRG, and moreover $|T| \leq \ell t$. More precisely, as in the proof of Lemma 19, since $G$ is a linear $\ell t$-wise PRG, there exists a randomized simulator Sim that can perfectly sample the PRG input and therefore the probes within the PRG, given at most $\ell$ bits of PRG output; formally this means $\left(G_{S}(X), G_{T}(X)\right) \equiv\left(\operatorname{Sim}\left(\rho_{T}\right), \rho_{T}\right)$ where $X \leftarrow\{0,1\}^{n_{r}}$ and $\rho \leftarrow\{0,1\}^{m}$. We obtain:

$$
\left(G_{S}(X), Q^{\prime}\left(G_{T}(X)\right)\right) \equiv\left(\operatorname{Sim}\left(\rho_{T}\right), Q^{\prime}\left(\rho_{T}\right)\right)
$$

We now distinguish two cases. If the number of probes within the PRG is such that $t^{\prime} \geq 1$, then as in the proof of Lemma 19, we let $\operatorname{Sim}^{\prime}\left(\rho_{T}, v\right)=\left(\operatorname{Sim}\left(\rho_{T}\right), v\right)$ and we obtain:

$$
\left(G_{S}(X), Q^{\prime}\left(G_{T}(X)\right)\right) \equiv\left(\operatorname{Sim}\left(\rho_{T}\right), Q^{\prime}\left(\rho_{T}\right)\right) \equiv \operatorname{Sim}^{\prime}\left(\rho_{T}, Q^{\prime}\left(\rho_{T}\right)\right)
$$

which gives

$$
\left(G_{S}(X), Q_{I(\omega), \bar{\rho}}(G(X))\right) \equiv \operatorname{Sim}^{\prime}\left(\rho_{T}, Q_{I(\omega), \bar{\rho}}(\rho)\right) .
$$

In this case, the distribution to which Sim' is applied captures the view of an adversary $A$ who corrupts a set $T \cup P$ of wires in $C$, where $|P| \leq t-t^{\prime}$ and by definition $\rho_{T}$ can be obtained with a single probe, which gives a total of at most $t-t^{\prime}+1 \leq t$ probes in $C$. Since by assumption $C$ is $t$-private, this view is independent of the secret $\omega$. Since the distribution on the left hand side captures the view of $A^{\prime}$, it follows that the view of $A^{\prime}$ is also independent of $\omega$, as required.

In the second case, $G$ is not probed by the adversary $A^{\prime}$. Since $G$ is $\ell t$-wise independent and the view of $A^{\prime}$ depends on at most $\ell t$ bits of $\rho$, the view of $A^{\prime}$ is the same as the view of an adversary $A$ probing the same wires in $C$. More precisely, we have from $G_{T}(X) \equiv \rho_{T}$ :

$$
Q_{I(\omega), \bar{\rho}}(G(X)) \equiv Q_{I(\omega), \bar{\rho}}(\rho)
$$

As previously, the right hand side corresponds to the view of an adversary $A$ who corrupts a set $P$ of at most $t$ wires in $C$ and the distribution of the left hand side captures the view of $A^{\prime}$; therefore the view of $A^{\prime}$ is independent of $\omega$ also in the second case.

We now consider the main theorem where the circuit randomness $\rho$ can be split into $\left(\rho_{i}\right)_{i=1}^{k}$, and when considering each $\rho_{i}$ separately, the circuit $C$ makes an $\ell$-local use of $\rho_{i}$; moreover we assume that $C$ remains $t$-private even if the adversary can obtain each $\rho_{i}$ with a single probe. The proof follows from a recursive application of Lemma 11. 
Theorem 7 (Security with multiple PRGs). Suppose $C$ is a t-private implementation of $f$ with encoder $I$ and decoder $O$, where the circuit $C\left(\hat{\omega}, \rho_{1}, \ldots, \rho_{k}\right)$ uses for each $1 \leq i \leq k, m$ random bits $\rho_{i}$, and makes an $\ell$-local use of $\rho_{i}$, and the adversary can obtain each $\rho_{i}$ with a single probe. Let $G:\{0,1\}^{n_{r}} \rightarrow\{0,1\}^{m}$ be a linear $\ell$-wise independent $P R G$. Then, the circuit $C^{\prime}$ defined by $C^{\prime}\left(\hat{\omega}, \rho_{1}^{\prime}, \ldots, \rho_{k}^{\prime}\right)=C\left(\hat{\omega}, G\left(\rho_{1}^{\prime}\right), \ldots, G\left(\rho_{k}^{\prime}\right)\right)$ is a t-private implementation of $f$ with encoder $I$ and decoder $O$ which uses $k \cdot n_{r}$ random bits.

\subsection{Extended Security Model: PINI-R}

In Theorem 7 above we have considered an extended model of security, where the adversary can get any randomness subset $\rho_{i}$ in the circuit with a single probe. Therefore, we define a variant of the PINI notion from [CS18], called PINI-R, in which the adversary can also get access to a subset of the randoms in a gadget, using a single probe.

Definition 9 (PINI-R). Let $G$ be a gadget with input shares $x_{i, \star}$ and output shares $y_{i, \star}$. Let $\left(\rho_{i}\right)_{1 \leq i \leq n}$ be a partition of the randoms used by $G$. The gadget $G$ is PINI-R if for any $t_{1} \in \mathbb{N}$, any set of $t_{1}$ intermediate variables, any subset $\mathcal{O}$ of output indices and any subset $R \subset[1, n]$, there exists a subset $I \subset[1, n]$ of input indices with $|I| \leq t_{1}$ such that the $t_{1}$ intermediate variables, the output shares $y_{\mid \mathcal{O} \cup R, \star}$ and the randoms $\rho_{i}$ for $i \in R$ can be perfectly simulated from the input shares $x_{\mid I \cup \mathcal{O} \cup R, \star}$.

The following proposition is analogous to Proposition 1. It shows that if a gadget with $n=t+1$ shares is PINI-R, then a $t$-probing adversary learns nothing about the underlying secrets, even in an extended model of security where the adversary can get each randomness subset $\rho_{i}$ with a single probe. We provide the proof in Appendix D.1.

Proposition 4 (PINI-R security). Let $G$ be a gadget with input shares $x_{i, \star}$ and output shares $y_{i, \star}$ for $1 \leq i \leq n$. Let $\left(\rho_{i}\right)_{1 \leq i \leq n}$ be a partition of the randomness used by $G$. If $G$ is PINI- $R$, then $G$ is $(n-1)$-probing secure in an extended model of security where the adversary can get each $\rho_{i}$ with a single probe.

In the composition theorem below, the attacker can get the union of all corresponding subsets of randoms from all gadgets, still with a single probe; see Appendix D.2 for the proof.

Theorem 8 (Composition of PINI-R). Any composite gadget made of PINI-R composing gadgets $G_{i}$ for $i \in K$ is PINI-R, where for the composite gadget we take the randomness partition $\rho_{i}=\bigcup_{k \in K} \rho_{i}^{(k)}$ for $1 \leq i \leq n$.

It is straightforward to prove the PINI-R property of the locality refreshing algorithm from Section 2.6, with the randomness partition $\rho_{i}=\left\{s_{i}\right\}$ for $1 \leq i \leq n-1$. In Appendix D.3 we consider an analogous extension of the $t$-SNI property, called $t$-SNI-R, which we prove for the SecMult and SecMultILR constructions, and the corresponding FullRefresh. More precisely, we show that those gadgets remain secure in an extended model of security where the adversary can get all randoms $r_{i j}$ (and all randoms $s_{i j}$ for SecMultILR) for a given $i$ with a single probe. Moreover the "double-SNI" approach still works for the $t$-SNI-R and PINI-R notions. This implies that we can base our construction on $t$-SNI-R and PINI-R gadgets, and the resulting construction will be PINI-R. Note that the $t$-SNI security proof of SecMultILR2 is already complex, so we will not try to prove the $t$-SNI-R property of SecMultILR2; therefore we will use the multiple PRGs approach for SecMultFLR and SecMultILR only.

\subsection{Constant locality with respect to a randomness subset}

In this section we show that we can achieve constant locality, even $\ell=1$, when we consider different subsets of randomness. Therefore we first provide a definition of gadget locality with respect to a subset of the gadget randomness only (and excluding the randomness of the inputs, as opposed to Section 2.7), and then a locality composition theorem as in Section 2.7. 
Definition 10 ( $\ell$-local gadget with randomness subset). Let $G$ be a gadget and let $\rho$ be a subset of the randomness used by $G$. The gadget $G$ is said to make an $\ell$-local use of its randomness $\rho$ if any intermediate variable of $G$ depends on at most $\ell$ bits of $\rho$.

For example, the SecMult gadget makes a 1-local use of its randomness $\rho=\left\{r_{i j}\right\}$ for any $1 \leq i<j \leq n$; this is obvious, since $\rho$ contains a single random bit. We can now state our composition theorem for locality with respect to a randomness subset. It shows that the gadget locality $\ell$ is kept the same in the composite gadget, while the locality of the randoms used for output refreshing is equal to 3 with respect to each subset $\left\{s_{i}^{(k)}, k \in K\right\}$ for $1 \leq i \leq n-1$. We refer to Appendix D.4 for the proof.

Theorem 9 (Locality composition with randomness subset). Let $G_{k}$ for $k \in K$ be a set of fan-in 2 gadgets which all make an $\ell$-local use of a subset $\rho_{k}$ of their randomness. Consider the gadgets $G_{k}^{\prime}$ for $k \in K$ where the output of $G_{k}$ is locality refreshed with randoms $s_{i}^{(k)}$ for $1 \leq i \leq n-1$. Any composite gadget made of $G_{k}^{\prime}$ makes an $\ell$-local use of the randomness $\bigcup_{k \in K} \rho_{k}$, and for any $1 \leq i \leq n-1$, it makes a 3-local use of the randoms in $\left\{s_{i}^{(k)}: k \in K\right\}$.

For example if we compose a number of SecMultFLR gadgets, in the composite gadget the locality with respect to the randoms $r_{i j}^{(k)}$ for fixed $i, j$ is $\ell=1$, while the locality with respect to the randoms $s_{i}^{(k)}$ for fixed $i$ from the output locality refreshing is $\ell=3$. We stress that in the final implementation all the randomness (including the randomness from the locality refreshing) will be generated by the PRGs. Finally, we show in Appendix D.5 that the latter locality can be brought down to 1 ; for this it suffices to additionally perform a locality refreshing of the two inputs of each gadget, with independent sets of PRGs for the two inputs.

\subsection{First construction: multiple PRGs with SecMultFLR}

Our first construction is described in Figure 6. It consists in using the SecMult algorithm and perform a locality refresh after each gadget; this includes the SecMult gadget, the Xor gadget and the FullRefresh gadget. For every $1 \leq i<j \leq n$, an independent PRG generates all randoms $r_{i j}^{(k)}$ in the SecMult and FullRefresh gadgets. Similarly, for each $1 \leq i \leq n-1$, an independent PRG generates all randoms $s_{i}^{(k)}$ in all locality refreshing gadgets.

\section{Construction 1: multiple PRGs with SecMultFLR}

1. Given a circuit $C$, generate a private circuit $\left(I, C^{\prime}, O\right)$ with $n=t+1$ shares as follows: - replace every AND gate by the "double-SNI" gadget with SecMult and FullRefresh. Perform a locality refreshing LR after SecMult and FullRefresh.

- replace every XOR gate by the Xor gadget. Perform a locality refreshing LR after each Xor gadget.

2. Initialize $n(n-1) / 2$ PRG functions $G_{i j}$ for $1 \leq i<j \leq n$, each with $r$-wise independence parameter $r=t$.

3. Generate all randoms $r_{i j}^{(k)}$ in SecMult or FullRefresh gadget $k$ with the PRG function $G_{i j}$.

4. Initialize $n-1$ PRG functions $G_{i}^{\prime}$ for $1 \leq i<n$, each with $r$-wise independence parameter $r=3 t$.

5. Generate all randoms $s_{i}^{(k)}$ in the LR algorithm from gadget $k$ using the PRG function $G_{i}^{\prime}$.

Fig. 6. Private circuit construction with multiple PRGs with SecMultFLR.

From the locality composition theorem (Theorem 9), in the global construction the locality with respect to the randoms $\left\{r_{i j}^{(k)}: k \in K\right\}$ is $\ell_{r}=1$, while the locality with respect to the randoms $\left\{s_{i}^{(k)}: k \in K\right\}$ is $\ell_{s}=3$. From the PINI-R property of the gadgets and Theorem 8 , the full circuit is PINI-R. Therefore, from Proposition 4, it is secure in an extended model of security 
in which the adversary can get the previous randomness subsets with a single probe. From Lemma 11 , the PRGs for the $r_{i j}$ 's must be $t$-wise independent, while the PRGs for the $s_{i}$ 's must be $3 t$-wise independent. Since one requires $n(n-1) / 2$ independent PRGs for the $r_{i j}$ 's, and $n-1$ independent PRGs for the $s_{i}$ 's, the number of input randoms in the finite field is therefore, with $n=t+1$,

$$
n_{r}=n(n-1) / 2 \cdot t+(n-1) \cdot 3 t=\mathcal{O}\left(t^{3}\right) .
$$

Thus we have shown the following lemma. Compared to Lemma 9 for a single robust PRG with our SecMultILR algorithm, the randomness complexity is the same but the total running time goes down from $\tilde{\mathcal{O}}\left(s t^{5}\right)$ to $\tilde{\mathcal{O}}\left(s t^{3}\right)$.

Lemma 12 (multiple PRGs with SecMultFLR). Any function of circuit size $s$ admits a $t$ private implementation $(I, C, O)$ with the canonic encoder $I$ and decoder $O$, where $C$ uses $\mathcal{O}\left(t^{3}\right.$. $\log (s t))$ bits of randomness, and runs in time $\mathcal{O}\left(s \cdot t^{3} \cdot \log ^{2}(s t)\right)$.

\subsection{Second construction: multiple PRGs with SecMultILR}

Our second construction is described in Figure 7, based on the SecMultILR algorithm. As illustrated in Figure 8, a dedicated PRG generates the $r_{i j}$ 's for a given row $i$, in all gadgets. We first show that the SecMultILR algorithm makes a 1-local use of each row of randoms $r_{i j}$ and a 2-local use of each row of randoms $s_{i j}$; see Appendix D.6 for the proof.

Lemma 13 (Locality of SecMultILR). The SecMultILR algorithm makes a 1-local use of each randomness set $\rho_{i}=\left\{r_{i j}: i<j \leq n\right\}$ and a 2-local use of each randomness set $\rho_{i}^{\prime}=\left\{s_{i j}: i<j \leq\right.$ $n\}$.

\section{Construction 2: multiple PRGs with SecMultILR}

1. Given a circuit $C$, generate a private circuit $\left(I, C^{\prime}, O\right)$ with $n=t+1$ shares as follows: - replace every AND gate by the "double-SNI" gadget with SecMultILR and the corresponding FullRefreshILR. Perform a locality refreshing LR after each SecMultILR and FullRefreshILR.

- replace every XOR gate by the Xor gadget. Perform a locality refreshing LR after each Xor gadget.

2. Initialize $n-1$ PRG functions $G_{i}$ for $1 \leq i<n$, each with $r$-wise independence parameter $r=t$.

3. Generate all randoms $r_{i j}^{(k)}$ in SecMultILR or FullRefreshILR gadget $k$ with the PRG function $G_{i}$

4. Initialize $n-1 \mathrm{PRG}$ functions $G_{i}^{\prime}$ for $1 \leq i<n$, each with $r$-wise independence parameter $r=2 t$.

5. Generate all randoms $s_{i j}^{(k)}$ in SecMultILR or FullRefreshILR gadget $k$ using the PRG function $G_{i}^{\prime}$

6. Initialize $n-1$ PRG functions $G_{i}^{\prime \prime}$ for $1 \leq i<n$, each with $r$-wise independence parameter $r=3 t$.

7. Generate all randoms $s_{i}^{(k)}$ in the LR algorithm using the PRG function $G_{i}^{\prime \prime}$.

Fig. 7. Private circuit construction with multiple PRGs with SecMultILR.

From Lemma 13 and Theorem 9, in the global construction the locality with respect to the subsets of randoms $\rho_{i}=\left\{r_{i j}^{(k)}: i<j \leq n, k \in K\right\}$ is equal to 1 , the locality with respect to the subsets of randoms $\rho_{i}^{\prime}=\left\{s_{i j}^{(k)}: i<j \leq n, k \in K\right\}$ is equal to 2 , and the locality with respect to the subsets of randoms $\rho_{i}^{\prime \prime}=\left\{s_{i}^{(k)}: k \in K\right\}$ is still equal to 3 , for each $1 \leq i<n$. As previously, from the PINI-R property of the gadgets and Proposition 8, the full circuit is PINI-R. Therefore, it is secure in an extended model of security in which the adversary can get the previous randomness 
subsets with a single probe. From Lemma 11, the corresponding PRGs must therefore have $r$-wise independence parameter $r=t, r=2 t$ and $r=3 t$ respectively. The main difference is that now there are only $n-1$ independent PRGs to generate the $r_{i j}^{(k)}$ (instead of $n(n-1) / 2$ previously), because a given PRG generates those randoms for all indices $j$. The total number of input randoms in the finite field is therefore

$$
n_{r}=(n-1) \cdot t+(n-1) \cdot 2 t+(n-1) \cdot 3 t=\mathcal{O}\left(t^{2}\right) .
$$

Thus we have shown the following lemma. Asymptotically this is the most efficient technique (see Table 1 for a comparison), and also the most efficient in practice (see the next section for our implementation results on AES).

Lemma 14 (multiple PRGs with SecMultILR). Any function of circuit size $s$ admits a t-private implementation $(I, C, O)$ with the canonic encoder $I$ and decoder $O$, where $C$ uses $\mathcal{O}\left(t^{2} \cdot \log (s t)\right)$ bits of randomness, and runs in time $\mathcal{O}\left(s \cdot t^{3} \cdot \log ^{2}(s t)\right)$.

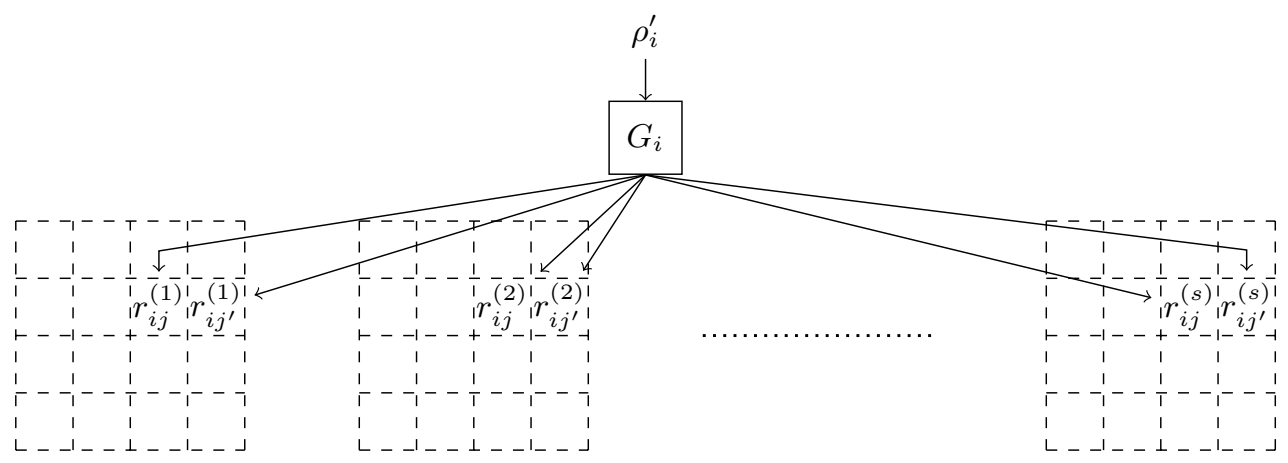

Fig. 8. In Construction 2, a dedicated PRG generates the $r_{i j}$ 's for a given row $i$ in all gadgets, from a random seed $\rho_{i}^{\prime}$.

\section{Application to AES}

In this section we describe a concrete implementation of our techniques for AES; the goal is to minimize the total amount of randomness used to protect AES against $t$-th order attack. We provide the source code in $\mathrm{C}$ in [Cor19b].

\subsection{The AES circuit and the Rivain-Prouff countermeasure}

To implement the AES SBox, we need to perform 4 multiplications, and 2 mask refreshing per byte; see [RP10] for the sequence of operations. For the mask refreshing, we use the multiplication based refreshing FullRefresh recalled in Appendix B.2. We refer to $\left[\mathrm{BBD}^{+} 16\right]$ for the proof that the $x^{254}$ gadget is $(n-1)$-SNI; this implies that the gadget is PINI. Thus, this amounts to performing 6 multiplications per byte. Since there are 16 bytes to process per round, the number of required multiplications is $6 \times 16=96$ per round. Thus for the 10 rounds of the AES, one will perform $96 \times 10=960$ multiplications.

\subsection{Implementation with single robust PRG}

We first consider an implementation with a single robust PRG as in Section 3, with 3 possible algorithms: the original $\left[\mathrm{IKL}^{+} 13\right]$ construction with a locality refresh after each multiplication gadget (SecMultFLR), and our new SecMultILR and SecMultILR2 algorithms. For those three algorithms, 
we provide in Table 7 the total number of pseudo-randoms to be generated for the AES circuit, the corresponding locality parameter $\ell$, and the number of 8-bit randoms from the TRNG to generate the seed of the PRG, as a function of the number of shares $n$, for security against $t$ probes with $n=t+1$.

\begin{tabular}{|l|c|c|c|c|}
\cline { 2 - 5 } \multicolumn{1}{c|}{} & SecMult [RP10] & SecMultFLR $\left[\mathrm{IKL}^{+} 13\right]$ & SecMultILR & SecMultILR2 \\
\hline Mult & $480 n(n-1)$ & $(480 n+960)(n-1)$ & $960 n(n-1)$ & $(480 n+960)(n-1)$ \\
\hline Xor & - & \multicolumn{3}{|c|}{$160(n-1)$} \\
\hline Pseudo-rand & - & $(480 n+1120)(n-1)$ & $(960 n+160)(n-1)$ & $(480 n+1120)(n-1)$ \\
\hline \hline Locality $\ell$ & - & $\begin{array}{c}\max (4(n-1), \\
\left.n^{2} / 4+5 n / 2-c\right)\end{array}$ & $4(n-1)$ & $4(n-1)$ \\
\hline True-rand & $480 n(n-1)$ & $\begin{array}{c}2 n(n-1) \cdot \max (4(n-1), \\
\left.n^{2} / 4+5 n / 2-c\right)\end{array}$ & $8 n(n-1)^{2}$ & $8 n(n-1)^{2}$ \\
\hline
\end{tabular}

Table 7. For AES, total number of pseudo-randoms and number of 8-bit TRNG calls, for a single robust PRG, as a function of the number of shares $n$. We have $c=3$ for even $n$, and $c=11 / 4$ for odd $n$. We assume that $n \leq 12$.

We now explain the content of Table 7. For each of the 3 algorithms, the number of pseudorandoms is the number of randoms from Table 3 in Section 3, multiplied by 960, since one must perform 960 multiplications. Furthermore, the MixColumns operation requires 48 xors. Normally we should perform a locality refresh after each xor, but in the particular case of the AES, we can do the locality refresh only after the 3 xors of the MixColumns for each byte. In that case, the locality parameter with respect to MixColumns is then $4(n-1)$, instead of $2(n-1)$ for a single xor. The locality of the global circuit is then the max of locality parameter $\ell$ from Table 3 and $4(n-1)$. Equivalently, we can perform such locality refresh as input of the SubByte operation, which enables to keep the MixColumns unmodified. For the MixColumns, one therefore needs to perform 16 locality refresh per round, which gives a total of 160 locality refresh for the 10 rounds of the AES, which requires 160(n-1) pseudo-randoms. Finally, we assume that the round keys are already masked without PRG, and so we don't need to perform a locality refreshing after the AddRoundKey.

Let $m$ the total number of pseudo-randoms over $\mathbb{F}_{2^{8}}$ that must be generated. To determine the finite field $\mathbb{F}=\mathbb{F}_{2^{8 k}}$ used by the PRG, we must ensure $m \leq k \cdot\left|\mathbb{F}_{2^{8 k}}\right|=k \cdot 2^{8 k}$. Namely a single polynomial evaluation over $\mathbb{F}_{2^{8 k}}$ generates $k$ bytes of pseudo-random. One must then use a PRG with $r$-wise independence parameter $r=\ell \cdot(n-1)$. Using the trivial construction with the xor of $n=t+1$ polynomial evaluations (to provide resistance against $t$ probes), the total number of fresh random values over $\mathbb{F}_{2^{8}}$ is then $n_{r}=k \cdot n \cdot r=k \cdot n(n-1) \cdot \ell$.

For the three algorithms one can work over $\mathbb{F}_{2^{16}}$ for $n \leq 12$; therefore for simplicity we take $k=2$ in Table 7. For SecMultILR and SecMultILR2, the total number of TRNG calls over $\mathbb{F}_{2^{8}}$ is then $n_{r}=k \cdot n(n-1) \cdot 4(n-1)=4 k \cdot n(n-1)^{2}$ with $k=2$ for $n \leq 12$, and $k=3$ for $13 \leq n \leq 229$, instead of $480 n(n-1)$ for the original Rivain-Prouff countermeasure; therefore one needs fewer TRNG calls than Rivain-Prouff for $n \leq 40$. We summarize in Table 9 below the number of input random bytes required for AES for small values of $n$, compared with the original Rivain-Prouff countermeasure.

\subsection{Implementation with multiple PRGs}

We now consider an implementation of AES with multiple PRGs, as in Section 4. We consider the SecMultFLR algorithm corresponding to Construction 1, and the SecMultILR algorithm corresponding to Construction 2. As previously, we provide in Table 8 the total number of pseudo-randoms to be generated for the AES circuit, and the number of 8-bit randoms from the TRNG.

As previously, we only perform a locality refresh after the 3 xors of the MixColumns (equivalently, before each SubByte). Moreover we don't perform the LR algorithm after SecMultILR as in 


\begin{tabular}{|l|c|c|c|}
\cline { 2 - 4 } \multicolumn{1}{c|}{} & SecMult $[\mathrm{RP} 10]$ & SecMultFLR & SecMultILR \\
\hline Pseudo-rand & - & $(480 n+1120)(n-1)$ & $(960 n+160)(n-1)$ \\
\hline \hline Locality $\ell_{r}$ of $r_{i j}$ & - & 1 & 1 \\
\hline Number of PRGs $\left(r_{i j}\right)$ & - & $n(n-1) / 2$ & $n-1$ \\
\hline True-rand per PRG $\left(r_{i j}\right)$ & - & $2(n-1)$ & $2(n-1)$ \\
\hline \hline Locality $\ell_{s}$ of $s_{i j}$ and $s_{i}$ & - & 5 & 5 \\
\hline Number of PRGs $\left(s_{i}\right.$ and $\left.s_{i j}\right)$ & - & $n-1$ & $n-1$ \\
\hline True-rand per PRG $\left(s_{i j}\right.$ and $\left.s_{i}\right)$ & - & $10(n-1)$ & $10(n-1)$ \\
\hline \hline Total True-Rand & $480 n(n-1)$ & $(n+10)(n-1)^{2}$ & $12(n-1)^{2}$ \\
\hline
\end{tabular}

Table 8. For AES, total number of Pseudo-random and True-random values to generate with the multiple PRGs approach, as a function of the number of shares $n$. Values for the Rivain-Prouff countermeasure are also recalled for comparison.

Construction 2, since the output of SecMultILR is already locality refreshed. Therefore the number of pseudo-randoms is the same as in the previous section. We use two classes of independent PRGs. The first class of independent PRGs is used to generate the $r_{i j}$ 's from SecMultFLR and SecMultILR algorithms, with locality $\ell_{r}=1$; therefore the PRGs must be $\ell_{r} t$-wise independent. We need $n(n-1) / 2$ such PRGs for SecMultFLR, and only $n-1$ for SecMultILR. Working over $\mathbb{F}_{2^{16}}$, each PRG requires $2 \ell_{r} t=2(n-1)$ random bytes. Similarly, the second class of PRGs is used to generate randoms $s_{i}$ from the locality refresh, and also the randoms $s_{i j}$ for the internal locality refresh in SecMultILR, with locality $\ell_{s}=5$. Namely we only perform the locality refresh after the 3 xors of the MixColumns, and therefore the locality is $\ell_{s}=5$ (instead of $\ell_{s}=3$ ). Note that for SecMultILR we can use the same class of PRGs to generate the randoms $s_{i j}$ 's from SecMultILR and the randoms $s_{i}$ 's from LR, instead of two classes in Construction 2 from Section 4; namely it is easy to see that the locality with respect to the corresponding randomness subsets is still equal to 5. Therefore the PRGs must be $\ell_{s} t$-wise independent; working over $\mathbb{F}_{2^{16}}$, each PRG requires 10( $n-1)$ bytes of TRNG.

In summary, for SecMultFLR, the total number of 8-bit TRNG calls is therefore

$$
n_{r}=n(n-1) / 2 \cdot 2(n-1)+(n-1) \cdot 10(n-1)=(n+10)(n-1)^{2}
$$

and for SecMultILR, we get

$$
n_{r}=(n-1) \cdot 2(n-1)+(n-1) \cdot 10(n-1)=12(n-1)^{2}
$$

instead of $480 n(n-1)$ in the original Rivain-Prouff countermeasure. ${ }^{3}$

A simple 3-wise independent PRG. Finally, we consider the simple 3-wise independent PRG from Section 2.3:

$$
G\left(x_{1}, \ldots, x_{d}, y_{1}, \ldots, y_{d}\right)=\left(x_{i} \oplus y_{j}\right)_{1 \leq i, j \leq d}
$$

Since the PRG function $G$ expands from $2 d$ to $d^{2}$ bits (or bytes), the number of input randoms becomes $\mathcal{O}(\sqrt{s})$ instead of $\mathcal{O}(s)$, where $s$ is the circuit size. Note that this is worse than the polynomial-based PRG used previously that requires only $\mathcal{O}(\log s)$ randoms, but the above function $G$ is very fast since generating a pseudo-random only takes a single xor.

Since the above PRG only achieves 3-wise independence, we want to minimize the locality. Therefore, we perform a locality refresh of the 2 inputs of each gadget (with two distinct sets of independent PRGs), and we perform a locality refresh of the outputs of each gadget (SecMult, Xor and FullRefresh), using another distinct set of independent PRGs. As shown in Appendix D.5, the locality with respect to each subset of randoms is then always $\ell=1$; therefore, we can use a PRG

\footnotetext{
${ }^{3}$ For the SecMultILR algorithm, a PRG for the $s_{i j}$ and $s_{i}$ must generate a maximum of $960(n-1)+160$ pseudorandoms; therefore one can work over $\mathbb{F}_{2^{16}}$ as long as $n \leq 136$.
} 
with $r$-wise independence $r=t=n-1$. This implies that this specific PRG only works for $n=3$ and $n=4$ shares. We argue in Appendix E.2 that the total number of input bytes for AES is 642 for $n=3$ and 1056 for $n=4$, instead of 2880 and 5760 respectively for the original Rivain-Prouff countermeasure.

\begin{tabular}{|l|c|c|c|c|c|c|c|}
\cline { 3 - 7 } \multicolumn{1}{c|}{} & \multicolumn{3}{c|}{ Single robust PRG } & \multicolumn{3}{c|}{ Multiple PRGs } \\
\cline { 2 - 8 } \multicolumn{1}{c|}{} & {$[$ RP10] } & SecMultFLR & SecMultILR & SecMultILR2 & SecMultFLR & SecMultILR & 3 -wise SecMultFLR \\
\hline$n=3$ & 2880 & 96 & 96 & 96 & 52 & 48 & 642 \\
\hline$n=4$ & 5760 & 288 & 288 & 288 & 126 & 108 & 1056 \\
\hline$n=5$ & 9600 & 640 & 640 & 640 & 240 & 192 & - \\
\hline$n=6$ & 14400 & 1260 & 1200 & 1200 & 400 & 300 & - \\
\hline$n=7$ & 20160 & 2268 & 2016 & 2016 & 612 & 432 & - \\
\hline$n=8$ & 26880 & 3696 & 3136 & 3136 & 882 & 588 & - \\
\hline$n=9$ & 34560 & 5760 & 4608 & 4608 & 1216 & 768 & - \\
\hline$n=10$ & 43200 & 8460 & 6480 & 6480 & 1620 & 972 & - \\
\hline
\end{tabular}

Table 9. For AES, total number of TRNG bytes to generate for single and multiple PRGs methods, depending of the number of shares $n$. We also provide the number of TRNG bytes for the original Rivain-Prouff countermeasure.

Summary. We summarize in Table 9 the number of input random bytes required for AES for all previous methods, as a function of the number of shares $n$, in order to achieve $t$-th order security, with $t=n-1$. We see that the most efficient method (in terms of minimizing the number of TRNG calls) is the SecMultILR algorithm with multiple PRGs. Namely for small values of $t$ we obtain almost two orders of magnitude improvement compared to the original Rivain-Prouff countermeasure.

\subsection{Concrete Implementation}

We have implemented our constructions for AES in C, on a $44 \mathrm{MHz}$ ARM-Cortex M3 processor. The processor is used in a wide variety of products such as passports, bank cards, SIM cards, secure elements, etc. The embedded TRNG module can run in parallel of the CPU, but it is relatively slow: according to our measurements on emulator, it outputs 32 bits of random in approximately 6000 cycles. Our results, obtained by running the code on emulator, are given in Table 10, and are compared with the classical Rivain-Prouff countermeasure.

We see that the most efficient countermeasure is the SecMultFLR algorithm with multiple PRGs, using the 3 -wise independent PRG. For $n=3$ and $n=4$ we obtain a $52 \%$ and $61 \%$ speedup respectively, compared to Rivain-Prouff. We provide the source code in [Cor19b].

\section{References}

[AIS18] Prabhanjan Ananth, Yuval Ishai, and Amit Sahai. Private circuits: A modular approach. In Advances in Cryptology - CRYPTO 2018 - Proceedings, Part III, pages 427-455, 2018. Final version available at https://eprint.iacr.org/2018/566.pdf.

$\left[\mathrm{BBD}^{+} 15\right]$ Gilles Barthe, Sonia Belaïd, François Dupressoir, Pierre-Alain Fouque, Benjamin Grégoire, and PierreYves Strub. Verified proofs of higher-order masking. In Advances in Cryptology - EUROCRYPT 2015 Proceedings, Part I, pages 457-485, 2015.

$\left[\mathrm{BBD}^{+} 16\right]$ Gilles Barthe, Sonia Belaïd, François Dupressoir, Pierre-Alain Fouque, Benjamin Grégoire, Pierre-Yves Strub, and Rébecca Zucchini. Strong non-interference and type-directed higher-order masking. In Proceedings of the 2016 ACM SIGSAC Conference on Computer and Communications Security, Vienna, Austria, October 24-28, 2016, pages 116-129, 2016. Publicly available at https://eprint.iacr.org/ 2015/506.pdf.

$\left[\mathrm{BBP}^{+} 16\right]$ Sonia Belaïd, Fabrice Benhamouda, Alain Passelègue, Emmanuel Prouff, Adrian Thillard, and Damien Vergnaud. Randomness complexity of private circuits for multiplication. In Advances in Cryptology EUROCRYPT 2016 - Proceedings, Part II, pages 616-648, 2016. 


\begin{tabular}{|c|c|c|c|c|c|c|c|c|}
\hline & & \multicolumn{3}{|c|}{ Single robust PRG } & \multicolumn{3}{|c|}{ Multiple PRGs } \\
\hline & & [RP10] & SecMultFLR & SecMultILR & SecMultILR2 & SecMultFLR & SecMultILR & 3-wise SecMultFLR \\
\hline \multirow{2}{*}{$n=3$} & Mcycles & 20.6 & 65.6 & 76.8 & 65.4 & 12 & 14.1 & 9.8 \\
\hline & ratio & 1 & 3.18 & 3.73 & 3.17 & 0.58 & 0.68 & 0.48 \\
\hline \multirow{2}{*}{$n=4$} & Mcycles & 40.2 & 235.1 & 425.1 & 324.9 & 24.6 & 34.7 & 15.5 \\
\hline & ratio & 1 & 5.85 & 10.57 & 8.08 & 0.61 & 0.86 & 0.39 \\
\hline \multirow{2}{*}{$n=5$} & Mcycles & "65.8 & 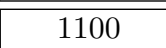 & $\overline{1541.5}$ & $\overline{1097.1}$ & 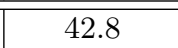 & $\overline{70}$ & - \\
\hline & ratio & 1 & 16.72 & 23.43 & 16.67 & 0.65 & 1.06 & - \\
\hline \multirow{2}{*}{$n=6$} & Mcycles & 97.5 & 3042.1 & 4278.3 & 2898.5 & 67.2 & 124.1 & - \\
\hline & ratio & 1 & 31.20 & 43.88 & 29.73 & 0.69 & 1.27 & - \\
\hline
\end{tabular}

Table 10. Smart-card implementation results, on a $44 \mathrm{MHz}$ ARM-Cortex M3 processor, with an embedded TRNG module. We provide the timings in millions of clock cycles, and the ratio with respect to the Rivain-Prouff countermeasure.

[Cor18] Jean-Sébastien Coron. Formal verification of side-channel countermeasures via elementary circuit transformations. In Applied Cryptography and Network Security ACNS, volume 10892, pages 65-82, 2018.

[Cor19a] Jean-Sébastien Coron. CheckMasks: formal verification of side-channel countermeasures, 2019. Publicly available at https://github.com/coron/checkmasks.

[Cor19b] Jean-Sébastien Coron. Implementation of higher-order countermeasures, 2019. Publicly available at https://github.com/coron/htable/.

[CS18] Gaëtan Cassiers and François-Xavier Standaert. Trivially and efficiently composing masked gadgets with probe isolating non-interference. Cryptology ePrint Archive, Report 2018/438, 2018. https://eprint. iacr.org/2018/438.

[DDF14] Alexandre Duc, Stefan Dziembowski, and Sebastian Faust. Unifying leakage models: From probing attacks to noisy leakage. In Advances in Cryptology - EUROCRYPT 2014 - Proceedings, pages 423-440, 2014.

[FPS17] Sebastian Faust, Clara Paglialonga, and Tobias Schneider. Amortizing randomness complexity in private circuits. In Advances in Cryptology - ASIACRYPT 2017 - Proceedings, Part I, pages 781-810, 2017.

[GUV09] Venkatesan Guruswami, Christopher Umans, and Salil P. Vadhan. Unbalanced expanders and randomness extractors from parvaresh-vardy codes. J. ACM, 56(4):20:1-20:34, 2009.

$\left[\mathrm{IKL}^{+} 13\right]$ Yuval Ishai, Eyal Kushilevitz, Xin Li, Rafail Ostrovsky, Manoj Prabhakaran, Amit Sahai, and David Zuckerman. Robust pseudorandom generators. In ICALP 2013, Proceedings, Part I, pages 576-588, 2013.

[ISW03] Yuval Ishai, Amit Sahai, and David Wagner. Private circuits: Securing hardware against probing attacks. In Advances in Cryptology - CRYPTO 2003, Proceedings, pages 463-481, 2003.

[OMHT06] Elisabeth Oswald, Stefan Mangard, Christoph Herbst, and Stefan Tillich. Practical second-order DPA attacks for masked smart card implementations of block ciphers. In $C T$ - $R S A$, pages 192-207, 2006.

[RP10] Matthieu Rivain and Emmanuel Prouff. Provably secure higher-order masking of AES. In Cryptographic Hardware and Embedded Systems, CHES 2010, Proceedings, pages 413-427, 2010.

\section{A PRG based on bipartite expander graph}

\section{A.1 Definitions}

Bipartite graph and PRG. We note by $[n]$ the set of integers between 1 and $n$. A bipartite graph is a triple $([m],[n], E)$ where $[m]$ is the set of left vertices, $[n]$ of right vertices, and $E \subset[m] \times[n]$ is a set of edges connecting the left vertices with the right vertices. Given any subset $V \subseteq[\mathrm{m}]$, we denote by $\Gamma(V)$ the right neighbors of $V$; formally $\Gamma(V)=\{v \in[n]: \exists u \in[m]$ such that $(u, v) \in E\}$.

We recall the simple construction of a PRG from bipartite graph $\left[\mathrm{IKL}^{+} 13\right]$. A bipartite graph $H=([m],[n], E)$ induces a linear function $G_{H}:\{0,1\}^{n} \rightarrow\{0,1\}^{m}$ where the $i$-th output bit for $1 \leq i \leq m$ is the parity of the input bits corresponding to the neighbors of $i$. Formally, $G_{H}\left(x_{1}, \ldots, x_{n}\right)=\left(y_{1}, \ldots, y_{m}\right)$ where $y_{i}=\oplus_{j \in \Gamma(i)} x_{j}$. In other words, every left vertex gives an output bit, computed as the xor of the input bits corresponding to the right vertices to which it is connected. See Figure 9 for an example of a simple bipartite graph, with the corresponding PRG.

Expander graphs. Letting $V \subset[m]$ on the left, a vertex $v \in[n]$ on the right is said to be a unique neighbor of $V$ if there is a single edge from $V$ to $v$. We let $U(V)$ denote the collection of unique neighbors of $V$. We say that a bipartite graph $([m],[n], E)$ is a $\ell$-unique neighbor expander if any set $V \subset[m]$ on the left with $|V| \leq \ell$ has a unique neighbor. 


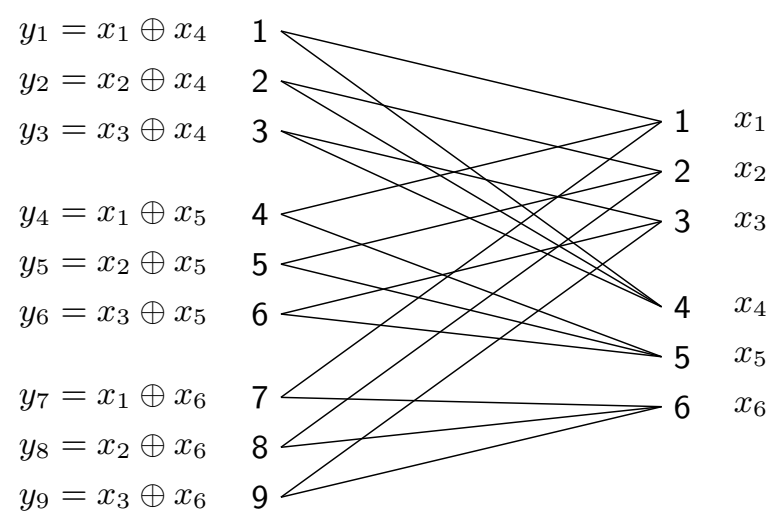

Fig. 9. Example of a $([m],[n], E)$ bipartite graph with $m=9$ and $n=6$, with the corresponding PRG. The graph is a $(2,3 / 2)$-expander.

Lemma 15. If a bipartite graph $H=([m],[n], E)$ is a $\ell$-unique neighbor expander, then the corresponding $P R G G_{H}$ is $\ell$-wise independent.

Proof. We consider the $m \times n$ matrix of row vectors corresponding to the linear function $G_{H}\left(x_{1}, \ldots\right.$ ,$\left.x_{n}\right)$. We show that any subset of at most $\ell$ rows are linearly independent. Namely a linear combination of at most $\ell$ rows cannot be equal to 0 , since by the $\ell$-unique neighbor expander property, there is an input $x_{i}$ that must appear in a single row. Since any subset of at most $\ell$ rows are linearly independent, the PRG $G_{H}$ is $\ell$-wise independent.

Expander graphs. To construct bipartite graphs with the unique neighbor property, we will use expander graphs.

Definition 11. A bipartite graph $([m],[n], E)$ with left vertices $[m]$ and right vertices $[n]$ is an $(\ell, b)$-expander if for any subset $V \subseteq[m]$ on the left with $|V| \leq \ell$, we have that $|\Gamma(V)| \geq b|V|$.

The following lemma shows that if the expansion factor $b$ is such that $b>d / 2$, then any set $V$ such that $|V| \leq \ell$ has a unique neighbor; therefore the corresponding PRG will be $\ell$-wise independent.

Lemma 16. Consider an $(\ell, b)$-expander graph $([m],[n], E)$ of degree $d$. For any $V \subset[m]$ with $|V| \leq \ell$

$$
d|V| \geq|\Gamma(V)| \geq|U(V)| \geq(2 b-d)|V|
$$

Proof. The number of edges out of $V$ is $d|V|$, so $d|V| \geq|\Gamma(V)|$. Out of these $d|V|$ edges, due to the expansion property of the graph, at least $b|V|$ go to distinct vertices; there remains $(d-b)|V|$ edges, which can eliminate the uniqueness of at most $(d-b)|V|$ vertices in $\Gamma(V)$. Therefore $|U(V)| \geq$ $b|V|-(d-b)|V| \geq(2 b-d)|V|$.

\section{A.2 A simplified proof of strong robustness for PRG}

In this section we provide a simplified proof of strong robustness of PRG based on expander graph.

Theorem $10\left(\left[\mathbf{I K L}^{+} \mathbf{1 3}\right]\right)$. Suppose $H$ is a d-left-regular $(\ell, d / 2+c)$-expander. Then $G_{H}$ is a strong $(\ell, c \ell / d, d / c)$-robust $P R G$.

The proof of Theorem 10 is based on the lemma below, showing that an expander graph is robust against the removal of vertices; that is, even if we remove some vertices on the right, the induced graph remains an expander graph. More precisely, the following lemma is a slightly stronger variant of $\left[\mathrm{IKL}^{+} 13\right.$, Lemma 6], which was used to prove the PRG weak robustness only. 
The difference is that in our lemma the induced graph has right vertices $[n] \backslash(S \cup \Gamma(T))$ instead of only $[n] \backslash S$; that is, we remove more vertices on the right. Another difference is that for the resulting graph we only get the $\ell$-unique neighbor property (instead of the expansion property), since this is only what we need. The rest of the proof is essentially the same as in $\left[\mathrm{IKL}^{+} 13\right]$.

Lemma 17. Suppose $H=([m],[n], E)$ is a d-left-regular $(\ell, d / 2+c)$-expander. Then for any $S \subseteq[n]$ on the right of size $|S| \leq c \ell$, there exists $T \subseteq[m]$ on the left with $|T| \leq|S| / c$ such that the induced graph on left vertices $[m] \backslash T$ and right vertices $[n] \backslash(S \cup \Gamma(T))$ is a $(\ell-|T|)$-unique neighbor expander.

Proof. We first construct a subset $T$ on the left with a large fraction of its right neighbors $\Gamma(T)$ in $S$. We let $T$ be a subset on the left that has the maximum size among all subsets $T^{\prime}$ on the left with size at most $\ell$ such that $\left|\Gamma\left(T^{\prime}\right) \backslash S\right| \leq d / 2 \cdot\left|T^{\prime}\right|$; the set $T$ is well defined because $T^{\prime}=\emptyset$ is such a subset. We have by definition of $T$ :

$$
|\Gamma(T)|=|\Gamma(T) \backslash S|+|\Gamma(T) \cap S| \leq d / 2 \cdot|T|+|\Gamma(T) \cap S|
$$

From the expansion property of $H$ and $|T| \leq \ell$, we must have:

$$
|\Gamma(T)| \geq(d / 2+c)|T|
$$

Combining the two inequalities, we get:

$$
|\Gamma(\mathrm{T}) \cap S| \geq|\Gamma(T)|-d / 2 \cdot|T| \geq c \cdot|T|
$$

which shows that $T$ has a large fraction of neighbors in $S$. We obtain $|T| \leq|\Gamma(\mathrm{T}) \cap S| / c \leq|S| / c$ as required.

We now consider any non-empty subset $V$ on the left with $V \cap T=\emptyset$ and $|V| \leq \ell-|T|$. We show that $V$ must have many neighbors outside the set $S \cup \Gamma(T)$. Namely $T \cup V$ has many neighbors by the expansion property, and the neighbors of $T$ are fairly concentrated in $S$. More precisely, since $|T \cup V|=|T|+|V| \leq \ell$ and $T$ was selected as a subset of maximal size with size at most $\ell$ such that $|\Gamma(T) \backslash S| \leq d / 2 \cdot|T|$, we must have:

$$
|\Gamma(T \cup V) \backslash S|>d / 2 \cdot|T \cup V|=d / 2 \cdot(|T|+|V|)
$$

Moreover we have:

$$
|\Gamma(T \cup V) \backslash S|=|(\Gamma(T) \cup \Gamma(V)) \backslash S|=|\Gamma(T) \backslash S|+|\Gamma(V) \backslash(\Gamma(T) \cup S)|
$$

Using $|\Gamma(T) \backslash S| \leq d / 2 \cdot|T|$, we obtain:

$$
\begin{aligned}
|\Gamma(V) \backslash(\Gamma(T) \cup S)| & =|\Gamma(T \cup V) \backslash S|-|\Gamma(T) \backslash S| \\
& >d / 2 \cdot(|T|+|V|)-d / 2 \cdot|T| \\
& >d / 2 \cdot|V|
\end{aligned}
$$

This shows that the induced graph on left vertices $[m] \backslash T$ and right vertices $[n] \backslash(S \cup \Gamma(T))$ is a $(\ell-|T|)$-unique neighbor expander.

Proof (of theorem 10). Let $t \leq k$ be the number of probes in the circuit, with $k=c \ell / d$. Let $S$ be the vertices on the right that are affected by the probes. Since every output is the sum of exactly $d$ randoms, we must have $|S| \leq t d \leq k d=c \ell$. Therefore we can apply Lemma 17, and there exists a set $T$ on the left with $|T| \leq|S| / c \leq t d / c$ such that the induced graph on left vertices $[m] \backslash T$ and right vertices $[n] \backslash(S \cup \Gamma(T))$ is a $(\ell-|T|)$-unique neighbor expander. We let $S^{\prime}=S \cup \Gamma(T)$. Therefore, conditioned on any fixing of the input bits in $S^{\prime}$, the input bits in $S$ are fixed and the output bits in $T$ are fixed (because the input bits in $\Gamma(T)$ are fixed), and the output bits that are not in $T$ are $r$-wise independent, where $r=\ell-|T| \geq \ell-t d / c$, which gives $q=d / c$. 


\section{A.3 The bipartite expander graph construction from [GUV09]}

In this section we recall the bipartite expander graph construction from [GUV09], and its application to PRG. We fix the field $\mathbb{F}_{q}$. Let $E(Y)$ be an irreducible polynomial of degree $n$ over $\mathbb{F}_{q}$. We identity elements of $\mathbb{F}_{q}^{n}$ with univariate polynomials over $\mathbb{F}_{q}$ with degree at most $n-1$. Let $h$ be an integer parameter. Let $d \leq q$. The expander is the bipartite graph $\Gamma: \mathbb{F}_{q}^{n} \times[d] \rightarrow[d] \times \mathbb{F}_{q}^{m}$ defined as:

$$
\Gamma(f, i) \stackrel{\text { def }}{=}\left[i, f(i),\left(f^{h} \bmod E\right)(i),\left(f^{h^{2}} \bmod E\right)(i), \ldots,\left(f^{h^{m-1}} \bmod E\right)(i)\right]
$$

Theorem 11 ([GUV09]). The graph $\Gamma: \mathbb{F}_{q}^{n} \times[d] \rightarrow[d] \times \mathbb{F}_{q}^{m}$ defined above is a $\left(h^{m}, A\right)$ expander for $A=d-(n-1)(h-1) m$.

We note that we can obtain a better bound for $m=1$, which enables to prove $d$-wise independence for a bipartite graph construction of degree $d$.

Lemma 18. The graph $\Gamma: \mathbb{F}_{q}^{n} \times[d] \rightarrow[d] \times \mathbb{F}_{q}$ defined above with $m=1$ is a $(h, A)$ expander for $A=d-(n-1)(h-1) / 2$. Taking $n=2, G_{\Gamma}$ is a d-wise independent PRG.

Proof. Let $V$ be a list of distinct polynomials $\left(f_{1}, \ldots, f_{|V|}\right)$. Given two polynomials $f_{j}$ and $f_{k}$ for $j \neq k$, we must have $\left|\Gamma\left(f_{j}\right) \cap \Gamma\left(f_{k}\right)\right| \leq n-1$, since otherwise the two polynomials $f_{j}$ and $f_{k}$ of degree at most $n-1$ would take the same value on $n$ distinct points and would be identical. This implies:

$$
|\Gamma(T)| \geq \sum_{i=1}^{|T|}(d-(i-1) \cdot(n-1)) \geq d|T|-(n-1) \frac{(|T|-1)|T|}{2}
$$

For $|T| \leq h$, we obtain $|\Gamma(T)| \geq|T| \cdot(d-(n-1)(h-1) / 2)$ as required.

Finally, taking $h=d$ and $n=2$, we get that $\Gamma$ is a $(d, d / 2+1 / 2)$-expander. Therefore it is a $d$-unique neighbor expander, and the corresponding $G_{\Gamma}$ is a $d$-wise independent PRG.

We note that the 3-wise independent PRG construction of Section 2.3 corresponds to $n=2$ and $d=2$. We fix two distinct elements $a$ and $b$ of $\mathbb{F}_{q}$. Given a polynomial $f$ of degree at most 1 on the left, the polynomial $f$ has two neighbors $(1, f(a))$ and $(2, f(b))$ on the right. Since there are $q^{2}$ polynomials $f$ in $\mathbb{F}_{q}$ of degree at most 1 , the expander graph $\Gamma$ has $q^{2}$ input vertices and $2 q$ output vertices. Given any two right vertices $(1, x)$ and $(2, y)$ for $x, y \in \mathbb{F}_{q}$, there is a unique $f \in \mathbb{F}_{q}^{2}$ on the left such that the two neighbors of $f$ are $(1, x)$ and $(2, y)$. In the corresponding PRG $G_{\Gamma}$, each of the $q^{2}$ bits of output is therefore the xor of two bits, each taken from the two input lists of $q$ bits.

Theorem 12 ([IKL $\left.\left.{ }^{+} \mathbf{1 3}\right]\right)$. For any $\eta>0$, there exists $\delta, C, \tilde{n}_{0}>0$ such that for any $\tilde{n} \geq \tilde{n}_{0}$, there is an explicit d-local strong $\left(\tilde{n}^{1-\eta}, \tilde{n}^{1-\eta}, 4\right)$-robust independent $P R G G:\{0,1\}^{\tilde{n}} \rightarrow\{0,1\}^{\tilde{m}}$ for $\tilde{m}=\exp \left(\tilde{n}^{\delta}\right)$, with $d \leq \log ^{C} \tilde{m}$.

Proof. We recall the parameters of the bipartite graph and the corresponding PRG in Table 11. The number of left vertices must be greater than the PRG output size, which gives the condition $q^{n} \geq \tilde{m}$. Similarly the PRG input size must be greater than the number of right vertices, which gives $\tilde{n} \geq q^{m+1}$. The maximum set size $\ell=h^{m}$ of the bipartite expander graph must be greater than the desired $r$-wise independence of the PRG, which gives the condition $h^{m} \geq \tilde{n}^{1-\eta}$. In the bipartite expander graph from [GUV09], the expansion factor is $A=d-(n-1)(h-1) m$. Writing $A=d / 2+c$, this gives $c=d / 2-(n-1)(h-1) m$. From Theorem 10 , we must have $d / c \leq 4$ and $\ell \cdot c / d \geq \tilde{n}^{1-\eta}$. This gives $c \geq d / 4$, which requires $(n-1)(h-1) m \leq d / 4$. We take $q=d$.

We obtain the following conditions:

$$
\begin{aligned}
q^{n} & \geq \tilde{m}=\exp \left(\tilde{n}^{\delta}\right) \\
\tilde{n} & \geq q^{m+1} \\
h^{m} / 4 & \geq \tilde{n}^{1-\eta} \\
(n-1)(h-1) m & \leq q / 4
\end{aligned}
$$




\begin{tabular}{|l|c||l|c|}
\hline \multicolumn{2}{|c||}{ Bipartite expander graph } & \multicolumn{2}{c|}{ PRG } \\
\hline \#left vertices & $q^{n}$ & Output size & $\tilde{m}$ \\
\hline \#right vertices & $q^{m+1}$ & PRG input & $\tilde{n}$ \\
\hline Set size & $\ell=h^{m}$ & $r$-wise indep. & $\tilde{n}^{1-\eta}$ \\
\hline Expansion & $A=d / 2+c$ & Robustness & $\ell \cdot c / d \geq \tilde{n}^{1-\eta}$ \\
\hline
\end{tabular}

Table 11. Parameters of the bipartite graph and the PRG.

Given the constant $0<\eta<1$, we can take a large enough constant integer $m>0$ such that

$$
m(1-\eta / 2) \geq(m+1)(1-\eta)+1
$$

Namely, we can take $m=\lceil 2(2-\eta) / \eta\rceil$. We take $q$ be the largest power of 2 smaller than $\tilde{n}^{1 /(m+1)}$, which gives:

$$
\tilde{n}^{1 /(m+1)} / 2<q \leq \tilde{n}^{1 /(m+1)}
$$

and Inequality (4) is satisfied. We take $h=\left\lceil q^{1-\eta / 2}\right\rceil$, which gives from (7) and (8):

$$
h^{m} \geq q^{m(1-\eta / 2)} \geq q \cdot q^{(m+1)(1-\eta)}>q \cdot\left(\tilde{n} / 2^{m+1}\right)^{1-\eta} \geq \tilde{n}^{1-\eta} \cdot q / 2^{m+1}
$$

For large enough $\tilde{n}$, we have $q / 2^{m+1} \geq 4$ (recall that $m$ is a constant), and therefore Inequality (5) is satisfied. We take the constant $\delta:=\eta /(4(m+1))$. We take $n=\left\lceil\tilde{n}^{\delta}\right\rceil$, so that Inequality (3) is satisfied (since $q>\exp (1)$ for large enough $\tilde{n}$ ). Finally, we have using (8):

$$
\begin{aligned}
(n-1)(h-1) m & \leq \tilde{n}^{\delta} \cdot q^{1-\eta / 2} \cdot m \leq\left(\tilde{n}^{1 /(m+1)}\right)^{\eta / 4} \cdot q^{1-\eta / 2} \cdot m \\
& \leq(2 q)^{\eta / 4} \cdot q^{1-\eta / 2} \cdot m \leq 2 m \cdot q^{1-\eta / 4} \leq\left(8 m / q^{\eta / 4}\right) \cdot q / 4
\end{aligned}
$$

For large enough $\tilde{n}$ we get $8 m / q^{\eta / 4} \leq 1$ and therefore Inequality (6) is satisfied. Finally, we have $d=q \leq \tilde{n}^{1 /(m+1)} \leq\left(\log ^{1 / \delta} \tilde{m}\right)^{1 /(m+1)} \leq \log ^{C} \tilde{m}$ for $C:=4 / \eta$.

\section{A.4 Concrete analysis of PRG based on [GUV09]}

The [GUV09] bipartite graph expander recalled in the previous section is defined by the following 5 parameters: $q, n, d, m$ and $h$. Therefore, finding the right parameters is not obvious. In the following we study two possible regimes.

Minimizing the input randomness. In this regime, we want to minimize the size of the input randomness of the $r$-wise independent PRG. We fix the parameter $m$. Therefore we must have $h^{m} \geq r$, so we take $h=\left\lceil r^{1 / m}\right\rceil$. The PRG takes as input $d q^{m} \leq q^{m+1}$ random bits and outputs $q^{n}$ pseudo-randoms, so we take $n=m+1$, so that the PRG is at least expanding. For minimal robustness, we take $A>d / 2$, which gives the condition $q>d>2(n-1)(h-1) m$. Therefore we take $d=q-1$ and $q$ the smallest prime greater than $2(n-1)(h-1) m+2$. Recall that the trivial construction based on polynomial evaluation can achieve $r$-wise independence and resistance against $r$ probes with $r(r+1)$ input randoms. To do better than the trivial construction, one must therefore ensure that $d q^{m}<r(r+1)$. As illustrated in Table 12, the PRG based on expander graph becomes better than the trivial construction only for $r \geq 2^{18}$, which requires at least $2^{36}$ input random bits. Therefore, if the goal is to use fewer TRNGs than the trivial construction, the construction of expander graphs based on [GUV09] is totally impractical.

Small $\boldsymbol{d}$ regime. In this regime, we want to minimize the degree $d$ for a given $r$, in order to minimize the time of pseudo-random generation. For simplicity we only require the $r$-wise independence. Therefore we can take $d=2(n-1) m(h-1)+1$ and we must have $h^{m} \geq r$; we take $n=m+1$. We provide the result in Table 13. For large values of $m$, we can use the approximation $h=r^{1 / m}$ and $d=2 m^{2} h$. We must therefore minimize the function $f(m)=2 m^{2} r^{1 / m}$, and we find that the function is minimized for $m=\log r$, which gives $d=\mathcal{O}\left(\log ^{2} r\right)$. Therefore expander graphs can be used to minimize the time generation of pseudo-randoms. 


\begin{tabular}{|l||c|c|c|c|}
\hline$r$-wise independence & $2^{18}$ & $2^{22}$ & $2^{30}$ & $2^{40}$ \\
\hline$m$ & 2 & 2 & 2 & 2 \\
\hline$h$ & 512 & 2048 & 32768 & $2^{20}$ \\
\hline$q$ & 4091 & 16381 & 262139 & $\simeq 2^{23}$ \\
\hline Number of input bits $d q^{m}$ & $2^{36}$ & $2^{42}$ & $2^{54}$ & $2^{69}$ \\
\hline
\end{tabular}

Table 12. Parameters of the PRG based on the [GUV09] bipartite expander graph.

\begin{tabular}{|c||c|ccc|c|c|c|c|c|c|c|c|c|c|c|c|c|c|}
\hline$d$ & 2 & 3 & $\cdots$ & 54 & 55 & 65 & 73 & 89 & 91 & 97 & 109 & 127 & 129 & 145 & 151 & 161 & 181 & 193 \\
\hline$r$ & 2 & 3 & $\cdots$ & 54 & 64 & 81 & 125 & 144 & 216 & 256 & 343 & 512 & 625 & 729 & 1024 & 1296 & 1331 & 2401 \\
\hline
\end{tabular}

Table 13. Maximal value of $r$-wise independence parameter $r$ achievable for a given degree $d$.

\section{B Definitions and Previous Work}

\section{B.1 Security Definition}

The PINI security notion. We actually use a slightly simplified definition compared to [CS18], in which the authors define $t$-PINI where $t_{1}+t_{2} \leq t$, where $t_{1}$ is the number of intermediate variables and $|\mathcal{O}|=t_{2}$, and we must have $|I| \leq t_{1}$. In our definition we do not set an upper bound on the total number of variables $t$ that must be simulated, as this appears to be unnecessary. Clearly a gadget that is PINI under our definition is $t$-PINI under [CS18] for any $t \in \mathbb{N}$. Conversely, a gadget that is $(n-1)$-PINI under [CS18] is actually $t$-PINI for any $t \in \mathbb{N}$, and therefore PINI under our definition. Namely it must be $t$-PINI for any $t<n$; and for $t \geq n$, if $t_{1}+t_{2} \geq n$, we can take $I=[1, n] \backslash \mathcal{O}$, and all intermediate variables can be perfectly simulated knowing all input shares from $I \cup \mathcal{O}=[1, n]$, with $|I|=n-|\mathcal{O}|=n-t_{2} \leq t_{1}$; therefore it is $t$-PINI also for any $t \geq n$.

Proof of Proposition 2 (PINI composition [CS18]). As in [CS18], we consider $l$ Gadgets $G_{1}, \cdots, G_{l}$ that we order as a direct acyclic graph from output to input in a reverse topological sort order. We assume that each gadget $G_{i}$ has $t_{i}$ internal probes and that the sum of all internal probes is equal to $t$. Furthermore, the last gadget $G_{1}$ has $\mathcal{O}$ output probes. We prove by recurrence on $i$ that the composition of PINI gadgets remains PINI.

If $i=1$, then there is only one gadget and this is straightforward since by assumption the gadget is PINI. Now we assume that the composition of gadgets $G_{1}, \cdots, G_{i}$ is PINI and we prove that the composition of gadgets $G_{1}, \cdots, G_{i+1}$ is still PINI. Since the composition of gadgets $G_{1}, \cdots, G_{i}$ is PINI, we get that for any set of $t_{1}+\cdots+t_{i}$ intermediate variables and any subset $\mathcal{O}$ of output indices, there exists a subset $I_{i} \subset[1, n]$ of input indices with $\left|I_{i}\right| \leq t_{1}+\cdots+t_{i}$ such that the $t_{1}+\cdots+t_{i}$ intermediate variables and the output shares $y_{\mid \mathcal{O}, \star}$ can be perfectly simulated from the input shares $x_{\mid I_{i} \cup \mathcal{O}, \star}$. Furthermore, since gadget $G_{i+1}$ is PINI, one can simulate Gadget $G_{i+1}$ with input shares corresponding to indices in $S_{i+1} \cup\left(I_{i} \cup \mathcal{O}\right)$ with $\left|S_{i+1}\right| \leq t_{i+1}$.

Therefore we can compose these simulators to perfectly simulate the composition of gadgets $G_{1}, \cdots, G_{i+1}$ from $I_{i+1} \cup \mathcal{O}$ with $I_{i+1}=S_{i+1} \cup I_{i}$, and since

$$
\left|I_{i+1}\right|=\left|S_{i+1} \cup I_{i}\right| \leq\left|S_{i+1}\right|+\left|I_{i}\right| \leq t_{i+1}+\left(t_{1}+\cdots+t_{i}\right) \leq t
$$

we conclude that the composition of $i+1$ gadgets PINI remains PINI.

For $i+1=l$, we get that we can perfectly simulate the composition of gadgets $G_{1}, \cdots, G_{l}$ from $I_{l} \cup \mathcal{O}$ with $\left|I_{l}\right|=\left|\bigcup_{i=1}^{i=l} S_{i}\right| \leq t_{1}+\cdots+t_{l} \leq t$. This terminates the proof.

\section{B.2 Basic gadgets SecMult and FullRefresh}

We recall in Algorithm 4 the SecMult gadget used in [RP10] for protecting AES against $t$-th order attacks. It is an extension to $\mathbb{F}_{2^{k}}$ of the original ISW countermeasure [ISW03] described in $\mathbb{F}_{2}$. The SecMult gadget was proven $t$-SNI in $\left[\mathrm{BBD}^{+} 16\right]$. 


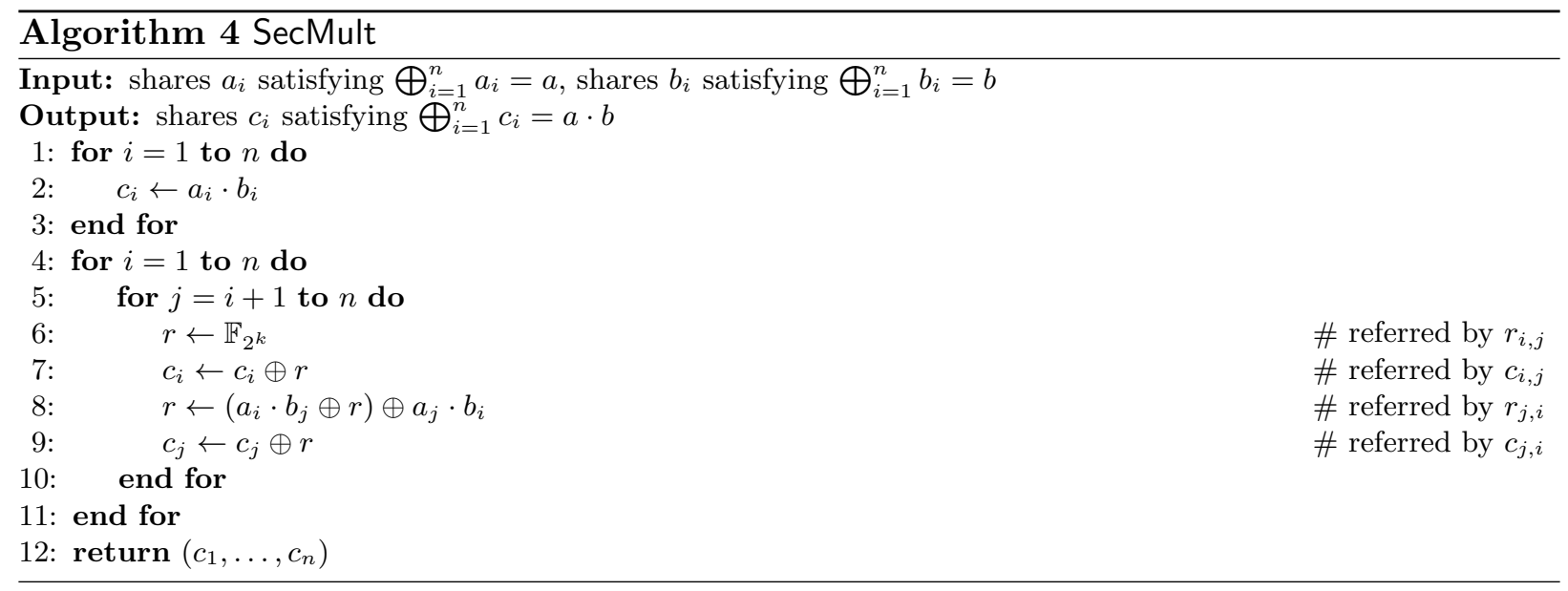

The mask refreshing gadget FullRefresh recalled in Algorithm 5 was introduced by Duc et. al in [DDF14]; it was proven $t$-SNI in $\left[\mathrm{BBD}^{+} 16\right]$.

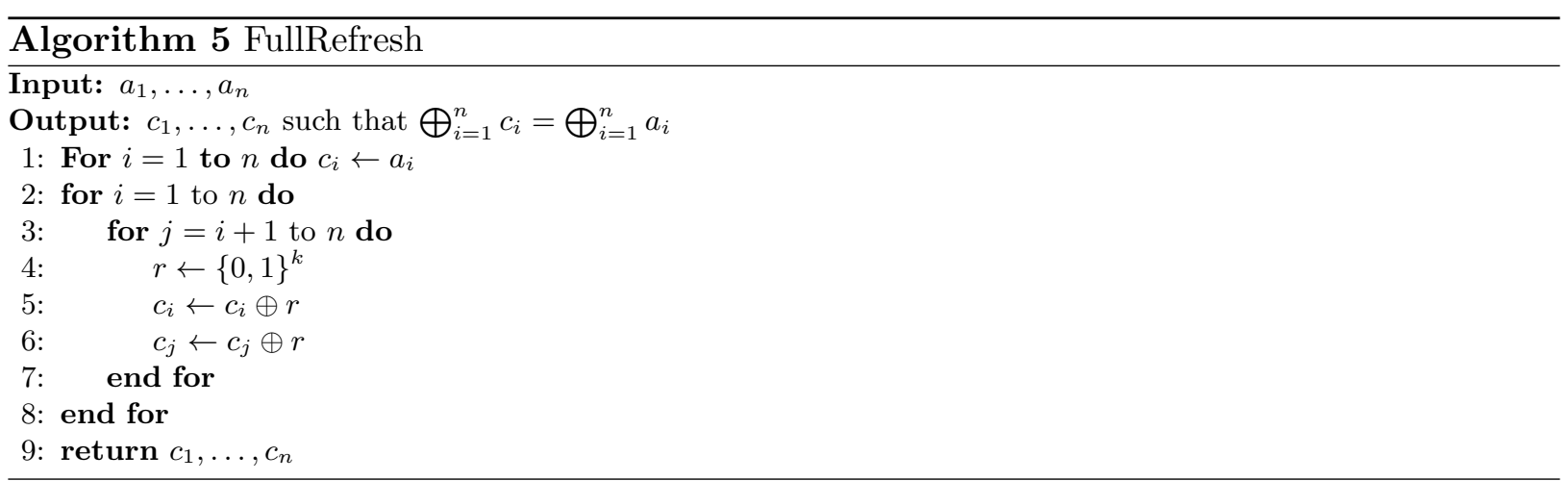

\section{B.3 Proof of Theorem 1 (Private implementation $\left[\mathrm{IKL}^{+} 13\right]$ )}

We start with the following simple Lemma. It shows that for a linear $r$-wise PRG, instead of randomly generating the $n$-bit seed and computing the PRG output on $r$ bits, one can first generate the $r$-bit output uniformly at random and then sample the $n$-bit seed, with the same joint distribution.

Lemma 19 (PRG input sampling). Let $G:\{0,1\}^{n} \rightarrow\{0,1\}^{m}$ be an $r$-wise independent linear $P R G$. Let $T \subset[m]$ be any subset of at most $r$ bits. Given any $\rho_{T} \in\{0,1\}^{|T|}$, one can efficiently sample $x^{\prime}$ such that $G_{T}\left(x^{\prime}\right)=\rho_{T}$, and moreover $\left(X, G_{T}(X)\right) \equiv\left(x^{\prime}, R_{T}\right)$ where $X \leftarrow\{0,1\}^{n}$ and $R \leftarrow\{0,1\}^{m}$.

Proof. Since $G$ is an $r$-wise independent PRG and $|T| \leq r$, the random variable $Y=G_{T}(X)$ is uniformly and independently distributed. This implies for any $\rho_{T} \in\{0,1\}^{|T|}$ :

$$
\operatorname{Pr}\left[G_{T}(X)=\rho_{T}\right]=2^{-|T|}=\operatorname{Pr}\left[X \in G_{T}^{-1}\left(\rho_{T}\right)\right]=\frac{\left|G_{T}^{-1}\left(\rho_{T}\right)\right|}{2^{n}}
$$

and therefore $\left|G_{T}^{-1}\left(\rho_{T}\right)\right|=2^{n-|T|}$ for all $\rho_{T} \in\{0,1\}^{|T|}$. Moreover we can efficiently sample $x^{\prime}$ uniformly at random in $G_{T}^{-1}\left(\rho_{T}\right)$ because the PRG is linear. When $\rho_{T} \leftarrow\{0,1\}^{|T|}$, we obtain that $x^{\prime}$ is uniformly distributed in $\{0,1\}^{n}$. This proves the lemma. 
Proof of Lemma 3 (Probing strong robust PRG). Let $T$ be the set corresponding to $S$ in definition 6 . From Lemma 19 , we have $\left(X, G_{T}(X)\right) \equiv\left(x^{\prime}, R_{T}\right)$ where $x^{\prime} \leftarrow G_{T}^{-1}\left(\rho_{T}\right)$; this gives:

$$
\left(G_{S}(X), G_{T}(X)\right) \equiv\left(G_{S}\left(x^{\prime}\right), R_{T}\right)
$$

From $|L \backslash T| \leq|L| \leq r-q|S|$ and the strong $(r, q, k)$-robustness of $G$, we have that $G_{L \backslash T}(X)$ is uniform and independent from $\left(G_{S}(X), G_{T}(X)\right)$. Therefore:

$$
\left[\left(G_{L \backslash T}(X), G_{T}(X)\right) \mid G_{S}(X)=g_{S}, G_{T}(X)=g_{T}\right] \equiv\left[R_{L \cup T} \mid R_{T}=g_{T}\right]
$$

Since the distribution of $x^{\prime}$ only depends on $g_{T}$, we have:

$$
\left[R_{L \cup T} \mid R_{T}=g_{T}\right] \equiv\left[R_{L \cup T} \mid G_{S}\left(x^{\prime}\right)=g_{S}, R_{T}=g_{T}\right]
$$

Combining the two equations, we get:

$$
\left[G_{L \cup T}(X) \mid G_{S}(X)=g_{S}, G_{T}(X)=g_{T}\right] \equiv\left[R_{L \cup T} \mid G_{S}\left(x^{\prime}\right)=g_{S}, R_{T}=g_{T}\right]
$$

Combining with the first equation, we get

$$
\left(G_{L \cup T}(X), G_{S}(X), G_{T}(X)\right) \equiv\left(R_{L \cup T}, G_{S}\left(x^{\prime}\right), R_{T}\right)
$$

and therefore:

$$
\left(G_{L \cup T}(X), G_{S}(X)\right) \equiv\left(R_{L \cup T}, G_{S}\left(x^{\prime}\right)\right)
$$

Therefore the distribution of $Y=G_{L \cup T}(X)$ is uniform in $\{0,1\}^{|L \cup T|}$ when $X \leftarrow\{0,1\}^{n}$ and moreover $G_{S}(X)$ can be efficiently simulated given $Y_{T}$ only.

Proof of Theorem 1. Consider the $k$ probes in the circuit $C^{\prime}$ distributed as follows: let $t$ be the number of probes in the PRG and $k-t$ be the number of probes in the circuit $C$. Since $C$ makes an $\ell$-local use of its randomness, the probes in $C$ can depend on at most $(k-t) \cdot \ell$ bits of randomness. From Lemma 3 , the output of $r-q t$ bits of the PRG can be replaced by a TRNG, while at most $q t$ bits of output are required for the simulation of the $t$ probes of the PRG. Therefore, we must ensure:

$$
(k-t) \cdot \ell \leq r-q t
$$

If $q \leq \ell$, then from $r \geq k \ell$ we get $(k-t) \cdot \ell=k \ell-\ell t \leq r-q t$ as required. If $q \geq \ell$ then from $r \geq k q$ we get $(k-t) \cdot \ell \leq(k-t) \cdot q=k q-q t \leq r-q t$ as required. Eventually, the $k$ probes in $C^{\prime}$ can be perfectly simulated by using

$$
q \cdot t+(k-t) \leq q \cdot t+q(k-t) \leq q k
$$

probes in the original $q k$-private circuit $C$. Therefore, the circuit $C^{\prime}$ is a $k$-private implementation of $f$.

\section{B.4 Proof of Lemma 5 (Private circuit with PRG)}

We first compute the concrete locality $\ell$ of the SecMultFLR algorithm. We assume that $n \geq 3$. The SecMult gadget takes as input two sets of shares $a_{i}$ and $b_{i}$ for $1 \leq i \leq n$ and we assume that those shares correspond to previous outputs of a locality refresh LR. Therefore those shares are such that for $1 \leq i \leq n-1$, the shares $a_{i}$ and $b_{i}$ are random values and the shares $a_{n}$ and $b_{n}$ depend respectively on the $n-1$ random values $a_{i}$ and $b_{i}$. As a consequence, the variable $a_{n} b_{n}$ which is computed within the SecMult Gadget depends on $\ell_{I}=2(n-1)$ random values, i.e. on all input randoms.

Furthermore in the SecMult Gadget, $n(n-1) / 2$ fresh randoms are generated. The variables which have the largest dependence to the fresh randoms are the output shares $c_{i}$; one can see that 
each output $c_{i}$ depends on exactly $n-1$ fresh randoms. In particular, the output $c_{n}$ which includes the value $a_{n} b_{n}$ depends on $n-1$ fresh randoms and on the $\ell_{I}$ input randoms.

Now we investigate the locality refreshing LR performed after SecMult. Within LR, all outputs $y_{i}$ are fresh randoms i.e. they depend on one random value $s_{i}$, except for the last output $y_{n}$ which depends on those fresh randoms $s_{i}$ and on all inputs $c_{i}$. More precisely, we have: $y_{n}^{(0)}=c_{n}$ and $y_{n}^{(i)}=y_{n}^{(i-1)} \oplus\left(c_{i} \oplus s_{i}\right)$, where the last output is $y_{n}=y_{n}^{(n-1)}$. Therefore we must investigate all intermediate values $y_{n}^{(i)}$ for $0 \leq i \leq n-1$ to determine which one depends on the most random values. Note that since all values $y_{n}^{(i)}$ include the share $c_{n}$, they all depend on at least $\ell_{I}+(n-1)$ random values.

By construction of the $c_{i}$ output shares in the SecMult Gadget, each $c_{i}$ depends on exactly $n-1$ fresh random values. Furthermore, if we take an arbitrary share $c_{i}$ with $i<n$, we know that $c_{i}$ and $c_{n}$ have exactly one random value in common. Therefore knowing that the shares $c_{n}$ and $c_{i}$ depend respectively on $n-1$ random values, the value $c_{n} \oplus c_{i}$ will depend on $(n-1)+(n-1)-2=2 n-4$ random values since the $\oplus$ operation removes the common random in both $c_{n}$ and $c_{i}$. Thus, for example, the value $y_{n}^{(1)}=c_{n} \oplus\left(c_{1} \oplus s_{1}\right)$ depends on $\ell_{I}+2 n-4+1$ random values since a new random $s_{1}$ is included. With $\ell_{I}=2(n-1)$, we get that $y_{n}^{(1)}$ depends on $4 n-5$ random values.

More generally, if we consider the xor of $i+1$ output shares $c_{k}$, we know that they have exactly $\sum_{j=1}^{i} j$ random values in common among the fresh random values. Therefore, if we omit for now the dependence to input randoms $\ell_{I}$, the variable $c_{n} \oplus c_{1} \oplus \cdots \oplus c_{i}$ depends on

$$
\ell_{C}=(i+1)(n-1)-2 \sum_{j=1}^{i} j=\sum_{j=0}^{i}(n-1-2 j)
$$

random values. The value $\ell_{C}$ increases with the number of shares $i$ provided that $2 i \leq n-1$ and is maximized for $i=\lfloor(n-1) / 2\rfloor$, where we have:

$$
\ell_{C}=\sum_{j=0}^{\lfloor(n-1) / 2\rfloor}(n-1-2 j)=\left\lfloor\frac{n^{2}}{4}\right\rfloor .
$$

Therefore the maximum dependence with random values is reached with the variable $c_{n} \oplus c_{1} \oplus$ $\cdots \oplus c_{\lfloor(n-1) / 2\rfloor}$. Therefore in the locality refreshing LR, we deduce that the intermediate variable which has the largest dependency to random values is

$$
y_{n}^{(\lfloor(n-1) / 2\rfloor)}=c_{n} \oplus\left(s_{1} \oplus c_{1}\right) \oplus \cdots \oplus\left(s_{(\lfloor(n-1) / 2\rfloor)} \oplus c_{(\lfloor(n-1) / 2\rfloor)}\right) .
$$

As a consequence, this value has additional dependence to $\ell_{R}$ random values $s_{i}$ where $\ell_{R}=\left\lfloor\frac{n-1}{2}\right\rfloor$. In conclusion, the locality parameter $\ell$ is as follows:

$$
\ell=\ell_{I}+\ell_{C}+\ell_{R}=2(n-1)+\left\lfloor\frac{n^{2}}{4}\right\rfloor+\left\lfloor\frac{n-1}{2}\right\rfloor .
$$

The SecMult algorithm followed by a final locality refresh (SecMultFLR) is therefore an $\ell$-local gadget with $\ell=n^{2} / 4+5 n / 2-c$, where $c=3$ for even $n$, and $c=11 / 4$ for odd $n$. With $n=t+1$ we have $\ell=\mathcal{O}\left(t^{2}\right)$

The trivial construction of xoring $(t+1) r$-wise independent PRGs based on polynomial evaluation, use $(t+1) \cdot r \cdot \log _{2}|\mathbb{F}|$ bits of randomness and can generate $m$ bits of output, where $m \leq|\mathbb{F}|$. From Theorem 1 we must set $r=t \cdot \ell$; with $\ell=\mathcal{O}\left(t^{2}\right)$, we get $r=\mathcal{O}\left(t^{3}\right)$. Let $s$ be the original unprotected circuit size. We need $m=\mathcal{O}\left(s \cdot t^{2}\right)$ bits of randomness with the ISW construction, therefore we can have $\log _{2}|\mathbb{F}|=\mathcal{O}(\log s+\log t)$, and therefore the total amount of input randomness with the robust PRG is $\mathcal{O}\left(t^{4} \cdot(\log s+\log t)\right)=\tilde{\mathcal{O}}\left(t^{4}\right)$. Each pseudo-random generation also takes $\tilde{\mathcal{O}}\left(t^{4}\right)$ time; therefore the circuit runs in time $\tilde{\mathcal{O}}\left(s \cdot t^{6}\right)$ with the trivial construction. 


\section{$\mathrm{C}$ Improving the locality of the multiplication gadget}

\section{C.1 Proof of Lemma 8 (locality of SecMultILR)}

We consider the SecMultILR algorithm which takes as input two sets of shares $a_{i}$ and $b_{i}$ for $1 \leq i \leq n$ and we assume that those shares are already locality refreshed. Therefore the shares $a_{i}$ and $b_{i}$ are random values for $1 \leq i \leq n-1$ and the shares $a_{n}$ and $b_{n}$ depend respectively on the $n-1$ random values $a_{i}$ and $b_{i}$. As a consequence, the variable $a_{n} b_{n}$ which is computed within the gadget depends on $2(n-1)$ random values.

The SecMultILR algorithm consists of a main loop divided into two sub-loops: a first sub-loop $A$ performing the ISW multiplication (lines 5 to 10), and a second loop $B$ performing the locality refreshing (lines 11 to 15 ).

In the following, we assume that $n \geq 3$ and we prove by recurrence for $2 \leq j \leq n-1$ that the variable $c_{j, j-2}$ (or $c_{2,1}$ if $j=2$ ) in loop $B$ has a dependence to $4 j-3$ random values, which is the maximal dependence to random values at loop $j$.

Base case: for $j=2$, in Loop $A$, the variable $c_{2,1}=a_{2} b_{2} \oplus\left(a_{1} b_{2} \oplus r_{1,2} \oplus a_{2} b_{1}\right)$ depends on the 4 input random values $a_{1}, a_{2}, b_{1}, b_{2}$ and on the fresh random $r_{1,2}$ with a total of 5 random values. Furthermore in Loop $B$, the variable $c_{2,1}=\left(a_{2} b_{2} \oplus a_{1} b_{2} \oplus r_{1,2} \oplus a_{2} b_{1}\right) \oplus\left(\left(a_{1} b_{1} \oplus r_{1,2}\right) \oplus s_{1,2}\right)$ still depends on the 4 input random values $a_{1}, a_{2}, b_{1}, b_{2}$, and on the fresh random value $s_{1,2}$, but it does not depend anymore on $r_{1,2}$ which disappears with the $\oplus$ operation. Therefore, the variable $c_{2,1}$ in loop $A$ and $B$ has a dependence to $5=4 j-3$ random values with $j=2$.

Recurrence step: now, we assume that for $j=k$, the value $c_{k, k-2}$ in Loop $A$ and $B$ has a maximal dependence to $4 k-3$ random values and we show that for $j=k+1$, the value $c_{k+1, k-1}$ in Loop $B$ has a dependence to $4(k+1)-3=4 k+1$ random values. We start by analyzing Loop $A$ at round $j=k+1$, and more precisely we consider the values $c_{k, k+1}$ and $c_{k+1, k}$ i.e. values at lines 7 and 9 at loop $i=j-1=k$. The value $c_{k+1, k}$ depends on all inputs $a_{i}$ and $b_{i}$ for $1 \leq i \leq k+1$ and on $k$ fresh random values $r_{1, k+1}, \ldots, r_{k, k+1}$, which gives a dependence to $2(k+1)+k=3 k+2$ random values, which is smaller than $4 k+1$. Furthermore, the value $c_{k, k+1}$ is such that $c_{k, k+1}=c_{k, k-1} \oplus r_{k, k+1}$ and since by recurrence hypothesis the value $c_{k, k-1}$ has a dependence to at most $4 k-3$ random values, the value $c_{k, k+1}$ depends on $(4 k-3)+1=4 k-2$ random values, which is still smaller than $4 k+1$. Therefore in Loop $A$, we have shown that the maximal dependence to random values is smaller than $4 k+1$, with a dependence to $3 k+2$ random values for the variable $c_{k+1, k}$ at round $j=k+1$.

Now we consider Loop $B$. At line 13 of the SecMultILR algorithm, a xor between $c_{k+1, k}$ and all $c_{i, k+1}$ computed in Loop $A$ is performed for $1 \leq i \leq k$, to which $j-1=k$ fresh random values $s_{1, k+1}, \ldots, s_{k, k+1}$ are added. By construction, for $1 \leq i \leq k-1$ we have $c_{i, k+1}=s_{i, k} \oplus r_{i, k+1}$, which means that the computation at line 13 is $c_{k+1, i} \oplus\left(c_{i, k+1} \oplus s_{i, k+1}\right)=c_{k+1, i} \oplus\left(s_{i, k} \oplus r_{i, k+1} \oplus s_{i, k+1}\right)$. Therefore, at each loop $i$, the dependence to random $r_{i, k+1}$ is removed (since the random $r_{i, k+1}$ also belongs to $c_{k+1, i}$ ), but two new dependencies are created with the adding of random values $s_{i, k}$ and $s_{i, k+1}$; which overall amounts to adding a dependence to one random value at each loop $i$. Note that this is true for $i \leq k-1$ since at round $i=k$ the dependence to all randoms $s_{i, k}$ is removed (since they all belong to $c_{k, k+1}$ ). Therefore, for $j=k+1$ the number of random values to which $c_{k+1, i}$ depends goes increasing by one until $i=k-1$. As a consequence, the value $c_{k+1, k-1}$ depends on $k-1$ new randoms, to which we add the original dependence of the variable $c_{k+1, k}$ at end of loop $A$ (the first $c_{j}$ value at loop $B$ ) which is, as previously shown, of $3 k+2$ random values. We therefore get that the variable $c_{k+1, k-1}$ depends on $(k-1)+(3 k+2)=4 k+1$ random values and this asserts the recursive hypothesis.

As a consequence, one has shown that in loops $A$ and $B$, the variable $c_{j, j-2}$ has a dependence to $4 j-3$ random values for $2 \leq j \leq n-1$. In particular, for $j=n-1$, the value $c_{n-1, n-3}$ has a dependence to $4(n-1)-3=4 n-7$ random values.

Last step: for the specific case $j=n$, one has to consider the fact that the input variables $a_{n}$ and $b_{n}$ are not random values but depend each on the $n-1$ random values $a_{i}$ and $b_{i}$. Therefore, when counting the randomness of $c_{n, n-2}$, one has to remove both values $a_{n}$ and $b_{n}$ which are 
already counted, that is, the variable $c_{n, n-2}$ has a dependence to $4 n-3-2=4 n-5$ random values, and this variable has the largest dependence to random values in the SecMultILR algorithm. As a conclusion, we have shown that the locality parameter is $\ell=4 n-5$ for $n \geq 3$.

\section{C.2 Proof of Theorem 3 (Completeness of SecMultILR)}

The SecMultILR algorithm consists of a main loop which performs two loops: a first loop $A$ from line 5 to 10 which is the same as in the original SecMult and a second loop $B$ from lines 11 to 15 which corresponds to a locality refresh of outputs from loop $A$. We proceed by recurrence on $n$. For $n=1$, the algorithm outputs $c_{1}=a_{1} \cdot b_{1}$ which is correct. Now we assume that the relation is true for $j=k$ and we show that it is still correct for $j=k+1$.

The first loop $A$ for $j=k+1$ will compute the following:

$$
c_{k+1}=a_{k+1} b_{k+1} \oplus \bigoplus_{i=1}^{k}\left(a_{i} b_{k+1} \oplus a_{k+1} b_{i} \oplus r_{i}\right) \quad \text { and } \quad c_{i}=c_{i}^{\prime} \oplus r_{i} \text { for } 1 \leq i \leq k
$$

where the $c_{i}^{\prime}$ correspond to outputs after round $j=k$. Therefore, the sum between all new $c_{i}$ and $c_{k+1}$ gives:

$$
\bigoplus_{i=1}^{k+1} c_{i}=a_{k+1} b_{k+1} \oplus \bigoplus_{i=1}^{k}\left(a_{i} b_{k+1} \oplus a_{k+1} b_{i}\right) \oplus \bigoplus_{i=1}^{k} c_{i}^{\prime}
$$

Since by recurrence assumption we have $\bigoplus_{i=1}^{k} c_{i}^{\prime}=\bigoplus_{i=1, j=1}^{k} a_{i} b_{j}$, we deduce that

$$
\begin{aligned}
\bigoplus_{i=1}^{k+1} c_{i} & =a_{k+1} b_{k+1} \oplus \bigoplus_{i=1}^{k}\left(a_{i} b_{k+1} \oplus a_{k+1} b_{i}\right) \oplus \bigoplus_{i=1, j=1}^{k} a_{i} b_{j} \\
& =\bigoplus_{i=1, j=1}^{k+1} a_{i} b_{j}=\left(\bigoplus_{i=1}^{k+1} a_{i}\right) \cdot\left(\bigoplus_{i=1}^{k+1} b_{i}\right)
\end{aligned}
$$

Thus, at the end of Loop $A$ at round $j=k+1$, the completeness of SecMultILR is verified. After Loop $A$ has been performed, the second loop $B$ is nothing but a refreshing of the outputs of the first loop $A$. Therefore the outputs of Loop $B$ verify the same property as the outputs of Loop $A$, which terminates the proof.

\section{C.3 Proof of Theorem 4 ( $t$-SNI of SecMultILR)}

The proof is based on the $t$-SNI property of the original SecMult algorithm (see Appendix B.2). We describe a sequence of games.

Game 0: we generate the random variables as in the original circuit. Using all inputs $a_{i}$ and $b_{j}$, we can simulate all intermediate variables.

Game 1: we modify the distribution of the variables $s_{i j}$ as follows. Instead of letting $s_{i j} \leftarrow \mathbb{F}_{2^{k}}$, we let $s_{i j} \leftarrow s_{i j}^{\prime} \oplus c_{i j}$, where $c_{i j}$ is the value of $c_{i}$ at Line 13 , and $s_{i j}^{\prime} \leftarrow \mathbb{F}_{2^{k}}$. Since we have replaced a subset of random variables by identically distributed random variables, the adversary's view has the same distribution as in Game 0. Moreover, in Game 1, the following operations are now performed:

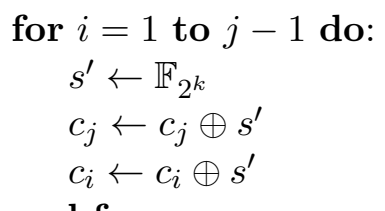

end for 
We see that the operations correspond to the mask refreshing from [RP10], applied to the first $i$ shares $c_{1}, \ldots, c_{j}$.

Game 2: we modify the simulation of the intermediate variables as follows. We observe that the circuit is linear in the variables $s_{i j}^{\prime}$; more precisely, any intermediate variable can be written as $f\left(\left(a_{i}\right)_{i},\left(b_{i}\right)_{i},\left(r_{i j}\right)_{i j}\right) \oplus g\left(\left(s_{i j}^{\prime}\right)_{i j}\right)$ for some function $f$ and some linear function $g$. Note that the function $f$ corresponds to the value of the intermediate variable with all $s_{i j}^{\prime}$ fixed to 0 . To simulate such intermediate variable, we therefore generate the randomness as in Game 1, except that all $s_{i j}^{\prime}$ are first set to 0; we then generate the randoms $s_{i j}^{\prime}$ as in the original circuit, and compute the function $g$ to simulate the corresponding intermediate variable. Since the distribution of the intermediate variable remains the same, the adversary's view has the same distribution as in Game 1.

Game 3: finally, we modify the way the function $f$ is computed for a given intermediate variable. The function $f$ actually corresponds to the original SecMult algorithm without the internal locality refreshing. Since the SecMult gadget is $t$-SNI, the $t_{1}$ intermediate variables and the output variables $c_{\mid O}$ can be perfectly simulated from the knowledge of $a_{\mid I}$ and $b_{\mid J}$, with $|I| \leq t_{1}$ and $|J| \leq t_{1}$, under the condition $t_{1}+|O| \leq t$. Therefore, the adversary's view for the full circuit can be perfectly simulated from the same $a_{\mid I}$ and $b_{\mid J}$.

\section{C.4 The SecMultILR2 algorithm}

We provide below in Algorithm 6 the SecMultILR2 algorithm.

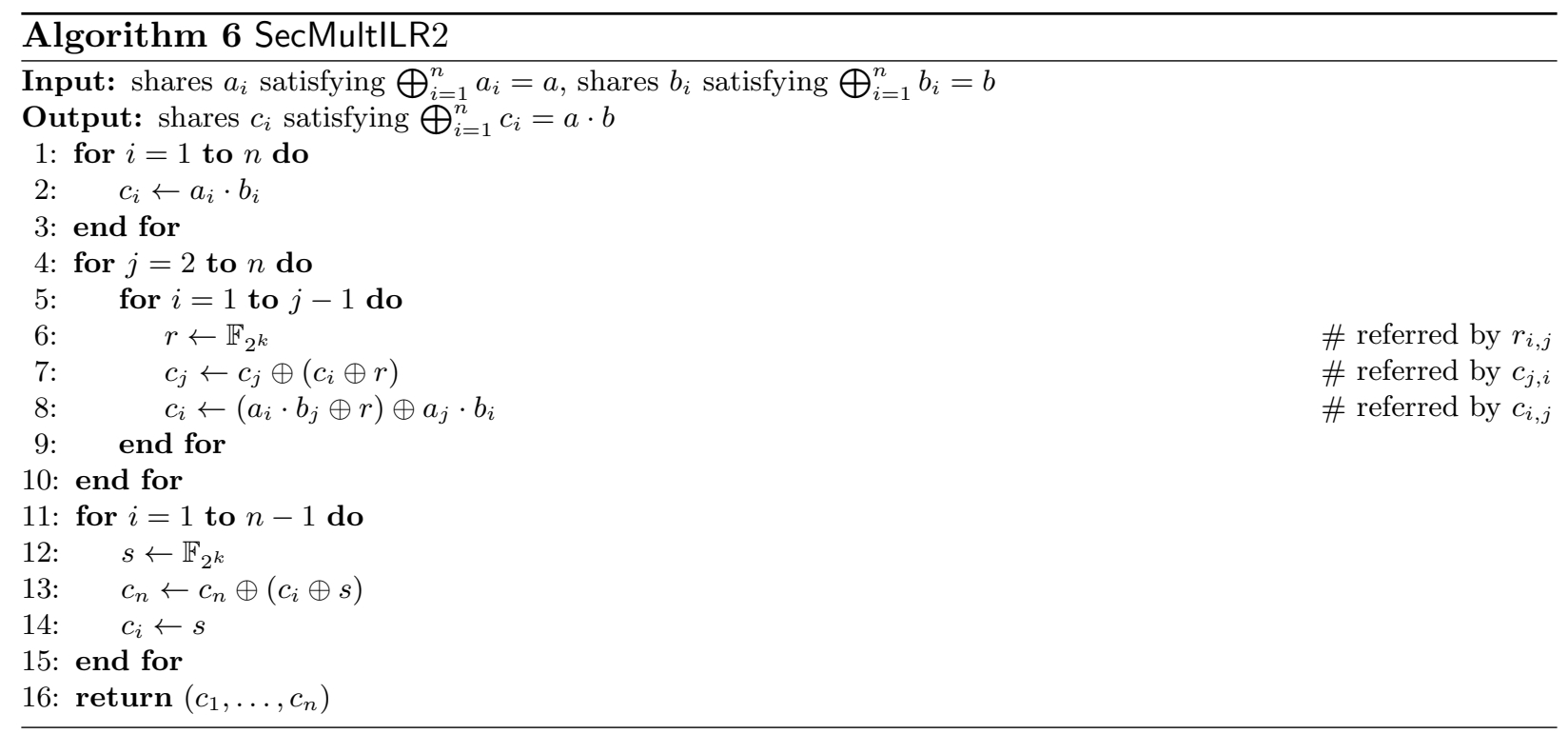

\section{C.5 Proof of Lemma 10 (Locality of SecMultILR2)}

We consider the SecMultILR2 algorithm which takes as input two sets of shares $a_{i}$ and $b_{i}$ for $1 \leq i \leq n$ and we assume that those shares correspond to previous outputs of a locality refreshing LR. Therefore the shares $a_{i}$ and $b_{i}$ are random values for $1 \leq i \leq n-1$ and the shares $a_{n}$ and $b_{n}$ depend respectively on the $n-1$ random values $a_{i}$ and $b_{i}$. As a consequence, the variable $a_{n} b_{n}$ which is computed within the gadget depends on $2(n-1)$ random values.

The SecMultILR2 algorithm consists of a main loop $A$ performing a slightly modified ISW multiplication (lines 4 to 10), and a final loop $B$ performing the refresh mask $R$ (lines 11 to 15 ). As we will see, for $2 \leq j \leq n$ the variable which depends on the most random numbers is the variable $c_{j}$ in Loop $A$ (line 7 ) for $i=j-2$, i.e. $c_{j, j-2}$. In the following, we assume that $n \geq 3$ and we prove 
that the variable $c_{j, j-2}$ in loop $A$ has a dependence to $4 j-4$ random values, which is the maximal dependence to random values at loop $j$.

At line 7 , the $c_{j}$ variable is updated at each round $i$, for $1 \leq i \leq j-1$. More precisely, we have that the last value $c_{j}$ is equal to

$$
c_{j, j-1}=c_{j, j-2} \oplus\left(c_{j-1, j-2} \oplus r_{j-1, j}\right) \quad \text { where } \quad c_{j, j-2}=a_{j} b_{j} \oplus \bigoplus_{i=1}^{j-2}\left(c_{i, j-1} \oplus r_{i, j}\right) .
$$

We start by analyzing the variable $c_{j, j-2}$. By construction, the variable $c_{j, j-2}$ depends on all input random variables $a_{i}$ and $b_{i}$ for $1 \leq i \leq j$, since it depends on $a_{j} b_{j}$ and on all intermediate values $c_{i, j-1}$ which themselves depend on $a_{j-1}, b_{j-1}, a_{i}$ and $b_{i}$ for $1 \leq i \leq j-2$. Therefore the variable $c_{j, j-2}$ depends on $2 j$ random input values. Now we investigate the dependence of $c_{j, j-2}$ to fresh random values $r$. All variables $c_{i, j-1}$ have a simple structure, i.e. $c_{i, j-1}=\left(a_{i} b_{j-1} \oplus\right.$ $\left.r_{i, j-1}\right) \oplus a_{j-1} b_{i}$ and they depend on one fresh random only, which is $r_{i, j-1}$. Therefore, the variable $c_{i, j-1} \oplus r_{i, j}$ depends on two fresh random values $r_{i, j-1}$ and $r_{i, j}$, and one deduces that the xor $\bigoplus_{i=1}^{j-2}\left(c_{i, j-1} \oplus r_{i, j}\right)$ depends on $2(j-2)$ fresh randoms. Thus in total, the variable $c_{j, j-2}$ depends on $2(j-2)+2 j=4 j-4$ random variables.

Now, we analyze the variable $c_{j, j-1}$ which is equal to $c_{j, j-2} \oplus\left(c_{j-1, j-2} \oplus r_{j-1, j}\right)$. By construction, the variable $c_{j-1, j-2}$ contains the xor of all intermediate variables $c_{i, j-1}$ for $1 \leq i \leq j-2$ (thus contains the xor of the $j-2$ randoms $r_{i, j-1}$ ), which are themselves contained in $c_{j, j-2}$. Therefore, the xor between $\left(c_{j-1, j-2} \oplus r_{j-1, j}\right)$ and $c_{j, j-2}$ removes the dependence to $j-2$ fresh random values $r_{1, j-1}, \ldots, r_{j-2, j-1}$ and adds only one dependence to a fresh random $r_{j-1, j}$. As a consequence, the variable $c_{j, j-1}$ has a dependence to $4 j-4-(j-2)+1=3 j-1$ random values, and we have $3 j-1 \geq 4 j-4$ for $j \geq 3$.

Next, we observe that the final loop $B$ performing the refresh mask $R$ does not increase the locality since at each loop on $i$, the operation $c_{n} \leftarrow c_{n} \oplus\left(c_{i} \oplus r\right)$ adds one fresh random $r$ but also removes one fresh random $r_{i, n}$ which appears in $c_{i}$ and $c_{n}$.

As a consequence, one has shown that the maximal dependence to random values is reached in loop $A$ with the variable $c_{j, j-2}$ which has a dependence to $4 j-4$ random values for $2 \leq j \leq n-1$. In particular, for $j=n-1$, the value $c_{n-1, n-3}$ has a dependence to $4(n-1)-4=4 n-8$ random values.

For the specific case $j=n$, one has to consider the fact that the input variables $a_{n}$ and $b_{n}$ are not random values but depend each on the $n-1$ random values $a_{i}$ and $b_{i}$. Therefore, when counting the randomness of $c_{n, n-2}$, one has to remove both values $a_{n}$ and $b_{n}$ which are already counted, that is, the variable $c_{n, n-2}$ has a dependence to $4 n-4-2=4 n-6$ random values, and this variable has the largest dependence to random values in the SecMultILR2 algorithm.

As a conclusion, we have shown that the locality parameter is $\ell=4 n-6$ for $n \geq 3$ and since $n=t+1$ we have that $\ell=\mathcal{O}(t)$ which concludes the proof.

\section{C.6 Proof of Theorem 5 (Completeness of SecMultILR2)}

The SecMultILR2 algorithm consists of a main loop $A$ performing a slightly modified ISW multiplication (lines 4 to 10), and a final loop $B$ performing the refresh mask $R$ (lines 11 to 15 ).

We proceed by recurrence on $n$. For $n=1$, the algorithm outputs $c_{1}=a_{1} \cdot b_{1}$ which is correct. Now we assume that the relation is true for $j=k$ and we show that it is still correct for $j=k+1$.

The first loop $A$ for $j=k+1$ will compute the following:

$$
c_{k+1}=a_{k+1} b_{k+1} \oplus \bigoplus_{i=1}^{k} r_{i} \oplus \bigoplus_{i=1}^{k} c_{i}
$$

Since by recurrence assumption we have $\bigoplus_{i=1}^{k} c_{i}=\bigoplus_{i=1, j=1}^{k} a_{i} b_{j}$ we deduce that $c_{k+1}=a_{k+1} b_{k+1} \oplus$ $\bigoplus_{i=1}^{k} r_{i} \oplus \bigoplus_{i=1, j=1}^{k} a_{i} b_{j}$. Furthermore, each new $c_{i}$ for $1 \leq i \leq k$ is such that: $c_{i}=a_{i} b_{k+1} \oplus a_{k+1} b_{i} \oplus$ 


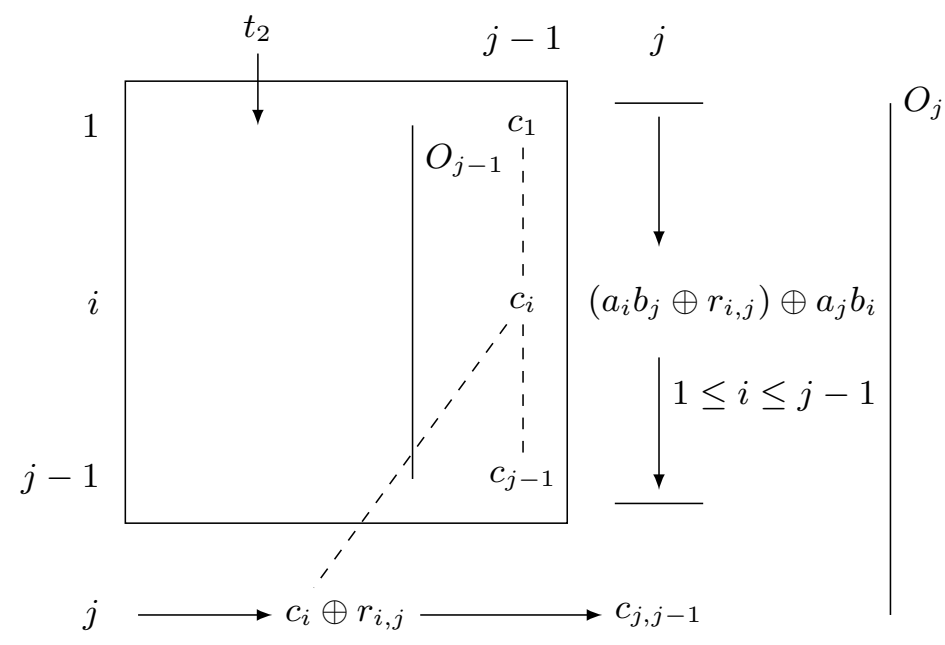

Fig. 10. Illustration of the $t$-NI proof. We maintain a list $L$ of the variables in $\left\{r_{i, j}, c_{i} \oplus r_{i, j}, a_{i} \cdot b_{j} \oplus r_{i, j},\left(a_{i} \cdot b_{j} \oplus\right.\right.$ $\left.\left.r_{i, j}\right) \oplus a_{j} \cdot b_{i}\right\}$ for $1 \leq i \leq j-1$ that must be simulated. This includes all variables that are directly probed, and all output variables $\left(a_{i} \cdot b_{j} \oplus r_{i, j}\right) \oplus a_{j} \cdot b_{i}$ such that $i \in O_{j}$. Moreover, if $j \in O_{j}$ or any variable $c_{j, i}$ is probed, we include in $L$ all variables $c_{i} \oplus r_{i, j}$, so that we can simulate all intermediate variables $c_{j, i}$.

$r_{i}$. Therefore, the sum between all new $c_{i}$ and $c_{k+1}$ gives:

$$
\begin{aligned}
\bigoplus_{i=1}^{k+1} c_{i} & =a_{k+1} b_{k+1} \oplus \bigoplus_{i=1, j=1}^{k} a_{i} b_{j} \oplus \bigoplus_{i=1}^{k}\left(a_{i} b_{k+1} \oplus a_{k+1} b_{i}\right) \\
& =\bigoplus_{i=1, j=1}^{k+1} a_{i} b_{j}=\left(\bigoplus_{i=1}^{k+1} a_{i}\right) \cdot\left(\bigoplus_{i=1}^{k+1} b_{i}\right)
\end{aligned}
$$

Thus, at the end of Loop $A$, the completeness of SecMultILR2 is verified and therefore the property is true for all $k \leq n$, in particular for $k=n$. After Loop $A$ has been performed, the second loop $B$ is nothing but a refreshing of the outputs of the first loop $A$. Therefore the outputs of Loop $B$ verify the same property as the outputs of Loop $A$, which terminates the proof.

\section{C.7 Proof of Theorem 6 ( $t$-SNI of SecMultILR2)}

The proof of Theorem 6 is relatively complex. We prove two intermediate lemmas below that consider some part of the SecMultILR2 algorithm.

Lemma 20 (t-NI property). Consider Algorithm 6 without lines 11 to 15. We consider an adversary who does not probe the variables $a_{i} b_{j}$ for $i \neq j$. Any set of $t_{n}$ intermediate variables and $c_{\mid O}$ for any $O \subset[1, n]$, can be perfectly simulated from $a_{\mid X \cup O}$ and $b_{\mid X \cup O}$, for some subset $X$ with $|X| \leq t_{n}$

Proof. We prove Lemma 20 by recurrence on the number of input shares $n$. See Figure 10 for an illustration of the proof. As written in Lemma 20, we assume that intermediate variables $a_{i} b_{j}$ for any $i \neq j$ are not probed.

Base Case: The call to Algorithm 6 (without lines 11 to 15) with $n=2$ amounts to performing the following computations: $c_{2,1}=a_{2} b_{2} \oplus\left(a_{1} b_{1} \oplus r_{1,2}\right)$ and $c_{1,2}=\left(a_{1} b_{2} \oplus r_{1,2}\right) \oplus a_{2} b_{1}$. If $t_{n}+|O| \geq 2$, then we can perfectly simulate all variables from the knowledge of the inputs $a_{1}, a_{2}, b_{1}$ and $b_{2}$. If we have $t_{n}+|O|<2$, three cases arise:

- If $t_{n}=0$ and $|O|=0$, then no variable is probed and nothing has to be simulated. Thus, the set $X$ is left empty and $|X|=0 \leq t_{n}$. 
- If $t_{n}=1$, then $|O|=0$ and no output variable is probed. In this case, if the probed variable is $a_{1}, b_{1}$ or $a_{1} b_{1}$ (resp. $a_{2}, b_{2}$ or $a_{2} b_{2}$ ), we put in $X$ the index 1 (resp. the index 2) and the latter can trivially be perfectly simulated from $a_{\mid X}$ and $b_{\mid X}$. Thus, in this case we have $|X|=1 \leq t_{n}$. If the probed variable is $r_{1,2}$ or $a_{1} b_{1} \oplus r_{1,2}$ or $a_{1} b_{2} \oplus r_{1,2}$, then the random $r_{1,2}$ can play the role of a one-time pad and the probed variable has a uniform distribution and can be simulated by generating a random. In this case, the set $X$ is left empty and $|X|=0 \leq t_{n}$.

- If $|O|=1$, then $t_{n}=0$ and only one output variable $c_{1,2}$ or $c_{2,1}$ is probed. In this case, the probed output variable can be treated as a uniformly random and independent value since the random $r_{1,2}$ is never probed (otherwise we would have $t_{n}>0$ ). Thus, the set $X$ is left empty and $|X|=0 \leq t_{n}$.

Inductive Step: We assume that for $k=j-1$, any set of $t_{j-1}$ intermediate variables and any subset $O_{j-1}$ of outputs can be perfectly simulated from $a_{\mid X_{j-1} \cup O_{j-1}}$ and $b_{\mid X_{j-1} \cup O_{j-1}}$, with $\left|X_{j-1}\right| \leq t_{j-1}$, and we show that any set of $t_{j}$ intermediate variables and any subset $O_{j}$ of outputs can be perfectly simulated from $a_{\mid X_{j} \cup O_{j}}$ and $b_{\mid X_{j} \cup O_{j}}$, with $\left|X_{j}\right| \leq t_{j}$. Let $t^{\prime}$ be the number of internal probes at round $j$, i.e. we have $t_{j}=t_{j-1}+t^{\prime}$.

In the following, we denote by $c_{i}$ the value from previous round $j-1$, i.e. $c_{i, j-1}$ for $i \leq j-2$ and $c_{j-1, j-2}$, and we assume that all probed values $c_{i}$ are considered to be probed at round $j-1$ and not at round $j$. The new $c_{i}$ values from round $j$ will be denoted by $c_{i, j}$. We maintain a list $L$ which is initially filled with all probed intermediate and output variables. The construction of the set $X_{j}$ is performed as follows: initially, we let $X_{j} \leftarrow X_{j-1}$. Then,

- if the variable $c_{j, i}$ for any $i \leq j-1$ is probed, or if $j \in O_{j}$, then we add the index $j$ into $X_{j}$ and one must simulate all intermediate variables $c_{i} \oplus r_{i, j}$ for $i \leq j-1$, i.e. we add in the list $L$ all values $c_{i} \oplus r_{i, j}$ for $i \leq j-1$.

- if two variables among the four variables $\left\{c_{i} \oplus r_{i, j}, a_{i} b_{j} \oplus r_{i, j}, r_{i, j}, c_{i, j}=\left(a_{i} b_{j} \oplus r_{i, j}\right) \oplus a_{j} b_{i}\right\}$ are in the list $L$, i.e. are probed, or must be simulated (because $c_{j, i}$ is probed or $j \in O_{j}$, which leads to the simulation of $c_{i} \oplus r_{i, j}$, or because $i \in O_{j}$ which leads to the simulation of $c_{i, j}$ ), then we add the index $j$ into $X_{j}$ and the index $i$ into $O_{j-1}$.

- Lastly, we add in $X_{j}$ all indices which we added in $O_{j-1}$ but which are not in $O_{j}$.

For the simulation, we start by applying the recurrence assumption which asserts that any set of $t_{j-1}$ intermediate variables and any subset $O_{j-1}$ of outputs at round $j-1$ can be perfectly simulated from $a_{\mid X_{j-1} \cup O_{j-1}}$ and $b_{\mid X_{j-1} \cup O_{j-1}}$, with $\left|X_{j-1}\right| \leq t_{j-1}$. Then, for the simulation of probed variables at round $j$, we consider for all $1 \leq i \leq j-1$, the following three cases:

- if at least two elements among the four variables $\left\{c_{i} \oplus r_{i, j}, a_{i} b_{j} \oplus r_{i, j}, r_{i, j}, c_{i, j}\right\}$ are in the list $L$, then by construction, we have both indices $i$ and $j$ in $X_{j} \cup O_{j}$ and we can perfectly simulate all probed variables at round $j$ with the knowledge of $a_{\mid X_{j} \cup O_{j}}$ and $b_{\mid X_{j} \cup O_{j}}$, and by generating a random for $r_{i, j}$.

- If exactly one element among the four variables $\left\{c_{i} \oplus r_{i, j}, a_{i} b_{j} \oplus r_{i, j}, r_{i, j}, c_{i, j}\right\}$ is in the list $L$, then the random $r_{i, j}$ can play the role of a one-time pad and the probed variable has a uniform distribution and can be simulated by generating a random.

- If $j \in O_{j}$ or one of the $c_{j, i}$ variables for any $i \leq j-1$ is probed, then by construction we know $a_{j}$ and $b_{j}$ since $j \in X_{j}$, and all variables $c_{i} \oplus r_{i, j}$ can be perfectly simulated (since we added them into the list $L$ and already simulated them with the two cases above), therefore we can perfectly simulate the variable $c_{j, i}$ from $a_{\mid X_{j} \cup O_{j}}$ and $b_{\mid X_{j} \cup O_{j}}$.

It remains to show that $\left|X_{j}\right| \leq t_{j}$. Indeed, by recurrence assumption we have $\left|X_{j-1}\right| \leq t_{j-1}$ and since at most one index per probe was added into the set $X_{j}$ at round $j$, we have

$$
\left|X_{j}\right| \leq\left|X_{j-1}\right|+t^{\prime} \leq t_{j-1}+t^{\prime} \leq t_{j} .
$$

As a conclusion, one has proven that the recurrence hypothesis is true for all rounds $k$, and in particular for $k=n$. Thus, by taking $O=O_{n}$ and $X=X_{n}$, one gets that the intermediate and output variables can be perfectly simulated from $a_{\mid X \cup O}$ and $b_{\mid X \cup O}$, with subset $X$ such that $|X| \leq t_{n}$, which terminates the proof. 
Lemma 21 (t-SNI property). We consider Algorithm 6 without lines 11 to 15. We consider an attacker who does not probe the variables $a_{i} \cdot b_{j}$. Consider any set of $t_{n}$ intermediate variables. There exists a set $X$ with $|X| \leq t_{n}+1$ and $n \in X$ such that the $t_{n}$ intermediate variables and $c_{\mid X}$ can be perfectly simulated, while for any $V \subsetneq[1, n] \backslash X$ the variables in $c_{\mid V}$ are uniformly and independently distributed, conditioned on the probed variables and $c_{\mid X}$.

Proof. We prove Lemma 21 by recurrence on the number of input shares $n$. See Figure 11 for an illustration of the proof.

Base Case: The call to Algorithm 6 (without lines 11 to 15) with $n=2$ amounts to performing the following computations: $c_{2,1}=a_{2} b_{2} \oplus\left(a_{1} b_{1} \oplus r_{1,2}\right)$ and $c_{1,2}=\left(a_{1} b_{2} \oplus r_{1,2}\right) \oplus a_{2} b_{1}$. If $t_{n} \geq 1$ we can take $X=[1,2]$ and we can perfectly simulate all variables from the knowledge of the inputs $a_{1}$, $a_{2}, b_{1}$ and $b_{2}$, and we have $|X|=2 \leq t_{n}+1$. Furthermore, the set $V \subsetneq[1,2] \backslash X$ where $X=[1,2]$, is the empty set. If we have $t_{n}=0$, no intermediate variable has been probed. We take $X=\{2\}$ which gives $|X|=1 \leq t_{n}+1$ and $c_{2,1}=a_{2} b_{2} \oplus\left(a_{1} b_{1} \oplus r_{1,2}\right)$ can be perfectly simulated. Indeed since $r_{1,2}$ acts as a one-time pad, the value $\left(a_{1} b_{1} \oplus r_{1,2}\right)$ has a uniform distribution and one then perfectly simulates $c_{2,1}$ with the knowledge of $a_{2} b_{2}$ since $2 \in X$. Then the set $V \subsetneq[1,2] \backslash X=V \subsetneq[1]$ is empty.

Inductive Step: We assume that for $k=j-1$, there exists a set $X_{j-1}$ with $\left|X_{j-1}\right| \leq$ $t_{j-1}+1$ such that the $t_{j-1}$ intermediate variables and $c_{\mid X_{j-1}}$ can be perfectly simulated, while for any $V_{j-1} \subsetneq[1, j-1] \backslash X_{j-1}$ the variables in $c_{\mid V_{j-1}}$ are uniformly and independently distributed, conditioned on the probed variables and $c_{\mid X_{j-1}}$. We show that this is still true for $k=j$.

Let $t^{\prime}$ be the number of internal probes at round $j$, i.e. we have $t_{j}=t_{j-1}+t^{\prime}$. In the following, we denote by $c_{i}$ the value from previous round $j-1$, i.e. $c_{i, j-1}$ for $i \leq j-2$ and $c_{j-1, j-2}$, and we assume that all probed values $c_{i}$ are considered to be probed at round $j-1$ and not at round $j$. The new $c_{i}$ values from round $j$ will be denoted by $c_{i, j}^{\prime}$.

We consider two cases: a first case where none of the intermediate variables $c_{j, i}^{\prime}$ is probed for $1 \leq i<j$, and a second case where at least one variable $c_{j, i}^{\prime}$ is probed.

First case: None of the intermediate variables $c_{j, i}^{\prime}$ is probed. We consider a set $I$ which is constructed as follows:

- if at least one variable among the four variables $\left\{c_{i} \oplus r_{i, j}, a_{i} b_{j} \oplus r_{i, j}, r_{i, j}, c_{i, j}^{\prime}=\left(a_{i} b_{j} \oplus r_{i, j}\right) \oplus a_{j} b_{i}\right\}$ is probed, then we add the index $i$ into $I$.

Then one can apply Lemma 20 with $O=I$ which allows to assert that any set of $t_{j-1}$ intermediate variables and any subset $O$ of outputs at round $j-1$ can be perfectly simulated from $a_{\mid X_{j-1} \cup O}$ and $b_{\mid X_{j-1} \cup O}$, with $\left|X_{j-1}\right| \leq t_{j-1}$. Therefore, the $c_{i}$ variables for $i \in X_{j-1} \cup O$ can be perfectly simulated from $a_{\mid X_{j-1} \cup O}$ and $b_{\mid X_{j-1} \cup O}$ and the variables $c_{i} \oplus r_{i, j}$ and $r_{i, j}$ can be perfectly simulated by generating a random for $r_{i, j}$. Then, one constructs the set $X_{j}$ as follows:

$$
X_{j}=X_{j-1} \cup I \cup\{j\}
$$

and one can perfectly simulate the variables $a_{i} b_{j} \oplus r_{i, j}$ and $c_{i, j}^{\prime}=\left(a_{i} b_{j} \oplus r_{i, j}\right) \oplus a_{j} b_{i}$ from $a_{\mid X_{j}}$ and $b_{\mid X_{j}}$ since $i$ and $j$ are in $X_{j}$. The variable $c_{j, j-1}^{\prime}$ can also be perfectly simulated. Indeed, if $X_{j}=[1, j]$, then one can perfectly simulate it from the knowledge of all input variables $a_{i}$ and $b_{i}$ with $i \leq j$, and otherwise, there exists an index $i^{\star} \notin X_{j}$, which means that the $c_{i^{\star}}$ variable is not probed, nor needed to be simulated (otherwise we would have $i^{\star} \in X_{j}$ ), therefore the variable $r_{i^{\star}, j}$ acts as a one-time pad and the $c_{j, j-1}^{\prime}$ value has a uniform and independent distribution. Thus we have shown that the $t_{j}$ intermediate variables and $c_{\mid X_{j}}^{\prime}$ can be perfectly simulated from $a_{\mid X_{j}}$ and $b_{\mid X_{j}}$.

We note that since $\left|X_{j-1}\right| \leq t_{j-1}$ and $|I| \leq t^{\prime}$, we have as required:

$$
\left|X_{j}\right|=\left|X_{j-1} \cup I \cup\{j\}\right| \leq\left|X_{j-1}\right|+|I|+1 \leq t_{j-1}+t^{\prime}+1 \leq t_{j}+1 .
$$

We now prove that for any $V_{j} \subsetneq[1, j] \backslash X_{j}$ the variables in $c_{\mid V_{j}}^{\prime}$ are uniformly and independently distributed, conditioned on the $t_{j}$ probed variables and $c_{\mid X_{j}}^{\prime}$. 
If $X_{j}=[1, j]$, then the set $V_{j}$ is empty. Otherwise, we consider the previously mentioned index $i^{\star} \notin X_{j}$ and we take without loss of generality $V_{j}=[1, j] \backslash\left(X_{j} \cup\left\{i^{\star}\right\}\right)$.

We first observe that the variable $c_{j, j-1}^{\prime}$ can be rewritten as

$$
c_{j, j-1}^{\prime}=a_{j} b_{j} \oplus \bigoplus_{i=1}^{j-1}\left(c_{i} \oplus r_{i, j}\right)=a_{j} b_{j} \oplus c_{i^{\star}} \oplus r_{i^{\star}, j} \bigoplus_{i=1, i \neq i^{\star}}^{j-1}\left(c_{i} \oplus r_{i, j}\right),
$$

therefore the variable $c_{j, j-1}^{\prime}$ has the same distribution as the random $r_{i^{\star}, j}$, which is uniform and independent, since it does not enter in the computation of any other probed variable. The variable $c_{j, j-1}^{\prime}$ can thus be replaced by a random. As a second step, since the random values $r_{i, j}$ are not used for the simulation of $c_{j, j-1}^{\prime}$ and are only used once for the computation of $c_{i, j}^{\prime}=\left(a_{i} b_{j} \oplus r_{i, j}\right) \oplus a_{j} b_{i}$, the variables $c_{i, j}^{\prime}$ for $i \in V_{j}$ have the same distribution as the variables $r_{i, j}$, which have a uniform and independent distribution. Thus, we have shown that the $c_{i, j}^{\prime}$ variables and the last $c_{j, j-1}^{\prime}$ variable have a uniform and independent distribution. In particular, conditioned on the probed variables and $c_{\mid X_{j}}^{\prime}$ (containing $c_{j, j-1}^{\prime}$ ), the variables in $c_{\mid V_{j}}^{\prime}$ are uniformly and independently distributed.

Second case: At least one of the intermediate variables $c_{j, i}^{\prime}$ is probed. As in the first case, we consider a set $I$ which is constructed as follows: if at least one variable among the four variables $\left\{c_{i} \oplus r_{i, j}, a_{i} b_{j} \oplus r_{i, j}, r_{i, j}, c_{i, j}^{\prime}=\left(a_{i} b_{j} \oplus r_{i, j}\right) \oplus a_{j} b_{i}\right\}$ is probed, then we add the index $i$ into $I$.

Then one can apply the recurrence hypothesis which allows us to perfectly simulate the $t_{j-1}$ intermediate variables and $c_{\mid X_{j-1}}$ at round $j-1$ where $\left|X_{j-1}\right| \leq t_{j-1}+1$, and using both sets $I$ and $X_{j-1}$, one constructs the set $X_{j}$ as before: $X_{j}=X_{j-1} \cup I \cup\{j\}$.

Note that if $X_{j}=[1, j]$, then one can perfectly simulate all variables from the knowledge of all input variables $a_{i}$ and $b_{i}$ with $i \leq j$, and furthermore, the set $V_{j}$ is empty. Otherwise, there exists an index $i^{\star} \notin X_{j}$ and we take without loss of generality $V_{j}=[1, j] \backslash\left(X_{j} \cup\left\{i^{\star}\right\}\right)$.

In the following we show that the $t_{j}$ intermediate variables and $c_{\mid X_{j}}^{\prime}$ can be perfectly simulated. We consider three cases depending on whether the considered index $i \in X_{j}$ is in $X_{j-1}$, in $I \backslash X_{j-1}$, or $i=j$ :

- If $i \in X_{j-1}$ : the $c_{i}$ variables can be perfectly simulated from $a_{\mid X_{j}}$ and $b_{\mid X_{j}}$ thanks to the recurrence assumption (since $X_{j-1} \subset X_{j}$ ) and the variables $c_{i} \oplus r_{i, j}$ and $r_{i, j}$ can be perfectly simulated by generating a random for $r_{i, j}$. Then, since $i$ and $j$ are in $X_{j}$, one can perfectly simulate the variables $a_{i} b_{j} \oplus r_{i, j}$ and $c_{i, j}^{\prime}=\left(a_{i} b_{j} \oplus r_{i, j}\right) \oplus a_{j} b_{i}$ from $a_{\mid X_{j}}$ and $b_{\mid X_{j}}$.

- If $i \in I \backslash X_{j-1}$ : we apply the second part of the recurrence assumption which asserts that for any $V_{j-1} \subsetneq[1, j-1] \backslash X_{j-1}$ the variables in $c_{\mid V_{j-1}}$ are uniformly and independently distributed, conditioned on the probed variables and $c_{\mid X_{j-1}}$. Therefore, we define the set $V_{j-1}=V_{j} \cup$ $\left(I \backslash X_{j-1}\right)$ and the $c_{i}$ variables are uniformly and independently distributed. The variables $c_{i} \oplus r_{i, j}$ and $r_{i, j}$ can be perfectly simulated by generating a random for $r_{i, j}$. Then, since $i$ and $j$ are in $X_{j}$, one can perfectly simulate the variables $a_{i} b_{j} \oplus r_{i, j}$ and $c_{i, j}^{\prime}=\left(a_{i} b_{j} \oplus r_{i, j}\right) \oplus a_{j} b_{i}$ from $a_{\mid X_{j}}$ and $b_{\mid X_{j}}$.

- If $i=j$, then we need to perfectly simulate the variable $c_{j, j-1}^{\prime}$. To this end, we prove that we can perfectly simulate all intermediate variables $c_{i} \oplus r_{i, j}$ for all $i \leq j-1$. We have already proven that is it the case for all $i \in X_{j-1} \cup I$. Since we assume that the index $i^{\star}$ belongs neither to $V_{j}$ nor $X_{j}$, it remains to show that it is still true for $i \in V_{j}$ and for $i=i^{\star}$. If $i=i^{\star}$, then the variables $c_{i^{\star}}$ and $r_{i^{\star}, j}$ are not probed, nor needed to be simulated (otherwise we would have $i^{\star} \in X_{j}$ ), therefore the variable $r_{i^{\star}, j}$ acts as a one-time pad and the $c_{i^{\star}} \oplus r_{i^{\star}, j}$ value has a uniform and independent distribution. If $i \in V_{j}$, we show in the following that we can perfectly simulate the intermediate variables $c_{i} \oplus r_{i, j}$, while proving the second part of the Lemma.

We note that the set $I$ is such that $|I| \leq t^{\prime}-1$ since we are in the case where at least one $c_{j, i}^{\prime}$ is probed and we did not add any index in $I$ for this probed value. Therefore since $\left|X_{j-1}\right| \leq t_{j-1}+1$, we have as required:

$$
\begin{aligned}
\left|X_{j}\right| & =\left|X_{j-1} \cup I \cup\{j\}\right| \leq\left|X_{j-1}\right|+|I|+1 \leq\left(t_{j-1}+1\right)+\left(t^{\prime}-1\right)+1 \\
& \leq t_{j-1}+t^{\prime}+1 \leq t_{j}+1
\end{aligned}
$$


We now prove the second part of the Lemma, i.e. that for any $V_{j} \subsetneq[1, j] \backslash X_{j}$ the variables in $c_{\mid V_{j}}^{\prime}$ are uniformly and independently distributed, conditioned on the $t_{j}$ probed variables and $c_{\mid X_{j}}^{\prime}$.

Since by construction we have defined $V_{j-1}=V_{j} \cup\left(I \backslash X_{j-1}\right)$, we deduce that $V_{j}$ is a subset of $V_{j-1}$, therefore one can apply the recurrence hypothesis which allows us to assert that the $c_{i}$ variables are uniformly and independently distributed. The variables $c_{i} \oplus r_{i, j}$ and $r_{i, j}$ also have a uniform and independent distribution since $r_{i, j}$ is a random value. It remains to show that the variable $c_{i, j}^{\prime}=\left(a_{i} b_{j} \oplus r_{i, j}\right) \oplus a_{j} b_{i}$ is uniformly and independently distributed. This is indeed the case since there is a bijection between $\left(r_{i, j}, c_{i}\right)$ and $\left(c_{i, j}^{\prime}, c_{i} \oplus r_{i, j}\right)$, which means that the distribution of $c_{i, j}^{\prime}$ and $c_{i} \oplus r_{i, j}$ is uniform and independent as it is the case for $r_{i, j}$ and $c_{i}$.

Eventually, as mentioned previously, since $j \in X_{j}$, we need to perfectly simulate the variable $c_{j, j-1}^{\prime}$. This can be done since we have shown that we could perfectly simulate all intermediate variables $\left(c_{i} \oplus r_{i, j}\right)$ for all $i \leq j-1$, and since $j \in X_{j}$ we also know $a_{j} b_{j}$, which allows us to perfectly simulate $c_{j, j-1}^{\prime}=a_{j} b_{j} \oplus \bigoplus_{i=1}^{j-1}\left(c_{i} \oplus r_{i, j}\right)$.

Therefore we have shown that the variables $c_{i, j}^{\prime}$ and $c_{i} \oplus r_{i, j}$ have a uniform and independent distribution, in particular, conditioned on the probed variables and $c_{\mid X_{j}}^{\prime}$ (containing $c_{j, j-1}^{\prime}$ ), the variables in $c_{\mid V_{j}}^{\prime}$ are uniformly and independently distributed, which concludes the proof.

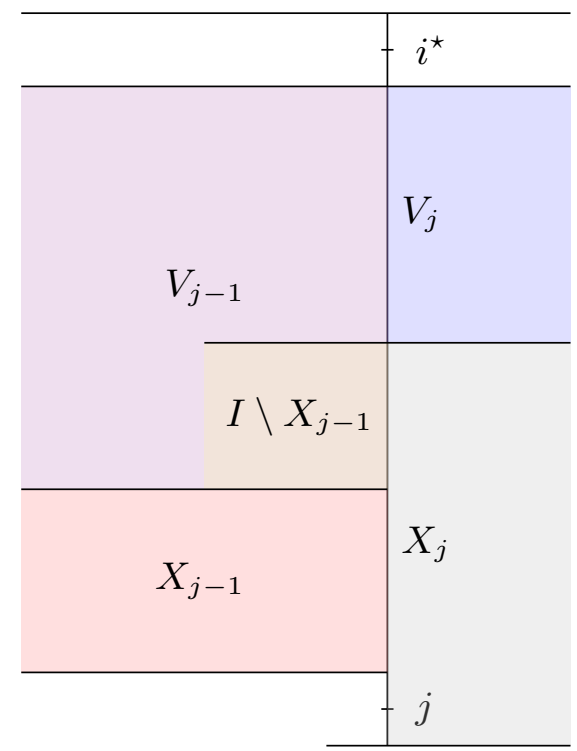

Fig. 11. Illustration of the $t$-SNI sets. Without loss of generality, we can consider any $i^{\star} \in[1, j-1]$ and let $V_{j}=[1, j-1] \backslash\left(X_{j} \cup\left\{i^{\star}\right\}\right)$.

We can now prove Theorem 6.

Proof. We prove Theorem 6 by considering both parts of Algorithm 6 as gadgets; namely, Gadget 1 is the first part of the algorithm (lines 1 to 10) and Gadget 2 is the final loop performing the refresh mask $R$ (lines 11 to 15 ). We show that the composition of Gadget 2 with Gadget 1 is $t$-SNI.

As a first step we assume that intermediate variables $a_{i} b_{j}$ for any $i \neq j$ are not probed and we will consider them at the end of the proof.

Let $t_{n}$ be the number of probes in Gadget 1 and $t^{\prime}$ be the number of internal probes in Gadget 2 , and let $t_{n}^{\prime}$ be the total number of probes in Gadget 1 and 2, i.e. we have $t_{n}^{\prime}=t_{n}+t^{\prime}$. In the following, we denote by $c_{i}$ the values from Gadget 1 , i.e. $c_{i, n}$ for $i \leq n-1$ and $c_{n, n-1}$, and we assume that all probed values $c_{i}$ (including those in Gadget 2) are considered to be probed in Gadget 1 and not in Gadget 2. The new $c_{i}$ values from Gadget 2 will be denoted by $c_{i, j}^{\prime}$. 
In the following, we look at Gadget 2 and we consider two cases: a first case where none of the intermediate variables $c_{n, i}^{\prime}$ (line 13) is probed for $1 \leq i<n$, and a second case where at least one variable $c_{n, i}^{\prime}$ is probed.

First case: None of the intermediate variables $c_{n, i}^{\prime}$ is probed. We consider a set $I^{\prime}$ which is constructed as follows: if at least one variable among the two variables $\left\{c_{i} \oplus s_{i}, s_{i}\right\}$ is probed in Gadget 2 , then we add the index $i$ into $I^{\prime}$.

Then one can apply Lemma 20 with the output set $I^{\prime}$, which allows to assert that the set of $t_{n}$ intermediate variables and any subset $I^{\prime}$ of outputs of Gadget 1 can be perfectly simulated from $a_{\mid X_{n} \cup I^{\prime}}$ and $b_{\mid X_{n} \cup I^{\prime}}$, with $\left|X_{n}\right| \leq t_{n}$. Therefore, the $c_{i}$ variables for $i \in I^{\prime}$ can be perfectly simulated from $a_{\mid X_{n} \cup I^{\prime}}$ and $b_{\mid X_{n} \cup I^{\prime}}$ and the variables $c_{i} \oplus s_{i}$ and $s_{i}$ in Gadget 2 can be perfectly simulated by generating a random for $s_{i}$. Then, one constructs the set $X_{n}^{\prime}$ as follows:

$$
X_{n}^{\prime}=X_{n} \cup I^{\prime} .
$$

We note that since $\left|X_{n}\right| \leq t_{n}$ and $\left|I^{\prime}\right| \leq t^{\prime}$, we have as required:

$$
\left|X_{n}^{\prime}\right|=\left|X_{n} \cup I^{\prime}\right| \leq\left|X_{n}\right|+\left|I^{\prime}\right| \leq t_{n}+t^{\prime} \leq t_{n}^{\prime} .
$$

Furthermore, since $\left|X_{n}^{\prime}\right| \leq t_{n}^{\prime}$ and since by definition of Theorem 6 we have $t_{n}^{\prime}+|O|<n$, we deduce that $\left|X_{n}^{\prime} \cup O\right| \leq\left|X_{n}^{\prime}\right|+|O| \leq t_{n}^{\prime}+|O|<n$. Thus, there exists an index $i^{\star} \notin X_{n}^{\prime} \cup O$ and two cases arise:

- If $i^{\star}=n$ then $n \notin O$ which means that $c_{n, n-1}^{\prime}$ need not be simulated. Furthermore, all output variables $c_{i}^{\prime}=s_{i}$ with $i \in O$ can also be simulated by generating a random.

- If $i^{\star} \neq n$, this means that the variables $c_{i^{\star}}, c_{i^{\star}} \oplus s_{i^{\star}}$ and $s_{i^{\star}}$ are not probed nor needed to be simulated. Thus, we first observe that the variable $c_{n, n-1}^{\prime}$ can be rewritten as

$$
c_{n, n-1}^{\prime}=c_{n} \oplus \bigoplus_{i=1}^{n-1}\left(c_{i} \oplus s_{i}\right)=c_{n} \oplus c_{i^{\star}} \oplus s_{i^{\star}} \bigoplus_{i=1, i \neq i^{\star}}^{n-1}\left(c_{i} \oplus s_{i}\right),
$$

therefore the variable $c_{n, n-1}^{\prime}$ has the same distribution as the random $s_{i^{\star}}$, which is uniform and independent, since it does not enter in the computation of any other probed variable. The variable $c_{n, n-1}^{\prime}$ can thus be replaced by a random. As a second step, since the random values $s_{i}$ are not used for the simulation of $c_{n, n-1}^{\prime}$ and are only used once for the output $c_{i}^{\prime}=s_{i}$, the variables $c_{i}^{\prime}$ for $i \in O$ have the same distribution as the variables $s_{i}$, which have a uniform and independent distribution and can thus be perfectly simulated by generating a random.

As a conclusion, we have shown that in the case where none of the intermediate variables $c_{n, i}^{\prime}$ is probed, the $t_{n}^{\prime}$ intermediate variables and $c_{\mid O}^{\prime}$ can be perfectly simulated from $a_{\mid X_{n}^{\prime}}$ and $b_{\mid X_{n}^{\prime}}$ with $\left|X_{n}^{\prime}\right| \leq t_{n}^{\prime}$

Second case: At least one of the intermediate variables $c_{n, i}^{\prime}$ is probed. As in the first case, we consider a set $I^{\prime}$ which is constructed as follows: if at least one variable among the two variables $\left\{c_{i} \oplus s_{i}, s_{i}\right\}$ is probed in Gadget 2, then we add the index $i$ into $I^{\prime}$.

Then one can apply Lemma 21 on Gadget 1, namely, for $j=n$ and $i=n-1$ (i.e. at the end of Gadget 1), there exists a set $X_{n}$ with $\left|X_{n}\right| \leq t_{n}+1$ and $n \in X_{n}$ such that the $t_{n}$ intermediate variables probed in Gadget 1 and the output values $c_{\mid X_{n}}$ from Gadget 1 can be perfectly simulated from $a_{\mid X_{n}}$ and $b_{\mid X_{n}}$, while for any $V_{n} \subsetneq[1, n] \backslash X_{n}$ the variables in $c_{\mid V_{n}}$ are uniformly and independently distributed, conditioned on the probed variables and $c_{\mid X_{n}}$. Using both sets $I^{\prime}$ and $X_{n}$, one constructs the set $X_{n}^{\prime}$ as before: $X_{n}^{\prime}=X_{n} \cup I^{\prime}$.

We note that the set $I^{\prime}$ is such that $\left|I^{\prime}\right| \leq t^{\prime}-1$ since we are in the case where at least one $c_{n, i}^{\prime}$ is probed and we did not add any index in $I^{\prime}$ for this probed value. Therefore since $\left|X_{n}\right| \leq t_{n}+1$, we have as required:

$$
\left|X_{n}^{\prime}\right|=\left|X_{n} \cup I^{\prime}\right| \leq\left|X_{n}\right|+\left|I^{\prime}\right| \leq\left(t_{n}+1\right)+\left(t^{\prime}-1\right) \leq t_{n}+t^{\prime} \leq t_{n}^{\prime} .
$$




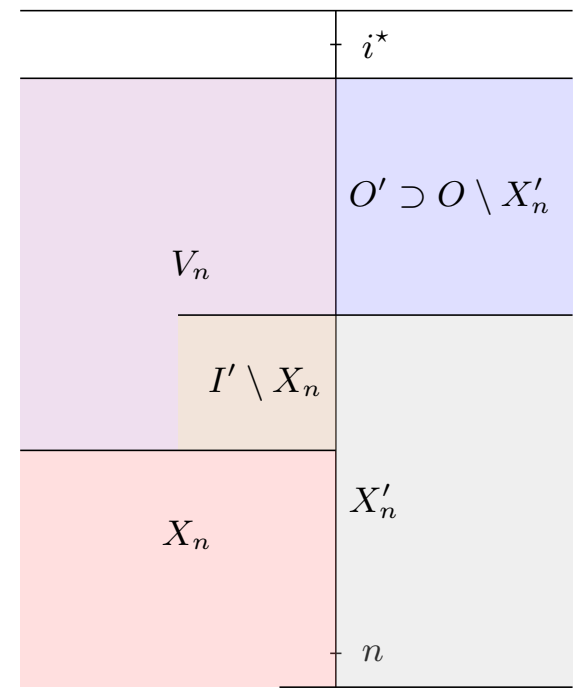

Fig. 12. Illustration of the $t$-SNI sets for the second case of Theorem 6 .

Furthermore, since $\left|X_{n}^{\prime}\right| \leq t_{n}^{\prime}$ and since by definition of Theorem 6 we have $t_{n}^{\prime}+|O|<n$, we deduce that $\left|X_{n}^{\prime} \cup O\right| \leq\left|X_{n}^{\prime}\right|+|O| \leq t_{n}^{\prime}+|O|<n$. Thus, there exists an index $i^{\star} \notin X_{n}^{\prime} \cup O$ and we consider the set $O^{\prime}=[1, n] \backslash\left(X_{n}^{\prime} \cup\left\{i^{\star}\right\}\right)$ which includes the set $O \backslash X_{n}^{\prime}$, that is we have $O \backslash X_{n}^{\prime} \subset O^{\prime}$.

In the following we show that the $t_{n}^{\prime}$ intermediate variables of gadgets 1 and 2 including $c_{\mid X_{n}^{\prime}}^{\prime}$, and the output variables $c_{\mid O}^{\prime}$ can be perfectly simulated from $a_{\mid X_{n}^{\prime}}$ and $b_{\mid X_{n}^{\prime}}$.

Since $n \in X_{n}$ from Lemma 21, we will need to perfectly simulate the variable $c_{n, n-1}^{\prime}$. To this end, we prove that we can perfectly simulate all intermediate variables $c_{i} \oplus s_{i}$ for all $i \leq n-1$, which will allow us to also perfectly simulate all probed intermediate variables $c_{n, i}^{\prime}$, including the last one $c_{n, n-1}^{\prime}$.

Since we assume that the index $i^{\star}$ belongs neither to $O^{\prime}$ nor $X_{n}^{\prime}$, and since we have $X_{n}^{\prime}=X_{n} \cup I^{\prime}$, we consider four cases depending on whether the considered index $i$ is in $X_{n}$, in $I^{\prime} \backslash X_{n}$, in $O^{\prime}$ or $i=i^{\star}$ :

- If $i \in X_{n}$ : the $c_{i}$ variables in Gadget 1 can be perfectly simulated from $a_{\mid X_{n}^{\prime}}$ and $b_{\mid X_{n}^{\prime}}$ thanks to Lemma 21 (since $X_{n} \subset X_{n}^{\prime}$ ) and the variables $c_{i} \oplus s_{i}$ and $s_{i}$ can be perfectly simulated by generating a random for $s_{i}$.

- If $i \in I^{\prime} \backslash X_{n}$ : we apply the second part of Lemma 21 which asserts that for any $V_{n} \subsetneq[1, n] \backslash X_{n}$ the variables in $c_{\mid V_{n}}$ in Gadget 1 are uniformly and independently distributed, conditioned on the probed variables and $c_{\mid X_{n}}$. Therefore, we define the set $V_{n}=O^{\prime} \cup\left(I^{\prime} \backslash X_{n}\right)$ and the $c_{i}$ variables are uniformly and independently distributed. The variables $c_{i} \oplus s_{i}$ and $s_{i}$ can be perfectly simulated by generating a random for $s_{i}$.

- If $i=i^{\star}$ (we note that we have $i^{\star} \neq n$ since $n \in X_{n}$ ), then the variables $c_{i^{\star}}$ and $s_{i^{\star}}$ are not probed, nor needed to be simulated (otherwise we would have $i^{\star} \in X_{n}^{\prime}$ ), therefore the variable $s_{i^{\star}}$ acts as a one-time pad and the $c_{i^{\star}} \oplus s_{i^{\star}}$ value has a uniform and independent distribution.

- If $i \in O^{\prime}$, since by construction we have defined $V_{n}=O^{\prime} \cup\left(I^{\prime} \backslash X_{n}\right)$, we deduce that $O^{\prime}$ is a subset of $V_{n}$, therefore one can apply Lemma 21 on Gadget 1 which allows us to assert that the $c_{i}$ variables are uniformly and independently distributed and can thus be perfectly simulated by generating a random value for $c_{i}$. As a consequence, one can also perfectly simulate the variables $c_{i} \oplus s_{i}$ and $c_{i}^{\prime}=s_{i}$ by generating a random value for $s_{i}$.

Eventually, the simulation of the variable $c_{n, n-1}^{\prime}$ can be done since we have shown that we could perfectly simulate all intermediate variables $c_{i} \oplus s_{i}$ for all $i \leq n-1$, and since $n \in X_{n}$ we can also simulate $c_{n}$, which allows us to perfectly simulate $c_{n, n-1}^{\prime}=c_{n} \oplus \bigoplus_{i=1}^{n-1}\left(c_{i} \oplus s_{i}\right)$.

Therefore we have shown that if at least one of the intermediate variables $c_{n, i}^{\prime}$ is probed, then the $t_{n}^{\prime}$ intermediate variables of gadgets 1 and 2 including $c_{\mid X_{n}^{\prime}}^{\prime}$ can be perfectly simulated from 
$a_{\mid X_{n}^{\prime}}$ and $b_{\mid X_{n}^{\prime}}$ with $\left|X_{n}^{\prime}\right| \leq t_{n}^{\prime}$. We have also shown that the output variables $c_{\mid O^{\prime}}^{\prime}$, which includes the variables $c_{\mid O \backslash X_{n}^{\prime}}^{\prime}$ (since we have $O \backslash X_{n}^{\prime} \subset O^{\prime}$ ), are uniformly and independently distributed.

It remains to deal with the intermediate variables $a_{i} b_{j}$ for $i \neq j$. Let $t_{a b}$ be the number of such probes. We construct the sets $I$ and $J$ as follows: $I=X_{n}^{\prime}$ and $J=X_{n}^{\prime}$, and for all such probed variables $a_{i} b_{j}$, we add $i$ into $I$ and $j$ into $J$. The variables $a_{i} b_{j}$ can trivially be perfectly simulated from $a_{\mid I}$ and $b_{\mid J}$. Furthermore, since we have shown that all other $t_{n}^{\prime}$ probed intermediate variables and $c_{\mid O}$ can be perfectly simulated from $a_{\mid X_{n}^{\prime}}$ and $b_{\mid X_{n}^{\prime}}$, and since $X_{n}^{\prime} \subset I$ and $X_{n}^{\prime} \subset J$, we deduce that the total $t=t_{n}^{\prime}+t_{a b}$ internal probed variables and $c_{\mid O}$ can be perfectly simulated from $a_{\mid I}$ and $b_{\mid J}$. Eventually, since

$$
|I|=|J|=\left|X_{n}^{\prime}\right|+t_{a b} \leq t_{n}^{\prime}+t_{a b} \leq t
$$

we get the required bounds $|I| \leq t$ and $|J| \leq t$.

Thus, the composition of Gadget 2 with Gadget 1 is $t$-SNI which concludes the proof.

\section{Private Circuits with Multiple PRGs without Robustness}

\section{D.1 Proof of Proposition 4 (Security of PINI-R)}

Let $t_{1}$ be the number of probes on intermediate variables and let $t_{2}$ be the number of extended probes on the randomness subsets $\rho_{i}$, with $t_{1}+t_{2}<n$. From the PINI-R property, those $t_{1}+t_{2}$ probes can be simulated from a subset $I \cup R$ of input shares, with $|I| \leq t_{1}$ and $|R| \leq t_{2}$, which gives $|I \cup R| \leq t_{1}+t_{2}<n$. Since any proper subset of the input shares is uniformly and independently distributed, the adversary learns nothing from the secret variables of the original circuit.

\section{D.2 Proof of Theorem 8 (Composition of PINI-R)}

The proof is essentially the same as in [CS18]. We consider $l$ Gadgets $G_{1}, \cdots, G_{l}$ which are PINI-R, that we order as a direct acyclic graph from output to input in a reverse topological sort order. We assume that each gadget $G_{k}$ has $t_{k}$ internal probes and that the sum of all internal probes is equal to $t$. We will also denote by $\rho_{i}^{(k)}$ the randoms for $1 \leq i \leq n$ in Gadget $G_{k}$. Furthermore, we must simulate the output probes in $\mathcal{O}$ from the last gadget $G_{1}$. We prove by recurrence on $k$ that the composition of PINI-R gadgets is PINI-R.

If $k=1$, then there is only one gadget and this is straightforward since by assumption the gadget is PINI-R. Now we assume that the composition of gadgets $G_{1}, \cdots, G_{k}$ is PINI-R and we prove that the composition of gadgets $G_{1}, \cdots, G_{k+1}$ is still PINI-R.

Since the composition of gadgets $G_{1}, \cdots, G_{k}$ is PINI-R, we get that for any set of $t_{1}+\cdots+t_{k}$ intermediate variables, any subset $R_{k} \subset[1, n]$ of random indices and any subset $\mathcal{O}$ of output indices, there exists a subset $I_{k} \subset[1, n]$ of input indices with $\left|I_{k}\right| \leq t_{1}+\cdots+t_{k}$ such that the $t_{1}+\cdots+t_{k}$ intermediate variables, the randoms $\tilde{\rho}_{i}=\bigcup_{j=1}^{k} \rho_{i}^{(j)}$ for $i \in R_{k}$ and the output shares $y_{\mid \mathcal{O} \cup R_{k}, \star}$ can be perfectly simulated from the input shares $x_{\mid I_{k} \cup \mathcal{O} \cup R_{k}, \star} \cdot$

The output shares of $G_{k+1}$ are the input shares of the composite gadget $G_{1}, \cdots, G_{k}$. Since gadget $G_{k+1}$ is PINI-R, for any $R_{k+1}$, one can simulate the $t_{k+1}$ probes within Gadget $G_{k+1}$, the randoms $\rho_{i}^{(k+1)}$ for $i \in R_{k+1}$ and the output shares $y_{\mid\left(I_{k} \cup \mathcal{O} \cup R_{k}\right) \cup R_{k+1}, \star}$ with input shares corresponding to indices in $S_{k+1} \cup R_{k+1} \cup\left(I_{k} \cup \mathcal{O} \cup R_{k}\right)$ with $\left|S_{k+1}\right| \leq t_{k+1}$.

The PINI-R property must hold for any subset $R$. We take $R_{k+1}=R_{k}=R$. Therefore we can compose these simulators to perfectly simulate the composition of gadgets $G_{1}, \cdots, G_{k+1}$ (namely, the $t_{1}+\cdots+t_{k+1}$ intermediate variables, the randoms $\hat{\rho}_{i}=\bigcup_{j=1}^{k+1} \rho_{i}^{(j)}$ for $i \in R$ and the output shares $\left.y_{\mid \mathcal{O} \cup R, \star}\right)$ from the input shares with indices in $I_{k+1} \cup \mathcal{O} \cup R$ with $I_{k+1}=S_{k+1} \cup I_{k}$. We get as required

$$
\left|I_{k+1}\right|=\left|S_{k+1} \cup I_{k}\right| \leq\left|S_{k+1}\right|+\left|I_{k}\right| \leq t_{k+1}+\left(t_{1}+\cdots+t_{k}\right) \leq t
$$

which allows us to conclude that the composition of $k+1$ gadgets PINI-R remains PINI-R. 
For $k+1=l$, we get that we can perfectly simulate the composition of gadgets $G_{1}, \cdots, G_{l}$ from the input shares with indices in $I_{l} \cup \mathcal{O} \cup R$ with $\left|I_{l}\right|=\left|\bigcup_{k=1}^{l} S_{k}\right| \leq t_{1}+\cdots+t_{l} \leq t$. This terminates the proof.

\section{D.3 Security under the PINI-R and $t$-SNI-R notions}

Lemma 22 (PINI-R of LR). The LR gadget is PINI-R.

Proof. The proof is straightforward from the PINI property of LR. Namely it suffices to take $O^{\prime}=O \cup R$.

For simplicity we prove the $t$-SNI-R property of SecMult and SecMultILR with $t=n-1$, since this implies the $t$-SNI-R property for any $1 \leq t \leq n-1$.

Lemma 23 ( $t$-SNI-R of SecMult). We consider the SecMult construction. Consider any set of $t$ intermediate variables, any subset $O \subset[1, n]$ of output variables, and any subset $R \subset[1, n-1]$, such that $t+|O|+|R|<n$. Then the $t$ intermediate variables, the output variables $c_{\mid O \cup R}$, and all randoms $r_{i, j}$ for $i \in R$ can be perfectly simulated from the knowledge of $a_{\mid I \cup R}$ and $b_{\mid J \cup R}$, with $|I| \leq t$ and $|J| \leq t$.

Proof. The proof is similar to the $t$-SNI proof of SecMult in $\left[\mathrm{BBD}^{+} 16\right]$. We construct two sets $I$ and $J$ corresponding to the input shares of $a$ and $b$ respectively. We divide the internal probes in 4 groups. The four groups are processed separately and sequentially, that is we start with all probes in Group 1, and finish with all probes in Group 4.

- Group 1: If $a_{i}, b_{i}$ or $a_{i} \cdot b_{i}$ is probed, add $i$ to $I$ and $J$.

- Group 2: If $r_{i, j}$ or $c_{i, j}$ is probed (for any $i \neq j$ ), add $i$ to $I$ and $J$.

Note that after the processing of Group 1 and 2 probes, we have $I=J$; we denote by $U$ the common value of $I$ and $J$ after the processing of Group 1 and 2 probes, to which we add the set $R$, that is we have $U=I \cup R=J \cup R$.

- Group 3: If $a_{i} \cdot b_{j} \oplus r_{i, j}$ is probed: if $i \in U$ or $j \in U$, add $\{i, j\}$ to both $I$ and $J$.

- Group 4: If $a_{i} \cdot b_{j}$ is probed (for any $i \neq j$ ), then add $i$ to $I$ and $j$ to $J$.

We have $|I| \leq t$ and $|J| \leq t$, since for every probe we add at most one index in $I$ and $J$. The simulation of probed variables in groups 1 and 4 is straightforward. Note that for $i<j$, the variable $r_{i j}$ is used in all partial sums $c_{i k}$ for $k \geq j$; moreover $r_{i j}$ is used in $r_{i j} \oplus a_{i} b_{j}$, which is used in $r_{j i}$, which is used in all partial sums $c_{j k}$ for $k \geq i$. Therefore if $i \notin U$, then $r_{i j}$ is not probed and does not enter in the computation of any probed $c_{i k}$; symmetrically if $j \notin U$, then $r_{j i}$ is not probed and does not enter in the computation of any probed $c_{j k}$.

For any pair $i<j$, we can now distinguish 4 cases:

- Case 1: $\{i, j\} \in U$. In that case, we can perfectly simulate all variables $r_{i j}, a_{i} \cdot b_{j}, a_{i} \cdot b_{j} \oplus r_{i j}$, $a_{j} \cdot b_{i}$ and $r_{j i}$. In particular, we let $r_{i j} \leftarrow \mathbb{F}_{2^{k}}$, as in the real circuit.

- Case 2: $i \in U$ and $j \notin U$. In that case we simulate $r_{i j} \leftarrow \mathbb{F}_{2^{k}}$, as in the real circuit. If $a_{i} \cdot b_{j} \oplus r_{i, j}$ is probed (Group 3), we can also simulate it since $i \in U$ and $j \in J$ by definition of the processing of Group 3 variables.

- Case 3: $i \notin U$ and $j \in U$. In that case $r_{i j}$ has not been probed, nor any variable $c_{i k}$, since otherwise $i \in U$. Therefore $r_{i j}$ is not used in the computation of any probed variable (except $r_{j i}$, and possibly $\left.a_{i} \cdot b_{j} \oplus r_{i, j}\right)$. Therefore we can simulate $r_{j i} \leftarrow \mathbb{F}_{2^{k}}$; moreover if $a_{i} \cdot b_{j} \oplus r_{i j}$ is probed, we can perfectly simulate it using $a_{i} \cdot b_{j} \oplus r_{i j}=a_{j} \cdot b_{i} \oplus r_{j i}$, since $j \in U$ and $i \in J$ by definition of the processing of Group 3 variables. 
- Case 4: $i \notin U$ and $j \notin U$. If $a_{i} b_{j} \oplus r_{i, j}$ is probed, since $r_{i j}$ is not probed and does not enter into the computation of any other probed variable, we can perfectly simulate such probe with a random value.

From cases 1, 2 and 3, we obtain that for any $i \neq j$, we can perfectly simulate any variable $r_{i j}$ such that $i \in U$. This implies that we can also perfectly simulate all partial sums $c_{i k}$ when $i \in U$, including the output variables $c_{i}$. Finally, all probed variables and all randoms $r_{i, j}$ for $i \in R$ are perfectly simulated from the knowledge of $a_{\mid I \cup R}$ and $b_{\mid J \cup R}$.

We now consider the simulation of the output variables $c_{i}$. We must show how to simulate $c_{i}$ for all $i \in \mathcal{O} \cup R$, where $\mathcal{O}$ is an arbitrary subset of $[1, n]$ and $R$ is an arbitrary subset of $[1, n-1]$, such that $t+|O|+|R|<n$. For $i \in U \supset R$, such variables are already perfectly simulated, as explained above. We now consider the output variables $c_{i}$ with $i \notin U$. We construct a subset of indices $V$ as follows: for any probed Group 3 variable $a_{i} b_{j} \oplus r_{i j}$ such that $i \notin U$ and $j \notin U$ (this corresponds to Case 4), we put $j$ in $V$ if $i \in \mathcal{O}$, otherwise we put $i$ in $V$. Since we have only considered Group 3 probes, we must have $|U|+|V| \leq t$, which implies $|U|+|V|+|\mathcal{O}|<n$. Therefore there exists an index $j^{\star} \in[1, n]$ such that $j^{\star} \notin U \cup V \cup \mathcal{O}$. For any $i \in \mathcal{O}$, we can write:

$$
c_{i}=a_{i} b_{i} \oplus \bigoplus_{j \neq i} r_{i j}=r_{i, j^{\star}} \oplus\left(a_{i} b_{i} \oplus \bigoplus_{j \neq i, j^{\star}} r_{i j}\right)
$$

We claim that neither $r_{i, j^{\star}}$ nor $r_{j^{\star}, i}$ do enter into the computation of any probed variable or other $c_{i^{\prime}}$ for $i^{\prime} \in \mathcal{O}$. Namely $i \notin U$ so neither $r_{i, j^{\star}}$ nor any partial sum $c_{i k}$ was probed; similarly $j^{\star} \notin U$ so neither $r_{j^{\star}, i}$ nor any partial sum $c_{j^{\star}, k}$ was probed, and the output $c_{j^{\star}}$ does not have to be simulated since by definition $j^{\star} \notin \mathcal{O}$. Finally if $i<j^{\star}$ then $a_{i} b_{j^{\star}} \oplus r_{i, j^{\star}}$ was not probed since otherwise $j^{\star} \in V$ (since $i \in \mathcal{O}$ ); similarly if $j^{\star}<i$ then $a_{j^{\star}} b_{i} \oplus r_{j^{\star}, i}$ was not probed since otherwise we would have $j^{\star} \in V$ since $j^{\star} \notin \mathcal{O}$. Therefore, since neither $r_{i, j^{\star}}$ nor $r_{j^{\star}, i}$ are used elsewhere, we can perfectly simulate $c_{i}$ by generating a random value. Thus, we have shown that the output variables $c_{\mid O \cup R}$ can be perfectly simulated from the knowledge of $a_{\mid I \cup R}$ and $b_{\mid J \cup R}$. This terminates the proof of Lemma 23.

Lemma 24 ( $t$-SNI-R of SecMultILR). We consider the SecMultILR construction. Consider any set of $t$ intermediate variables, any subset $O \subset[1, n]$ of output variables, and any subset $R \subset$ $[1, n-1]$, such that $t+|O|+|R|<n$. Then the $t$ intermediate variables, the output variables $c_{\mid O \cup R}$, and all randoms $r_{i, j}$ and $s_{i, j}$ for $i \in R$ can be perfectly simulated from the knowledge of $a_{\mid I \cup R}$ and $b_{\mid J \cup R}$, with $|I| \leq t$ and $|J| \leq t$.

Proof. The proof is essentially the same as for Theorem 4 . We proceed with a sequence of games. In Game 0, the variables are simulated as in the original circuit. In Game 1, we modify the distribution of the variables $s_{i j}$ so that the circuit being computed with the new $s_{i j}^{\prime}$ variables corresponds to the mask refreshing of [RP10]. In Game 2, we rewrite the distribution of each intermediate variable as the xor of a function $f$ corresponding to all $s_{i j}^{\prime}=0$, and a function $g$ of the $s_{i j}^{\prime}$ variables only. Finally, in Game 3, we argue that the $f$ function corresponds to the simulation of the original SecMult circuit, with the $t$-SNI-R property, as proved in Theorem 23, while the $g$ function can be simulated directly from the random variables $s_{i j}^{\prime}$; this terminates the proof.

\section{D.4 Proof of Theorem 9 (Locality composition with randomness subset)}

In the composite gadget $\mathcal{G}$, each gadget $G_{k}^{\prime}$ takes as input $a_{i}$ and $b_{i}$ which are locality refreshed from previous gadgets $G_{k_{1}}^{\prime}$ and $G_{k_{2}}^{\prime}$ with randoms $s_{i}^{\left(k_{1}\right)}$ and $s_{i}^{\left(k_{2}\right)}$ for $1 \leq i \leq n-1$. Within the $G_{k}$ gadget, the locality is equal to $\ell$ with regards to the randomness $\rho_{k}$; since the output of $G_{k_{1}}^{\prime}$ and $G_{k_{2}}^{\prime}$ are locality refreshed, the intermediate variables do not depend on any other $\rho_{u}$ for $u \neq k$. Moreover, within the $G_{k}$ gadget, the locality of variables is equal to 2 with respect to the randoms $s_{i}^{(u)}$ for a fixed $i$; namely the inputs $a_{i}$ and $b_{i}$ depend on the randoms $s_{i}^{\left(k_{1}\right)}$ and $s_{i}^{\left(k_{2}\right)}$ only. 
Eventually, a mask refreshing is applied on the output of $G_{k}$, which implies that each intermediate variable from the mask refreshing can depend on at most 3 of the randoms $s_{i}^{\left(k_{1}\right)}, s_{i}^{\left(k_{2}\right)}$ and $s_{i}^{(k)}$ for a given $i$. Therefore we have shown that in the global construction $\mathcal{G}$, the locality of variables is equal to $\ell$ with regards to the set of randoms $\left\{\rho_{u}: u \in K\right\}$, and 3 with respect to the set of randoms $\left\{s_{i}^{(u)}: u \in K\right\}$.

\section{D.5 Locality $\ell=1$ with respect to randomness subsets}

Theorem 13. Let $G_{k}$ for $k \in K$ be a set of 2-input gadgets with locality refreshed output with randoms $s_{i}^{c,(k)}$ for $1 \leq i \leq n-1$, which all make an $\ell$-local use of their randomness $\rho_{k}$. Let $\tilde{G}_{k}$ be the same gadgets where the 2 inputs of each gadget $G_{k}$ is locality refreshed with randoms $s_{i}^{a,(k)}$ and $s_{i}^{b,(k)}$ for $1 \leq i \leq n-1$. Any composite gadget made of $\tilde{G}_{k}$ makes an $\ell$-local use of the randomness $\left(\rho_{k}\right)_{k \in K}$, and for any $1 \leq i \leq n-1$, it makes a 1-local use of the randoms in each of the following sets $\left\{s_{i}^{a,(k)}, k \in K\right\},\left\{s_{i}^{b,(k)}, k \in K\right\}$ and $\left\{s_{i}^{c,(k)}, k \in K\right\}$.

Proof. In the composite gadget $\mathcal{G}$, each gadget $\tilde{G}_{k}$ takes as input $a_{i}$ and $b_{i}$ which are locality refreshed from previous gadgets $k_{1}$ and $k_{2}$ with randoms $s_{i}^{c,\left(k_{1}\right)}$ and $s_{i}^{c,\left(k_{2}\right)}$. The inputs $a_{i}$ are then locality refreshed in algorithms $\mathrm{LR}_{A}$ and $\mathrm{LR}_{B}$ with randoms $s_{i}^{a,(k)}$ and $s_{i}^{b,(k)}$ respectively.

Within the gadget $\mathrm{LR}_{A}$, the variables have a locality of 1 regarding the sets of randoms $\left\{s_{i}^{a,(k)}, k \in K\right\}$ and $\left\{s_{i}^{c,(k)}, k \in K\right\}$. Similarly, within the gadget $\mathrm{LR}_{B}$, the locality regarding the sets of randoms $\left\{s_{i}^{b,(k)}, k \in K\right\}$ and $\left\{s_{i}^{c,(k)}, k \in K\right\}$ is 1 . By construction, the outputs $a_{i}^{\prime}$ and $b_{i}^{\prime}$ of gadgets $\mathrm{LR}_{A}$ and $\mathrm{LR}_{B}$ do not depend anymore on randoms of type $C$. Therefore the input shares $a_{i}^{\prime}$ and $b_{i}^{\prime}$ of the $G_{k}$ gadget have a locality of 1 regarding the sets of randoms $\left\{s_{i}^{a,(k)}, k \in K\right\}$ and $\left\{s_{i}^{b,(k)}, k \in K\right\}$, and they do not depend on randoms of type $C$.

Within the $G_{k}$ gadget, the locality of variables is equal to $\ell$ with regards to the randomness $\rho_{k}$, and equal to 1 with respect to $s_{i}^{a,(k)}$ and $s_{i}^{b,(k)}$ for any $1 \leq i \leq n-1$. Eventually, a mask refreshing $\mathrm{LR}_{C}$ is applied, using randoms $s_{i}^{c,(k)}$. Within Gadget $\mathrm{LR}_{C}$, by definition the locality of variables is equal to $\ell$ with regards the randomness in $\rho_{k}$, and equal to 1 with respect to the randoms $s_{i}^{a,(k)}$, $s_{i}^{b,(k)}$ and $s_{i}^{c,(k)}$. At the end of Gadget $\mathrm{LR}_{C}$ the dependence to randoms from $\rho_{k}, s_{i}^{a,(k)}$ and $s_{i}^{b,(k)}$ disappears, thus the only remaining dependence is the one regarding the random $s_{i}^{c,(k)}$, so with locality 1.

Therefore we have shown that in the global construction $\mathcal{G}$, the locality of variables is equal to $\ell$ with regards to sets of randoms $\left(\rho_{k}\right)_{k \in K}$, and 1 with respect to the set of randoms $\left\{s_{i}^{a,(k)}, k \in K\right\}$, $\left\{s_{i}^{b,(k)}, k \in K\right\}$ and $\left\{s_{i}^{c,(k)}, k \in K\right\}$.

\section{D.6 Proof of Lemma 13 (locality of SecMultILR)}

Thanks to the loop from Line 11 to 15 of SecMultILR performing at each step $j$ a locality refresh of the shares $\left(c_{1}, \ldots, c_{j}\right)$, no intermediate variable can depend on two randoms $r_{i j}$ on the same row $i$; therefore the SecMultILR algorithm makes a 1-local use of each randomness set $\rho_{i}=\left\{r_{i j}\right.$ : $i \leq j \leq n\}$. Similarly, at step $j$ the intermediate variables can only depend on randoms $s_{i, j-1}$ and $s_{i, j}$ for a fixed $i$. Therefore the SecMultILR algorithm makes a 2-local use of each randomness set $\rho_{i}^{\prime}=\left\{s_{i j}: i \leq j \leq n\right\}$.

\section{E Implementation of AES}

\section{E.1 Working over $\mathbb{F}_{2^{16}}$}

For the PRG based on polynomial evaluation we need to work over $\mathbb{F}_{2^{16}}$, which is a degree-2 extension of $\mathbb{F}_{2^{8}}$. This means that any element of $\mathbb{F}_{2^{16}}$ can be represented as $a_{0}+a_{1} \cdot z$ where 
$a_{0}, a_{1} \in \mathbb{F}_{2^{8}}$. The polynomial $f(z)=z^{2}+z+t^{5}$ is irreducible over $\mathbb{F}_{2^{8}}$. Working modulo $f(z)$, we have:

$$
\begin{aligned}
\left(a_{0}+a_{1} \cdot z\right) \cdot\left(b_{0}+b_{1} \cdot z\right) & =a_{0} b_{0}+z\left(a_{1} b_{0}+a_{0} b_{1}\right)+a_{1} b_{1} z^{2} \\
& =a_{0} b_{0}+t^{5} a_{1} b_{1}+z\left(a_{1} b_{0}+a_{0} b_{1}+a_{1} b_{1}\right)
\end{aligned}
$$

\section{E.2 Implementation of AES with multiple PRGs, SecMultFLR and 3-wise independent PRG}

We perform a locality refresh of the 2 inputs of each gadget (with two distinct sets of independent PRGs), and we perform a locality refresh of the outputs of each gadget (including each Xor), using another distinct set of independent PRGs. In that case, the locality with respect to each subset of randoms is always $\ell=1$; therefore, we can use a PRG with $r$-wise independence $r=t=n-1$. With our 3 -wise independent PRG function $G$, this implies that this specific PRG only works for $n=3$ and $n=4$ shares.

There are therefore 4 classes of independent PRGs. The first class generates the randoms $r_{i j}$ of the SecMult gadgets, with a dedicated PRG for each $r_{i j}$. The other 3 classes generate the randoms $s_{i}$ for the locality refresh of the 2 inputs and output of each gadget. We must therefore determine the number of SecMult gadgets (including the FullRefresh gadget), and the total number of gadgets.

As previously the number of SecMult gadgets (including the FullRefresh gadgets) is 960. Moreover, each MixColumns comprises 48 xors, for the 9 first rounds of the AES (the 10th round does not have a MixColumns operation). This gives a total of $9 \cdot 48=432$ Xor gadgets. The total number of gadgets is therefore $960+432=1392$.

Therefore, each PRG from the first class must generate $\alpha_{1}=960$ pseudo-randoms, while each PRG from the 3 other classes must generate $\alpha_{2}=1392$ pseudo-randoms. With the 3 -wise function $G$, in order to generate $\alpha$ pseudo-randoms, each PRG requires $2 \times\lceil\sqrt{\alpha}\rceil$ true randoms for its seed. Therefore, the total number of TRNGs is:

$$
n_{r}=2 \times\left\lceil\sqrt{\alpha_{1}}\right\rceil \times n(n-1) / 2+2 \times\left\lceil\sqrt{\alpha_{2}}\right\rceil \times 3(n-1)
$$

We obtain $n_{r}=642$ for $n=3$ and $n_{r}=1056$ for $n=4$. 